\title{
Electron-Nitrogen Molecule Collisions in High-Temperature Nonequilibrium Air
}

\author{
W.M. Huo* \\ University of Notre Dame, Notre Dame, Indiana \\ and \\ V. McKoy, $\dagger$ M.A.P. Lima, $\ddagger$ and T.L. Gibson $\S$ \\ California Institute of Technology, Pasadena, California
}

\section{Abstract}

$\mathrm{Ab}$ initio calculation of vibrational excitation and de-excitation cross sections of $\mathrm{N}_{2}$ by low-energy resonant electron impact have been carried out. The calculation includes initial target state in $\mathrm{v}=0-12$ and final target state in $\mathrm{v}=0-17$. The calculated cross sections are found to agree with available experimental data to within reported experimental error. Effect of target rotation has been investigated. Comparison of vibrational excitation cross sections at $\mathrm{J}=0,50$, and 150 shows that the major effect of target rotation is to lower the resonance energy. Vibrational excitation and de-excitation rate coefficients are tabulated for electron temperatures of $1100-55,000 \mathrm{~K}$. The present tabulation supersedes an earlier compilation by the Naval Research Laboratory that was derived from experimental data with a factor-of-two error. The tabulated rate coefficients are

Presented as Paper 85-1034 at the AIAA 20th Thermophysics Conference, Williamsburg, VA, June 19-21, 1985. Copyright (c) 1985 by the American Institute of Aeronautics and Astronautics, Inc. All rights reserved.

*Associate Professional Specialist, Radiation Laboratory. Mailing address: NASA Ames Research Center, Moffett Field, CA 94035.

†Professor of Theoretical Chemistry, Department of Chemistry. \$Graduate student. On leave from Instituto de Estudos Avancados, Centro Técnico Aerospacial, Brazil.

§Research fellow, Department of Chemistry; currently with Texas Tech University. 
suited for flowfield modeling of aeroassisted orbital transfer vehicles.

\section{Introduction}

The aeroassisted orbital transfer vehicle (AOTV) is designed to transport payloads between the space station or Space Shuttle in low-altitude equatorial orbits to distant objects in geosynchronous or polar orbits. Since the AOTV trajectories will be largely in the low-density atmosphere, the gas in and immediately behind the shock layer will not be in chemical or thermodynamical equilibrium. As a result of this nonequilibrium, a large radiative heat load is expected. Menees ${ }^{1}$ estimated that more than half of the heat load experienced by the vehicle derives from the nonequilibrium radiative heating. Park $^{2,3}$ has characterized this nonequilibrium regime using a multitemperature model in which the rotational and translational energy modes are equilibrated by a few collisions and climbs rapidly to a temperature of $\approx 50,000 \mathrm{~K}$. On the other hand, it takes much longer for vibrational and electronic energy modes to reach equilibrium. Thus, the electronic and vibrational temperatures remain low, $\leq 15,000 \mathrm{~K}$.

A large chemical and physical data base on radiative intensities, excitation cross sections, chemical reaction rates, etc., are required to model this nonequilibrium flowfield and the associated radiation. 4 Electronmolecule inelastic collision cross sections constitute one part of this data base. There is considerable ionization occurring in the flow and free electrons actually extend in front of the shock wave. Due to its light mass, electron-molecule collisions are $\approx 200$ times more frequent than molecule-molecule collisions. Thus, electronmolecule collisions are effective as an excitation mechanism. In this paper, we present a calculation of $\mathrm{N}_{2}$ vibrational excitation rates by low-energy electron impact. This rate is needed in determining the vibrational temperature as well as the relaxation time of the nonequilibrium gas. ${ }^{5}$

In the 10,000-50,000 $\mathrm{K}$ electron temperature range, the $\mathrm{e}-\mathrm{N}_{2}$ vibrational excitation is dominated by the well known ${ }^{2} \pi_{g}$ resonance, ${ }^{6}$ in which an incident $d$ wave 
electron (angular momentum quantum number $=2$ ) is temporarily trapped inside the molecule. This resonance has been extensively studied, both experimentally and theoretically. ${ }^{6-17}$ However, in all of the experimental measurements, the target is initially in the ground or first excited vibrational level, with rotational temperature being at room temperature. While previous theoretical calculations have included up to vibrational quantum number $\mathrm{v}=8$ in the initial target state, ${ }^{12-14,17}$ molecular rotation has been either neglected or kept at the lowest six rotational levels. Thus, the available experimental and theoretical data are not suited for AOTV modeling. Furthermore, the most systematic measurements of e- $\mathrm{N}_{2}$ vibrational excitation cross sections, carried out by Schulz and co-workers in 1964-1973, provide values "too low by a factor up to two."7 This factor-of-two error has been confirmed by later experiments 9,10 and calculations. ${ }^{12,16,17}$ Unfortunately, the most recent Naval Research Laboratory (NRL) compilation of $\mathrm{N}_{2}$ vibrational excitation cross sections by $\mathrm{Ali}, 18$ as well as the compilation of the vibrational excitation rate coefficients by Slinker and Ali, ${ }^{19}$ are all based on Schulz's data. In the present study, we have calculated e- $\mathrm{N}_{2}$ vibrational excitation and de-excitation cross sections and rate coefficients with the initial $\mathrm{N}_{2}$ vibrational quantum number at $\mathrm{v}=0-12$, and rotational quantum number at $\mathrm{J}=0,50$, and 150. We include all of the final target states, which differ from the initial target state by vibrational quantum numbers of $\pm 1-5$. The tabulated rate coefficients supersede the NRL compilation and are directly applicable in AOTV flowfield calculations.

\section{Computational Method}

The calculation of vibrational excitation and de-excitation rate coefficients involves the following four-step process:

1) Fixed-nuclei e- $\mathrm{N}_{2}$ scattering calculation based on the multichannel Schwinger variational method, ${ }^{20}$ carried out over a range of internuclear distances (1.5-2.6 Bohr, 1 Bohr $\left.=0.529177 \times 10^{-8} \mathrm{~cm}\right)$ and incident electron energies $(0.01-8.2 \mathrm{eV}, 1 \mathrm{eV}=11,605.4 \mathrm{~K})$.

2) Determine entry amplitude $U_{d E}$, width $\Gamma$, and shift $\Delta$ functions from electron scattering calculation. 
3) Using the previously determined $U_{d E}, \Gamma$, and $\Delta$, calculate the vibrational excitation and de-excitation cross sections for $\mathrm{N}_{2}$, with the initial target state at $\mathrm{v}=0-12$ and final target state at $\mathrm{v}=0-17$ and over $\mathrm{a}$ range of incident electron energies of $0.1-8 \mathrm{eV}$.

4) Calculate e- $\mathrm{N}_{2}$ vibrational excitation and de-excitation rate coefficients from the cross sections obtained above.

The details of the calculation will be published elsewhere. 21,22 A brief summary is given below. The fixed-nuclei e- $\mathrm{N}_{2}$ scattering calculation uses the multichannel scattering formulation, based on the Schwinger variational principle, as developed recently by Takatsuka and McKoy. ${ }^{20}$ This method is briefly summarized below. It begins with a projected Lippman-Schwinger equation,

$$
\mathrm{P \Psi}_{\mathrm{m}}^{(+)}=\mathrm{S}_{\mathrm{m}}+\mathrm{G}_{\mathrm{P}}^{(+)} \mathrm{V \Psi}_{\mathrm{m}}^{(+)}
$$

where $\Psi_{m}^{(+)}$is the total $(N+1)$ particle wave function with plane wave and outgoing wave boundary conditions for the mth channel. The projection operator $P$ defines the open-channel space in terms of the eigenfunctions $\Phi_{m}$ of the target Hamiltonian $\mathrm{H}_{\mathrm{N}}$,

$$
P=\sum_{m=1}^{M_{0}}\left|\Phi_{m}\right\rangle\left\langle\Phi_{m}\right|
$$

and

$$
\mathrm{H}_{\mathrm{N}}\left|\Phi_{\mathrm{m}}\right\rangle=\mathrm{E}_{\mathrm{m}}\left|\Phi_{\mathrm{m}}\right\rangle
$$

The open channels are associated with those target states with energy $E_{m}$ less than the total energy of the e + molecule system. In Eq. (1), $S_{m}$ is the free-particle solution of the unperturbed Hamiltonian, $\mathrm{H}_{\mathrm{N}}+\mathrm{T}_{\mathrm{N}+1}$ and is given by

$$
S_{m}=\Phi_{m} e^{i \tilde{k}_{m} \cdot \tilde{r}_{N+1}}
$$

The potential $V$ includes both interactions between the incident and target electrons and between the incident 
electron and the nuclei. The outgoing-wave Green's function $G_{p}^{(+)}$, which is defined only in the open channel space, is given by

$$
G_{P}^{(+)}=-\frac{1}{2 \pi} \sum_{m}^{M}\left|\Phi_{m}>\frac{e^{i k_{m}\left|\tilde{r}_{N+1}-\tilde{r}_{N+1}^{\prime}\right|}}{\left|\tilde{r}_{N+1}-\tilde{r}_{N+1}^{\prime}\right|}<\Phi_{m}\right|
$$

for ${\underset{\psi}{m}}_{++}^{\text {As shown by Takatsuka and McKoy, a complete equation }}$

$\left\{\frac{1}{2}(P V+V P)-V G_{P}^{(+)} V+\frac{1}{N+1}\left[\hat{H}-\frac{N+1}{2}(\hat{P H}+\hat{H P})\right]\right\} \Psi_{m}^{(+)}$

$$
=\mathrm{VS}_{\mathrm{m}}
$$

where $\hat{H}=E+E_{0}-H_{N+1}$ with $E$ the incident electron energy and $E_{0}$ the energy of the initial target state. This equation contains information about the closed electronic channels without defining the closed-channel Green's function, which would necessitate including the target continuum states. Based on Eq. (6), a variational expression for the fixed-nuclei scattering amplitude is

$$
\begin{aligned}
f\left(\tilde{\mathrm{k}}_{\mathrm{n}}, \tilde{\mathrm{k}}_{\mathrm{m}}\right)= & -\frac{1}{2 \pi}\left\langle\mathrm{S}_{\mathrm{n}}|\mathrm{V}| \Psi_{\mathrm{m}}^{(+)}\right\rangle\left\langle\psi_{\mathrm{n}}^{(-)}\right|\left\{\frac{1}{2}(\mathrm{PV}+\mathrm{VP})\right. \\
& -\mathrm{VG}_{\mathrm{p}}^{(+)} \mathrm{V}+\frac{1}{\mathrm{~N}+1}\left[\hat{\mathrm{H}}-\frac{\mathrm{N}+1}{2}(\mathrm{PH}\right. \\
& +\hat{\mathrm{HP}})]\}\left|\psi_{\mathrm{m}}^{(+)}\right\rangle^{-1}\left\langle\psi_{\mathrm{n}}^{(-)}|\mathrm{V}| \mathrm{S}_{\mathrm{m}}\right\rangle
\end{aligned}
$$

In our calculation, $\Psi_{m}^{(+)}$is expanded in a basis of $(\mathrm{N}+1)$-particle determinants. These determinants are constructed using the natural orbitals from a configuration interaction calculation of the ${ }^{2} \pi_{g}$ state of the $\mathrm{N}_{2}^{-}$ ion. ${ }^{21}$ The natural orbitals are in turn expanded in a Gaussian basis set with $11 s 8 p 3 d$ basis functions on each nucleus. The basis set was obtained by uncontracting 
the $9 s 5 p 2 d / 5 s 3 p 2 d$ basis of Langhoff et al. ${ }^{23}$ and adding diffuse $s, p$, and $d$ functions to provide a better description of the continuum electron. The $9 s 5 p 2 d / 5 s 3 p 2 d$ basis set has been used by Langhoff et al. to calculate the static polarizability with good results. ${ }^{23}$ Our calculation used the static exchange + polarization level of approximation, ${ }^{24}$ which has been shown to provide reliable widths for the $2 \pi_{\mathrm{g}}$ resonance in $\mathrm{N}_{2} \cdot 25$ It is found that the use of negative ion natural orbitals expedites the convergence of our calculation significantly.

To deduce the width and shift functions from the fixed-nuclei electron scattering calculation, we rewrite the scattering amplitude for the $\ell=2$ partial wave in terms of the $K$ matrix,

$$
\mathrm{f}_{\ell=2}=\frac{\mathrm{K}}{2(1-i \mathrm{k})}
$$

The $\mathrm{K}$ matrix element at a given internuclear distance $R$ and incident electron energy $E$ is related to the fixed-nuclei width $\Gamma(E, R)$ and shift $\Delta(E, R)$ functions by

$$
K(E, R)=-\frac{\Gamma(E, R) / 2}{E-\varepsilon_{d}(R)-\Delta(E, R)}
$$

where $\varepsilon_{d}$ is the potential difference between the target and negative ion vibration and the values deduced by Berman et al. ${ }^{17}$ are used in our calculation. In fitting $\Gamma(E, R)$, we choose the same functional form as used by Berman et al.,

$$
\Gamma(E, R)=2 \pi E^{\ell+1 / 2}\left|\sum_{j=1}^{3} A_{j} e^{-C_{j}\left(R-R_{e}\right)} e^{-B_{j} E}\right|^{2}
$$

with $R_{e}$ the equilibrium internuclear distance of the target (2.07435 Bohr). The fixed-nuclei shift function is given by 


$$
\Delta(E, R)=\frac{1}{2 \pi} p \int d E^{\prime} \frac{\Gamma\left(E^{\prime}, R\right)}{E-E^{\prime}}
$$

where $p$ denotes Cauchy's principle part of the integral. When the functional form in Eq. (10) is used for $\Gamma(E, R)$, the entry amplitude $U_{d E}$ is given simply by

$$
U_{d E}(E, R)=E^{(\ell+0.5) / 2} \sum_{j=1}^{3} A_{j} e^{-C_{j}\left(R-R_{e}\right)} e^{-B_{j} E}
$$

Our final set of parameters $A_{j}, B_{j}$, and $C_{j}$, are chosen to give the best fit to both our ab initio $K$ matrix and the $a b$ initio width function of Hazi.

The calculation of vibrational excitation cross section follows a procedure based on the Feshbach projection operator formalism ${ }^{26}$ and has been described by Berman et al. ${ }^{17}$ Since the vibrational excitation of $\mathrm{N}_{2}$ is dominated by a single partial wave, the excitation cross section from level $v_{i}$ to $v_{f}$ is given by

$$
\begin{aligned}
\sigma_{i f}= & (2 \pi)^{3}\left(n_{i} / E\right) \mid\left\langle v_{f}\right| U_{E_{f} d}\left[E-T_{v i b}\right. \\
& \left.-v_{o p t}\left(E-H_{v i b}\right)\right]\left.^{-1} U_{E_{i} d}\left|v_{i}\right\rangle\right|^{2}
\end{aligned}
$$

where $n_{i}$ is the spatial degeneracy of the negative ion wave function, $\mathrm{T}_{\mathrm{vib}}$ is the kinetic energy operator for vibrational motion, and $V_{\text {opt }}$ is the optical potential

$V_{o p t}\left(E-H_{v i b}\right)=V_{o}+\varepsilon_{d}(R)+\Delta\left(E-H_{v i b}\right)-\frac{i}{2} \Gamma\left(E-H_{v i b}\right)$

$\mathrm{H}_{\mathrm{vib}}$ is the target vibrational Hamiltonian,

$$
\begin{aligned}
& H_{v i b}=T_{v i b}+V_{0} \\
& V_{0}=V_{R K R}+\frac{J(J+1) h}{8 \pi^{2} c \mu R^{2}}
\end{aligned}
$$




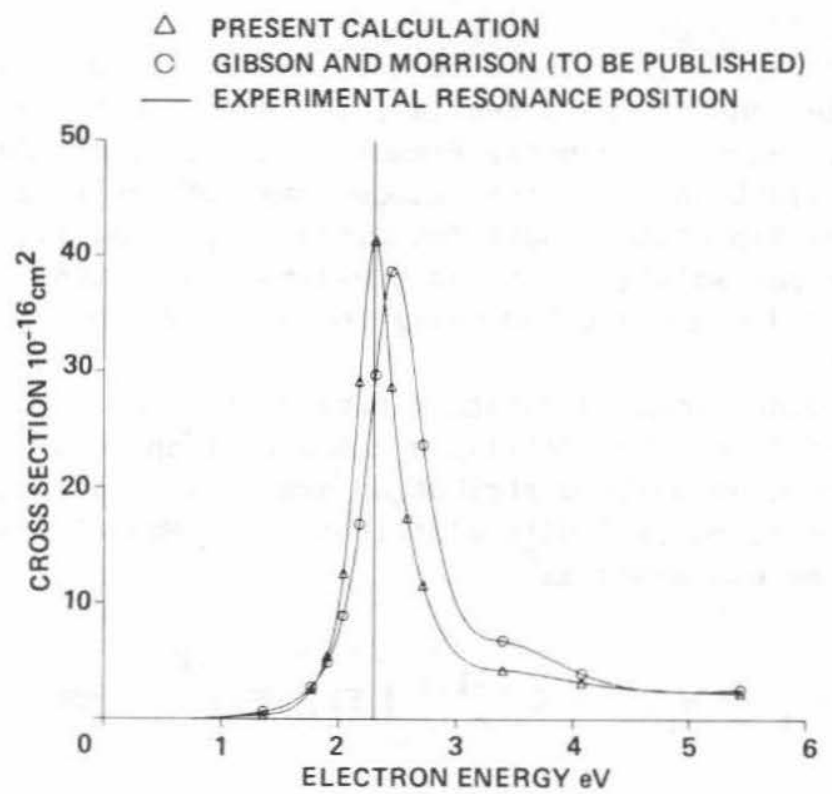

Fig. 1 e- $\mathrm{N}_{2}$ fixed-nuclei cross section at $R=2.068$ Bohr.

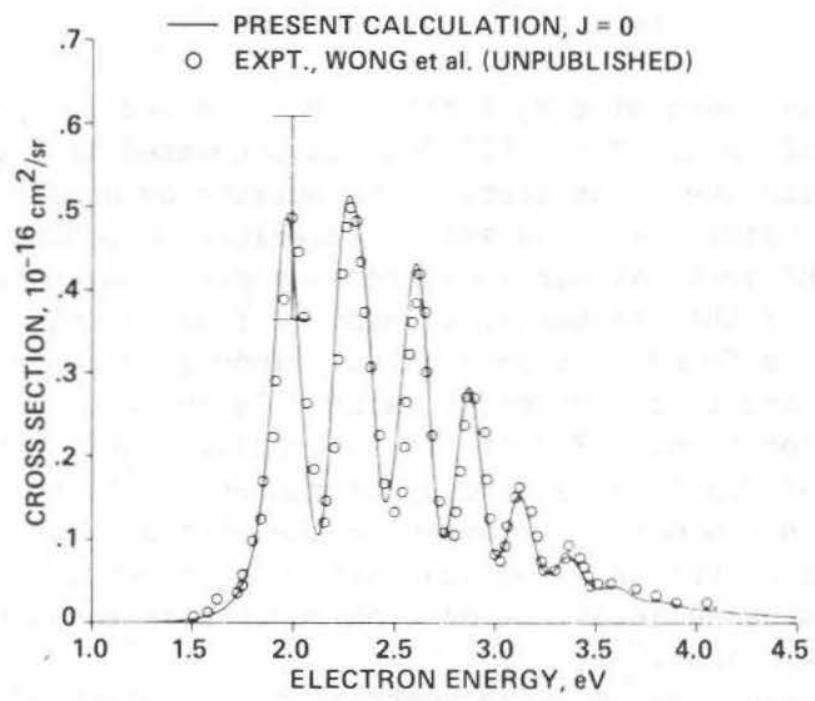

Fig. 2 e-N $2 \quad v=0-1$ cross section at $90 \mathrm{deg}$. 
The RKR potential for the target is deduced from experimental spectroscopic constants. ${ }^{27}$ We have extended this potential to large $R$ using the ab initio data of Bauschlicher. ${ }^{28}$ The second term in Eq. (16), corresponding to the centrifugal potential, accounts for the target rotation. Here $h$ denotes Planck's constant, $c$ the speed of light, and $\mu$ the reduced mass of the molecule. The vibrational wave functions $\left|v_{i}\right\rangle$ and $\left|v_{f}\right\rangle$ are numerical solutions of the one-dimensional Schrödinger equation and are evaluated using the Numerov-Cooley technique.

The vibrational excitation rate coefficient $k_{\text {if }}$ can be evaluated once the collision cross section $\sigma_{i f}$ and the electron velocity distribution are known. Assuming that the electron velocity distribution is Maxwellian, $\mathrm{k}_{\text {if }}$ can be expressed as ${ }^{5}$

$$
k_{i f}=\left\langle\sigma_{i f} v\right\rangle=C_{o} T_{e}^{-1.5} \int E \sigma_{i f}(E) e^{-E / T} e d E
$$

where $C_{0}$ is $6.6971 \times 10^{-7}, E$ the electron energy, and $\mathrm{T} e$ the electron temperature in units of $\mathrm{eV}$. The cross section is in units of $\mathrm{cm}^{2}$ and $\mathrm{k}_{\text {if }}$ in $\mathrm{cm}^{3} / \mathrm{s}$.

\section{Results and Discussions}

The fixed-nuclei e- $\mathrm{N}_{2}$ scattering cross section from our calculation at $R=2.068$ Bohr is presented in Fig. 1, together with the cross sections calculated by Gibson and Morrison ${ }^{29}$ using the nonadiabatic polarization potential method. The vertical bar indicates the electron energy at the center of the resonance, as deduced from experiment. ${ }^{7}$ It is found that both calculations give resonance energies close to experimental value. In addition, our resonance position, $2.313 \mathrm{eV}$, is in good agreement with the value of $2.316 \mathrm{eV}$ deduced by Berman et al. ${ }^{17}$ from Hazi's ab initio data. ${ }^{16}$ Comparing the shapes of the two theoretical curves, we find qualitative agreement, with the present calculation giving a narrower peak and a $6 \%$ higher peak height.

For low-energy $d$ wave scattering, the width of the resonance is related to the resonance energy 30 by the 
$\mathrm{e}-\mathrm{N}_{2} \mathrm{v}=0$ TO 2 CROSS SECTION AT $90^{\circ}$

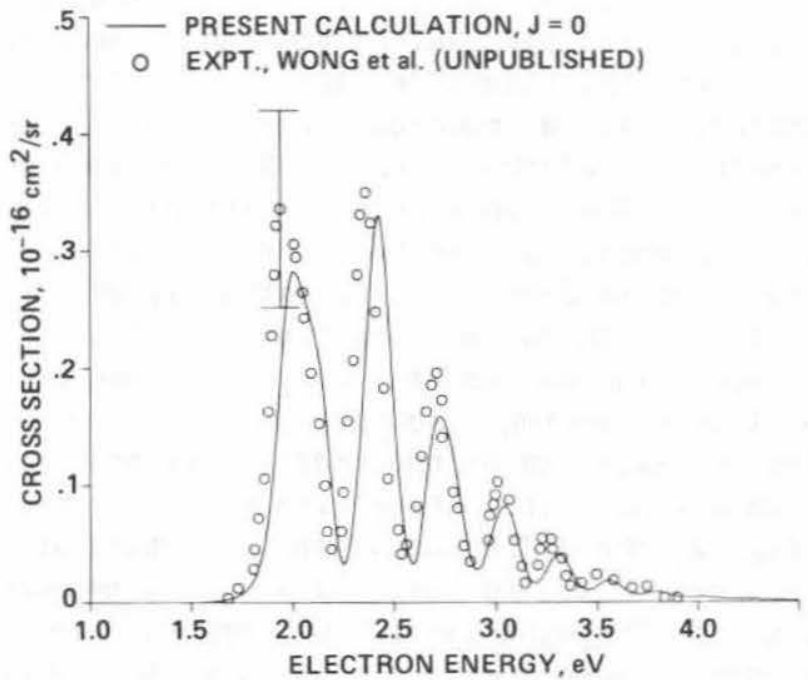

Fig. 3 e-N $2 \quad v=0-2$ cross section at $90 \mathrm{deg}$.

\section{$e-N_{2} v=1$ TO 2 CROSS SECTION AT $90^{\circ}$}

- PRESENT CALCULATION, $\mathrm{J}=0$

O EXPT., WONG et al. (UNPUBLISHED)

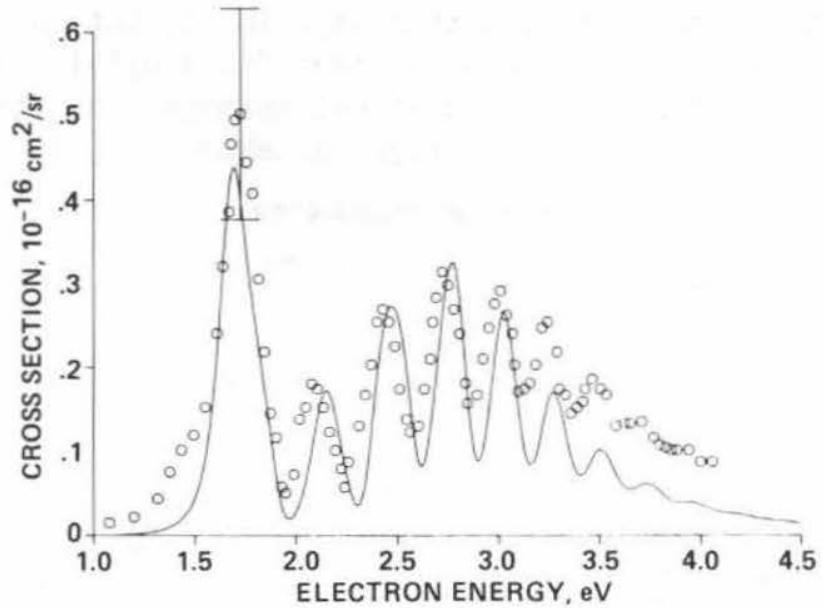

Fig. 4 e-N $2 \quad v=1-2$ cross section at 90 deg. 
factor $E^{2.5}$. This relation is found to hold well in the $\mathrm{R}$ dependence of the calculated width. The position of the calculated resonance shifts to higher energies with decreasing $R$, and the resonance width is found to increase sharply. As $R$ becomes larger than $R_{e}$, both resonance energy and width decrease. No resonance is found at $R>2.40$ Bohr, in agreement with Hazi's calculation. This is probably a result of the fact the resonance energy is so close to zero that the extremely weak peaks are not discernible in our calculation. Details of the fixed-nuclei calculation, as well as the fitting of $\Gamma, \Delta$, and $U_{d E}$, will be presented elsewhere. ${ }^{21,22}$

To test the validity of the theoretical cross sections, we compare our calculation with recent experimental data. In Fig. 2, the differential cross section at $90 \mathrm{deg}$ for $\mathrm{v}=0-1$ excitation is compared with the measurement of Wong et al. ${ }^{10}$ The experimental measurement was carried out at room temperature and $\mathrm{J}=0$ was used in our calculation. The relative accuracy of the measurement at different energies is reported to be about $\pm 10 \%$ and the absolute experimental cross section has an error of $\pm 25 \% .13$ The experimental uncertainty is indicated by the error bar at one peak. Theory and experiment are found to be in agreement in the entire spectrum.

Figures 3 and 4 compare the 90 deg differential cross sections for $\mathrm{v}=0-2$ and $1-2$ with the measurement of Wong et al. ${ }^{10}$ For $\mathrm{v}=0-2$, we find that the theoretical cross sections are $16 \%$ less than experiment, but the difference between theory and experiment falls within experimental error. Also, the theoretical resonance position appears to be shifted to the right of experiment by

e- $\mathrm{N}_{2} v=0$ TO 1 TOTAL CROSS SECTION

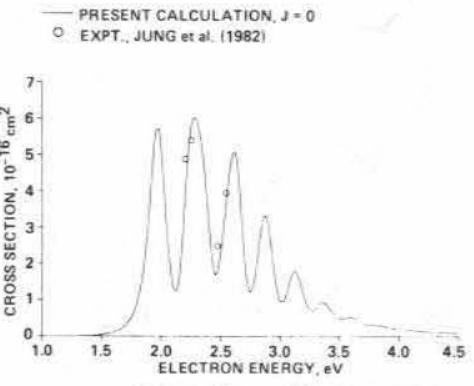

Fig. 5 e- $\mathrm{N}_{2} \quad \mathrm{v}=0-1$

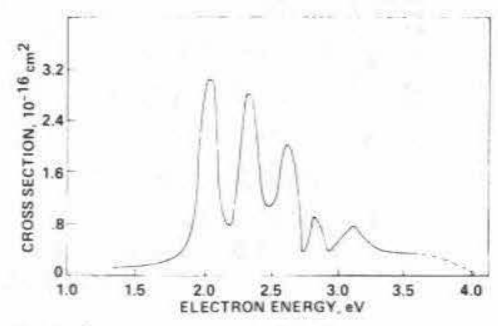

total cross section. 
$\approx 0.1 \mathrm{eV}$. Similar results are found for the $v=1-2$ excitation in Fig. 4.

The total vibrational excitation cross section is a quantity directly useful to AOTV modeling. On the left hand side of Fig. 5, we compare our calculation for $v=0-1$ with the recent measurement of Jung et al. 9

While their measurement provides only four data points, they all fall on the theoretical curve. On the right hand side of Fig. 5, we reproduced Schultz's experimental spectrum for this transition. ${ }^{31}$ Comparing the peaks heights of the two spectra, Schulz's measurement is indeed found to be too small by approximately a factor of two. There is also considerable difference in the shape of the two spectra.

To illustrate the effect of molecular rotation in e- $\mathrm{N}_{2}$ vibrational excitation, we plot in Fig. 6 the $\mathrm{v}=0-1$ cross section calculated with $\mathrm{J}=0$ and 50 . It is seen that the major effect of introducing high rotational temperature $(\approx 7400 \mathrm{~K})$ is to shift the resonance peak lower by $0.1 \mathrm{eV}$. The peak height for the $\mathrm{J}=50$

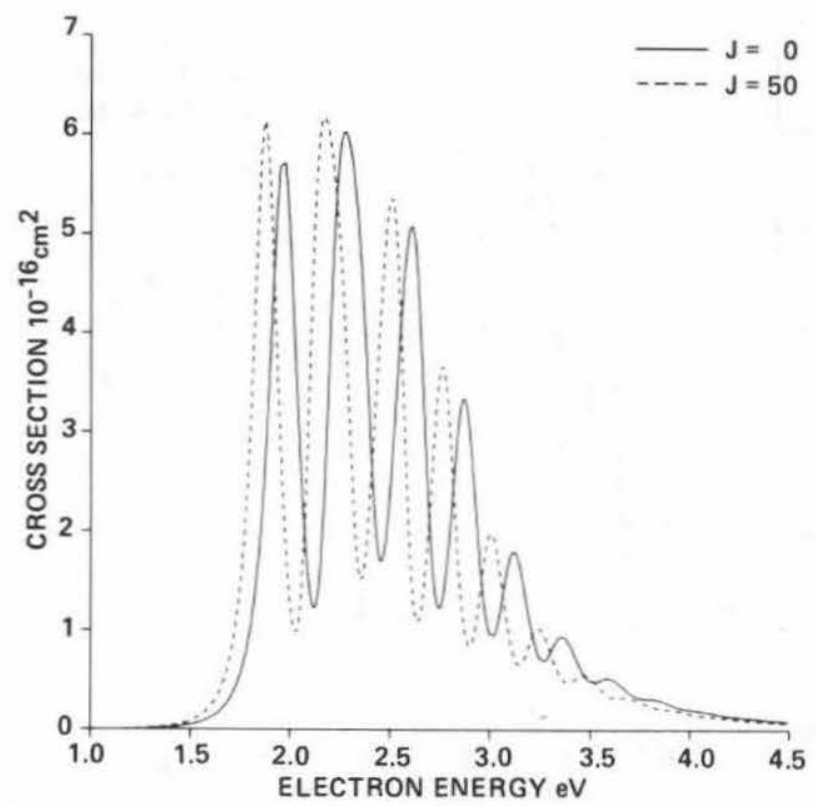

Fig. 6 e-N $2 \quad v=0-1$ total cross section for $J=0$ and 50 . 


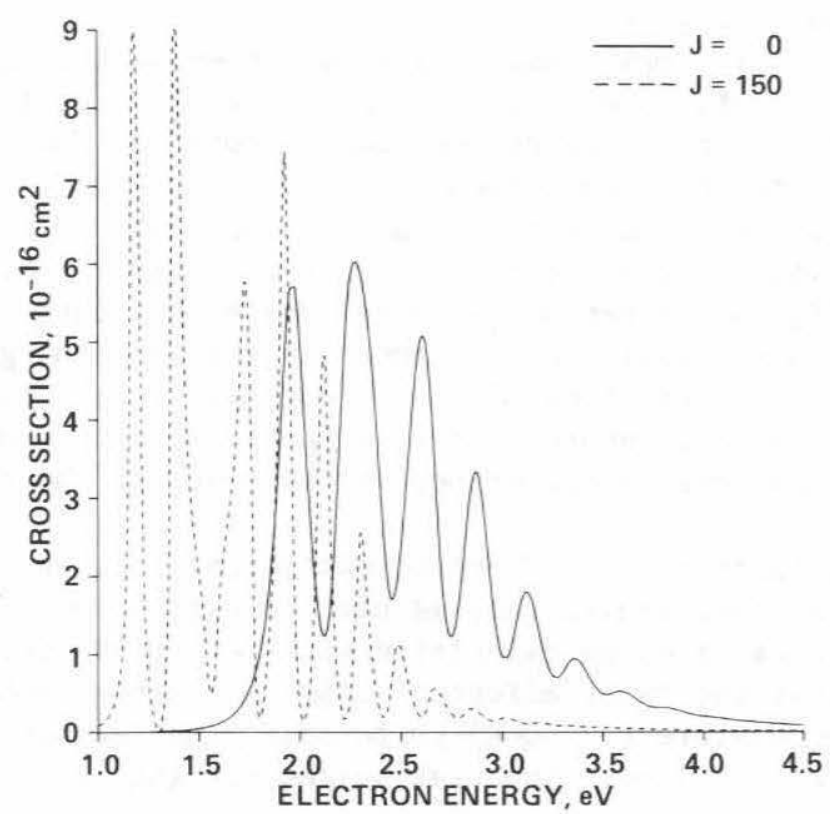

Fig. 7 e-N $\mathrm{N}_{2} \quad \mathrm{v}=0-1$ total cross section for $J=0$ and 150 .

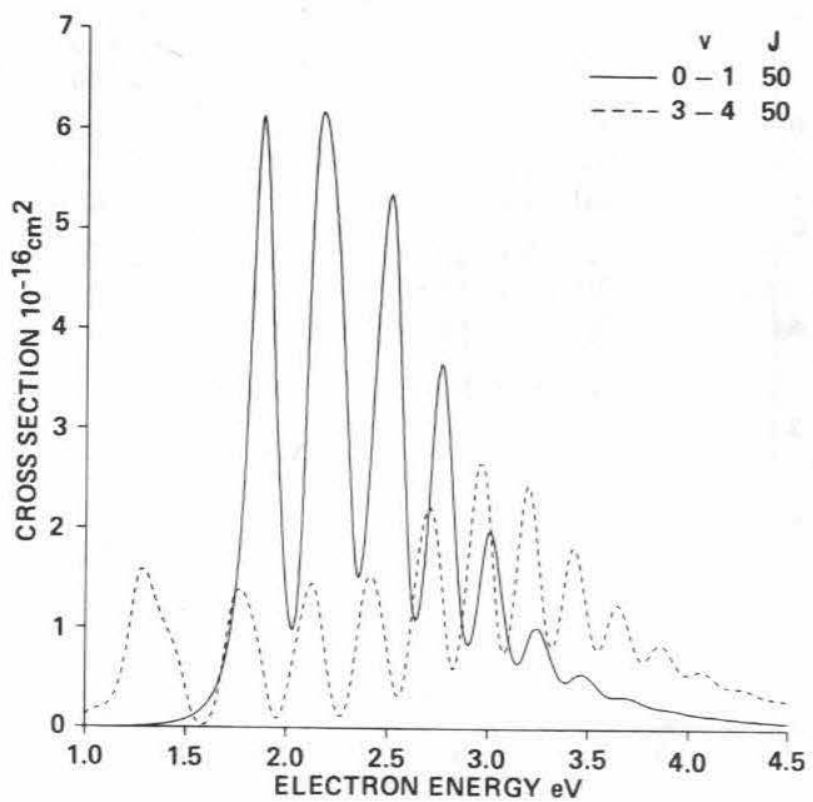

Fig. 8 e- $\mathrm{N}_{2} \quad \mathrm{v}=0-1$ and $3-4$ excitation cross sections. 


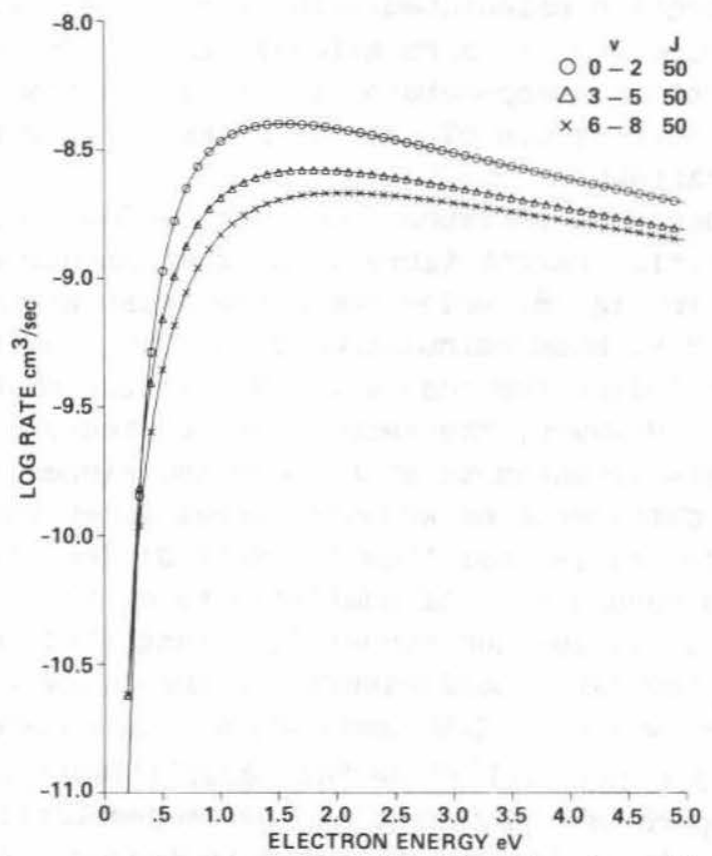

Fig. $9 \quad e-N_{2} \quad v=0-2,3-5$, and $6-8$ vibrational excitation rates.

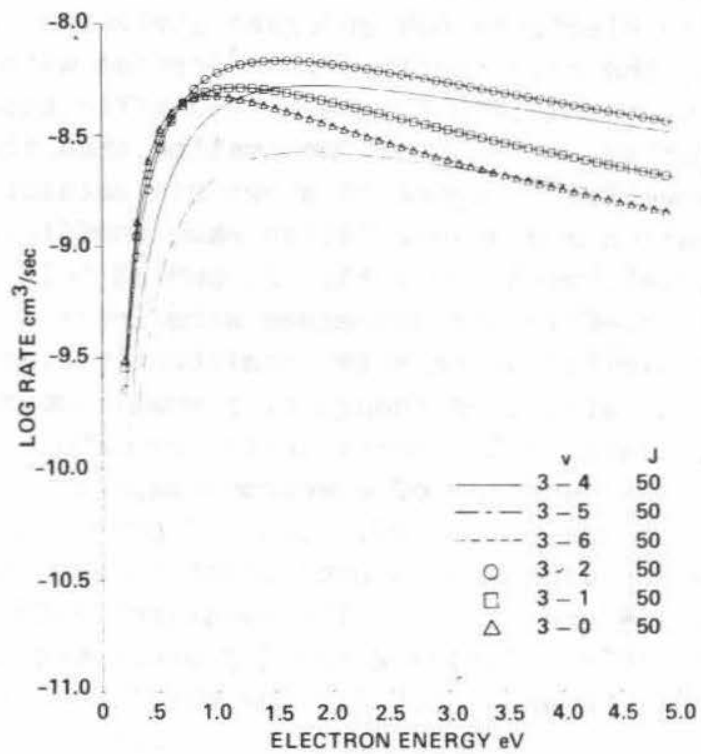

Fig. 10 e-N 2 vibrational excitation and de-excitation rates out of $v=3$. 
spectrum is also slightly higher. Fig. 7 compares the same cross section calculated with $J=0$ and 150 , the latter corresponding to a rotational temperature of $66,000 \mathrm{~K}$. At this temperature, the first resonance peak is shifted lower by $0.8 \mathrm{eV}$ and the peaks are significantly higher and narrower.

The dependence of vibrational excitation cross section with initial target vibrational quantum number is illustrated in Fig. 8, which shows the cross section for $v=0-1$ and $3-4$, both calculated at $J=50$. We find the maximum peak height for the $v=3-4$ excitation to be $43 \%$ of $v=0-1$. However, the resonances for the $v=3-4$ excitation are extended to both lower and higher energies, so that the difference between the areas under the resonance spectra is less than the peak difference.

The dependence of rate coefficients on the initial state vibrational quantum number is illustrated in Fig. 9, which shows the rate coefficients for three $\Delta v=2$ transitions, for $\mathrm{v}=0-2,3-5$, and $6-8$, all calculated with $J=50$. We see that all three rate coefficients are strongly temperature dependent at low temperatures and increase sharply at the onset of the resonance. At the high-temperature end, the rate coefficients decrease slowly, reflecting the fact that an increasing portion of the Maxwellian electrons has energies above the resonance. Also, the rate coefficients decrease with increasing $v$. At $15,000 \mathrm{~K}$, the rate coefficient for the 6-8 transition is a factor of two smaller than the $v=0-2$ transition. Figure 10 shows six sets of vibrational excitation and de-excitation rate coefficients out of $v=3$ level, with $\Delta v= \pm 1, \pm 2$, and \pm 3 . It is seen that the rate coefficient decreases with increasing $\Delta \mathrm{v}$. Also, the de-excitation rate is consistently higher than the excitation rate, even though by a small amount.

The calculated rate coefficients are tabulated in Tables $1-27^{\circ}$ as a function of electron temperatures at $0.1-5.0 \mathrm{eV}$ in steps of $0.1 \mathrm{eV}$. Table 1 presents the rate coefficients for vibrational excitation out of $v=0$, with $\Delta v$ of $1-5$ and $J=0$. The notation E-08 in the table denotes $10^{-8}$. Tables 2 and 3 present the same quantities for $J=50$ and 150 . All the rate coefficients

${ }^{\star *}$ Tables 1-27 appear on pp. 168-193. 
presented in Tables 4-27 are calculated using $\mathrm{J}=50$. Tables 4-15 present the vibrational excitation rate coefficients out of initial target states $v=0-12$, with $\Delta \mathrm{v}=1-5$. Tables 16-27 present the corresponding de-excitation rate coefficients, with $\Delta v=-1$ to -5 . A comparison of Tables $1-3$ shows that the excitation rate coefficient increases with $\mathrm{J}$ at low electron temperatures and decreases with $J$ at high temperatures. The transition between the two regimes occurs between 1.0 and $1.6 \mathrm{eV}$, depending on the particular vibrational excitation considered. Similar $J$ dependence has been found for the rate coefficients of other vibrational transitions. The tabulated rate coefficients can be used directly to determine vibrational temperature and relaxation times.

\section{Summary}

In the present study, we have determined $e-\mathrm{N}_{2}$ vibrational excitation and de-excitation cross sections and rate coefficients using ab initio methods. The effect of high vibrational and rotational temperatures are investigated. It is found that the rate coefficients decrease slowly with increasing vibrational temperature, but increase with rotational temperature at low electron temperatures. The latter is mainly due to the fact that the high rotational temperature of the target state lowers the resonance energy of the well known e- $\mathrm{N}_{2}{ }^{2} \mathrm{I}_{\mathrm{g}}$ resonance. The rate coefficients are also found to decrease sharply with increasing $\Delta v$, dropping by approximately a factor of two when $\Delta v$ is increased by one.

Our calculated cross sections are found to be in good agreement with the available experimental data. Thus, the tabulated rate coefficients can be used in AOTV flowfield modeling with confidence.

\section{Acknowledgment}

We would like to thank Dr. A. U. Hazi for supplying us with his unpublished data. The research of W. M. Huo is supported by NASA under NASA Ames Cooperative Agreement NCC 2-147. The research of V. McKoy, M. A. P. Lima, and T. L. Gibson is supported by NASA under NASA Ames Cooperative Agreement NCC 2-319. 
Table 1 e- $\mathrm{N}_{2}$ vibrational excitation rate coefficients, $\mathrm{cm}^{3} / \mathrm{s}$ $\left(v_{i}=0, J=0\right)$

\begin{tabular}{|c|c|c|c|c|c|}
\hline $\mathrm{T}, \mathrm{eV}$ & $v_{f}=1$ & 2 & 3 & 4 & 5 \\
\hline 0.1 & $0.1046 \mathrm{E}-13$ & $0.1089 \mathrm{E}-14$ & $0.2927 \mathrm{E}-15$ & $0.8031 E-16$ & $0.1737 \mathrm{E}-16$ \\
\hline 0.2 & $0.1476 \mathrm{E}-10$ & $0.6318 \mathrm{E}-11$ & $0.3078 \mathrm{E}-11$ & $0.1357 \mathrm{E}-11$ & \\
\hline 0.3 & $0.2300 \mathrm{E}-09$ & $0.1095 \mathrm{E}-09$ & $0.5974 E-10$ & $0.3048 \mathrm{E}-10$ & $0.1349 E-10$ \\
\hline 0.4 & $0.8736 \mathrm{E}-09$ & $0.4262 E-09$ & $0.2424 \mathrm{E}-09$ & $0.1321 \mathrm{E}-09$ & $0.6375 \mathrm{E}-10$ \\
\hline 0.5 & $0.1869 \mathrm{E}-08$ & $0.9166 \mathrm{E}-09$ & $0.5313 \varepsilon-09$ & $0.3001 \mathrm{E}-09$ & $0.1522 E-09$ \\
\hline 0.6 & $0.3005 E-08$ & $0.1472 E-08$ & $0.8615 \mathrm{E}-09$ & $0.4974 E-09$ & $0.2605 \mathrm{E}-09$ \\
\hline 0.7 & $0.4110 \mathrm{E}-08$ & $0.2007 \varepsilon-08$ & $0.1181 \mathrm{E}-08$ & $0.6917 \mathrm{E}-09$ & $0.3704 E-09$ \\
\hline 0.8 & $0.5091 \mathrm{E}-08$ & $0.2478 \mathrm{E}-08$ & $0.1461 \mathrm{E}-08$ & $0.8648 \mathrm{E}-09$ & $0.4707 \mathrm{E}-09$ \\
\hline 0.9 & $0.5913 E-08$ & $0.2866 \mathrm{E}-08$ & $0.1693 \mathrm{E}-08$ & $0.1009 \mathrm{E}-08$ & $0.5562 E-09$ \\
\hline 1.0 & $0.6573 E-08$ & $0.3174 \mathrm{E}-08$ & $0.1876 \mathrm{E}-08$ & $0.1125 E-08$ & $0.6258 \mathrm{E}-09$ \\
\hline 1.1 & $0.7083 E-08$ & $0.3408 \mathrm{E}-08$ & $0.2015 \mathrm{E}-08$ & $0.1214 \mathrm{E}-08$ & $0.6803 \mathrm{E}-09$ \\
\hline 1.2 & $0.7463 E-08$ & $0.3579 \mathrm{E}-08$ & $0.2115 \mathrm{E}-08$ & $0.1279 \mathrm{E}-08$ & $0.7215 \mathrm{E}-09$ \\
\hline 1.3 & $0.7733 \mathrm{E}-08$ & $0.3697 \mathrm{E}-08$ & $0.2185 E-08$ & $0.1325 E-08$ & $0.7513 E-09$ \\
\hline 1.4 & $0.7914 \mathrm{E}-08$ & $0.3773 E-08$ & $0.2229 \mathrm{E}-08$ & $0.1354 \mathrm{E}-08$ & $0.7716 \mathrm{E}-09$ \\
\hline 1.5 & $0.8021 E-08$ & $0.3814 \mathrm{E}-08$ & $0.2252 E-08$ & $0.1371 \mathrm{E}-08$ & $0.7843 E-09$ \\
\hline 1.6 & $0.8069 \mathrm{E}-08$ & $0.3827 \mathrm{E}-08$ & $0.2259 \mathrm{E}-08$ & $0.1378 \mathrm{E}-08$ & $0.7907 \mathrm{E}-09$ \\
\hline 1.7 & $0.8070 \mathrm{E}-08$ & $0.3819 \varepsilon-08$ & 0.225 & $0.1376 \mathrm{E}-08$ & $0.7921 \mathrm{E}-09$ \\
\hline 1.8 & $0.8034 \mathrm{E}-08$ & $0.3794 E-08$ & $0.2237 E-08$ & $0.1368 \mathrm{E}-08$ & $0.7895 \mathrm{E}-09$ \\
\hline 1.9 & $0.7969 \mathrm{E}-08$ & 0.3756 & $0.2214 E-08$ & $0.1356 \mathrm{E}-08$ & $0.7839 \mathrm{E}-09$ \\
\hline 2.0 & $0.7881 \mathrm{E}-08$ & $0.3708 \mathrm{E}-08$ & $0.2185 E-08$ & $0.1339 \mathrm{E}-08$ & $0.7758 \mathrm{E}-09$ \\
\hline 2.1 & $0.7776 \mathrm{E}-08$ & $0.3652 \mathrm{E}-08$ & $0.2151 \mathrm{E}-08$ & $0.1319 \mathrm{E}-08$ & $0.7659 \mathrm{E}-09$ \\
\hline 2.2 & $0.7658 \mathrm{E}-08$ & $0.3591 \mathrm{E}-08$ & $0.2114 E-08$ & $0.1298 \mathrm{E}-08$ & $0.7546 \mathrm{E}-09$ \\
\hline 2.3 & $0.7531 \mathrm{E}-08$ & 0.352 & $0.2075 E-08$ & $0.1275 E-08$ & $0.7422 E-09$ \\
\hline 2.4 & $0.7396 \mathrm{E}-08$ & $0.3458 \mathrm{E}-08$ & $0.2034 \mathrm{E}-08$ & $0.1250 \mathrm{E}-08$ & $0.7291 \mathrm{E}-09$ \\
\hline 2.5 & $0.7257 \mathrm{E}-08$ & $0.3388 \mathrm{E}-08$ & $0.1993 E-08$ & $0.1225 \mathrm{E}-08$ & $0.7155 \mathrm{E}-09$ \\
\hline 2.6 & $0.7115 \mathrm{E}-08$ & $0.3318 E-08$ & $0.1951 \mathrm{E}-08$ & $0.1200 \mathrm{E}-08$ & $0.7015 \mathrm{E}-09$ \\
\hline 2.7 & $0.6972 \mathrm{E}-08$ & $0.3247 E-08$ & $0.1908 \mathrm{E}-08$ & $0.1175 E-08$ & $0.6874 E-09$ \\
\hline 2.8 & $0.6828 \mathrm{E}-08$ & 0.3177 E-08 & $0.1866 \mathrm{E}-08$ & $0.1149 \mathrm{E}-08$ & $0.6732 E-09$ \\
\hline 2.9 & $0.6685 E-08$ & 0.3107 & $0.1825 \mathrm{E}-08$ & 0.11 & 0.65 \\
\hline 3.0 & $0.6543 E-08$ & $0.3038 \mathrm{E}-08$ & $0.1784 E-08$ & $0.1099 \mathrm{E}-08$ & $0.6450 \mathrm{E}-09$ \\
\hline 3.1 & $0.6403 E-08$ & $0.2970 \mathrm{E}-08$ & $0.1743 \mathrm{E}-08$ & $0.1075 E-08$ & $0.6311 \mathrm{E}-09$ \\
\hline 3.2 & $0.6265 E-08$ & 0.2 & 0.1 & $0.10^{5}$ & 0.61 \\
\hline 3.3 & $0.6130 \mathrm{E}-08$ & $0.2839 \mathrm{E}-08$ & $0.1665 \mathrm{E}-08$ & $0.1027 \mathrm{E}-08$ & $0.6040 \mathrm{E}-09$ \\
\hline 3.4 & 0.5 & 0.2 & 0.162 & 0.10 & 0.59 \\
\hline 3.5 & $0.5868 \mathrm{E}-08$ & $0.2713 E-08$ & $0.1590 \mathrm{E}-08$ & 0.981 & $0.5780 \mathrm{E}-09$ \\
\hline 3.6 & 0.57 & $0.2652 \mathrm{E}-08$ & $0.1555 \mathrm{E}-08$ & $0.9598 \mathrm{E}-09$ & $0.5654 \mathrm{E}-09$ \\
\hline 3.7 & 0.5 & 0.2 & 0.15 & 0.93 & $0.5532 E-09$ \\
\hline 3.8 & $0.5498 \mathrm{E}-08$ & $E-08$ & $0.1486 \mathrm{E}-08$ & $3 E-09$ & $E-09$ \\
\hline 3.9 & 0.5 & & & & $6 E-09$ \\
\hline 4.0 & $0.5267 \mathrm{E}-08$ & 0.24 & 0.14 & $0.8781 \mathrm{E}-09$ & $3 E-09$ \\
\hline 4.1 & $0.5156 \mathrm{E}-08$ & $0.2374 E-08$ & $0.1390 \mathrm{E}-08$ & $0.8591 \mathrm{E}-09$ & $0.5073 E-09$ \\
\hline 4.2 & 0.5 & - 232320 & $0.1360 \mathrm{E}-\mathrm{C}$ & $0.8406 \mathrm{E}-09$ & $0.4966 \mathrm{E}-09$ \\
\hline 4.3 & $4 E-08$ & 0.227 & 0.13 & $0.8228 \mathrm{E}-09$ & $0.4862 \mathrm{E}-09$ \\
\hline 4.4 & $0.4843 E-08$ & $0.2226 \mathrm{E}-08$ & $0.1303 \mathrm{E}-08$ & $0.8054 \mathrm{E}-09$ & $0.4762 E-09$ \\
\hline 4.5 & $0.4744 \mathrm{E}-08$ & $0.2180 E-08$ & $0.1275 \mathrm{E}-$ & $0.7886 \mathrm{E}-09$ & $0.4664 E-09$ \\
\hline 4.6 & $0.4648 \mathrm{E}-08$ & $0.2135 E-08$ & $0.1249 \mathrm{E}-08$ & $0.7722 E-09$ & $0.4569 \mathrm{E}-09$ \\
\hline 4.7 & $0.4555 \mathrm{E}-08$ & $0.2091 E-08$ & & $0.7564 \mathrm{E}-09$ & $0.4476 \mathrm{E}-09$ \\
\hline 4.8 & $0.4465 E-08$ & 0.2 & 0.11 & $0.7410 E-09$ & $0.4387 \varepsilon-09$ \\
\hline 4.9 & 0.43 & $0.2007 \mathrm{E}-$ & $0.1174 \mathrm{E}$ & $0.7261 \mathrm{E}-09$ & $0.4300 \mathrm{E}-09$ \\
\hline 5.0 & $0.4292 E-08$ & $0.1967 \mathrm{E}-08$ & $0.1150 \mathrm{E}-08$ & $0.7116 \mathrm{E}-09$ & $0.4215 \mathrm{E}-09$ \\
\hline
\end{tabular}


Table 2 e- $\mathrm{N}_{2}$ vibrational excitation rate coefficients, $\mathrm{cm}^{3} / \mathrm{s}$ $\left(v_{j}=0, J=50\right)$

\begin{tabular}{|c|c|c|c|c|c|}
\hline $\mathrm{T}, \mathrm{eV}$ & $v_{f}=1$ & 2 & 3 & 4 & 5 \\
\hline 0.1 & $0.1637 \mathrm{E}-13$ & $0.2269 \mathrm{E}-14$ & $0.6790 \mathrm{E}-15$ & $0.1921 E-15$ & $0.4216 \mathrm{E}-16$ \\
\hline 0.2 & $0.2071 \mathrm{E}-10$ & $0.9354 \mathrm{E}-11$ & $0.4686 \mathrm{E}-11$ & $0.2098 \mathrm{E}-11$ & $0.7823 E-12$ \\
\hline 0.3 & $0.2861 \mathrm{E}-09$ & $0.1416 \mathrm{E}-09$ & $0.7886 \mathrm{E}-10$ & $0.4067 \mathrm{E}-10$ & $0.1810 \mathrm{E}-10$ \\
\hline 0.4 & $0.1019 \mathrm{E}-08$ & $0.5151 \mathrm{E}-09$ & $0.29828-09$ & $0.1639 \mathrm{E}-09$ & $0.7939 \mathrm{E}-10$ \\
\hline 0.5 & $0.2097 \mathrm{E}-08$ & $0.1064 E-08$ & $0.6267 \varepsilon-09$ & $0.3565 \mathrm{E}-09$ & $0.1814 E-09$ \\
\hline 0.6 & $0.3282 E-08$ & $0.1663 E-08$ & $0.9882 E-09$ & $0.5742 E-09$ & $0.3014 E-09$ \\
\hline 0.7 & $0.4404 \mathrm{E}-08$ & $0.2224 \mathrm{E}-08$ & $0.1328 \mathrm{E}-08$ & $0.7825 \mathrm{E}-09$ & $0.4197 \varepsilon-09$ \\
\hline 0.8 & $0.5378 \mathrm{E}-08$ & $0.2705 \varepsilon-08$ & $0.1619 E-08$ & $0.9636 \mathrm{E}-09$ & $0.5250 \mathrm{E}-09$ \\
\hline 0.9 & $0.6176 \mathrm{E}-08$ & $0.3094 \mathrm{E}-08$ & $0.1855 \mathrm{E}-08$ & $0.1112 E-08$ & $0.6130 E-09$ \\
\hline 1.0 & $0.6802 E-08$ & $0.3395 \varepsilon-08$ & $0.2036 \mathrm{E}-08$ & $0.1227 \mathrm{E}-08$ & $0.6832 \mathrm{E}-09$ \\
\hline 1.1 & $0.7275 E-08$ & $0.3619 \mathrm{E}-08$ & $0.2171 \mathrm{E}-08$ & $0.1314 \mathrm{E}-08$ & $0.7369 \mathrm{E}-09$ \\
\hline 1.2 & $0.7617 \mathrm{E}-08$ & 0.3777 E-08 & $0.2265 E-08$ & $0.1376 \mathrm{E}-08$ & $0.7765 \mathrm{E}-09$ \\
\hline 1.3 & $0.7852 E-08$ & $0.3881 \mathrm{E}-08$ & $0.2327 \mathrm{E}-08$ & $0.1418 \mathrm{E}-08$ & $0.8041 \mathrm{E}-09$ \\
\hline 1.4 & $0.7998 \mathrm{E}-08$ & $0.3942 E-08$ & $0.2363 E-08$ & $0.1443 E-08$ & $0.8221 \mathrm{E}-09$ \\
\hline 1.5 & $0.8074 \mathrm{E}-08$ & $0.3969 \mathrm{E}-08$ & $0.2379 E-08$ & $0.1455 \mathrm{E}-08$ & $0.8321 \mathrm{E}-09$ \\
\hline 1.6 & $0.8094 E-08$ & $0.3970 \mathrm{E}-08$ & $0.2378 \varepsilon-08$ & $0.1457 \varepsilon-08$ & $0.8360 \mathrm{E}-09$ \\
\hline 1.7 & $0.8070 \mathrm{E}-08$ & $0.3949 E-08$ & $0.2365 \varepsilon-08$ & $0.1451 E-08$ & $0.8348 \mathrm{E}-09$ \\
\hline 1.8 & $0.8012 \varepsilon-08$ & $0.3913 E-08$ & $0.2342 E-08$ & $0.1439 \mathrm{E}-08$ & $0.8299 \mathrm{E}-09$ \\
\hline 1.9 & $0.7928 \mathrm{E}-08$ & $0.3864 E-08$ & $0.2312 \varepsilon-08$ & $0.1422 E-08$ & $0.8219 \mathrm{E}-09$ \\
\hline 2.0 & $0.7823 \mathrm{E}-08$ & $0.3806 \mathrm{E}-08$ & $0.2276 \mathrm{E}-08$ & $0.1401 E-08$ & $0.8116 \mathrm{E}-09$ \\
\hline 2.1 & $0.7704 E-08$ & $0.3742 E-08$ & $0.2237 \varepsilon-08$ & $0.1378 \mathrm{E}-08$ & $0.7997 \mathrm{E}-09$ \\
\hline 2.2 & $0.7573 E-08$ & $0.3673 \mathrm{E}-08$ & $0.2194 \mathrm{E}-08$ & $0.1353 \mathrm{E}-08$ & $0.7864 \mathrm{E}-09$ \\
\hline 2.3 & $0.7434 \mathrm{E}-08$ & $0.3600 \mathrm{E}-08$ & $0.2150 E-08$ & $0.1327 \mathrm{E}-08$ & $0.7722 \mathrm{E}-09$ \\
\hline 2.4 & $0.7290 \mathrm{E}-08$ & $0.3526 \mathrm{E}-08$ & $0.2105 E-08$ & $0.1300 \varepsilon-08$ & $0.7574 \mathrm{E}-09$ \\
\hline 2.5 & $0.7143 E-08$ & $0.3450 \mathrm{E}-08$ & $0.2059 E-08$ & $0.1272 E-08$ & $0.7422 \mathrm{E}-09$ \\
\hline 2.6 & $0.6994 \mathrm{E}-08$ & $0.3374 E-08$ & $0.2013 \mathrm{E}-08$ & $0.1244 E-08$ & $0.7268 \mathrm{E}-09$ \\
\hline 2.7 & $0.6845 E-08$ & $0.3298 \mathrm{E}-08$ & $0.1967 \mathrm{E}-08$ & $0.1216 \mathrm{E}=08$ & $0.7113 \mathrm{E}-09$ \\
\hline 2.8 & $0.6696 \mathrm{E}-08$ & $0.3223 \mathrm{E}-08$ & $0.1922 \varepsilon-08$ & $0.1189 \mathrm{E}-08$ & $0.6958 \mathrm{E}-09$ \\
\hline 2.9 & $0.6549 \mathrm{E}-08$ & 0.31 & $0.1877 \mathrm{E}-08$ & $0.1161 \mathrm{E}-08$ & $0.6805 \mathrm{E}-09$ \\
\hline 3.0 & $0.6404 E-08$ & $0.3076 \mathrm{E}-08$ & $0.1833 \mathrm{E}-08$ & $0.1135 \mathrm{E}-08$ & $0.6654 \mathrm{E}-09$ \\
\hline 3.1 & $0.6261 \mathrm{E}-08$ & $0.3005 \mathrm{E}-08$ & $0.1790 \mathrm{E}-08$ & $0.1108 E-08$ & $0.6504 \mathrm{E}-09$ \\
\hline 3.2 & $0.6121 \mathrm{E}-08$ & 0.2 & 0.174 & $E-08$ & $0.6358 \mathrm{E}-09$ \\
\hline 3.3 & $0.5984 E-08$ & $7 \mathrm{E}-08$ & E-08 & $0.1058 \mathrm{E}-08$ & $0.6215 \mathrm{E}-09$ \\
\hline 3.4 & $0.5850 \mathrm{E}-08$ & $0.2800 E-08$ & $0.1667 \mathrm{E}-08$ & 0.10 & $0.6075 \mathrm{E}-09$ \\
\hline 3.5 & $0.5719 E-08$ & 0.2 & 0.16 & $E-08$ & $9 \mathrm{E}-09$ \\
\hline 3.6 & $0.5592 \mathrm{E}-08$ & $0.2673 \mathrm{E}-08$ & $0.1591 \mathrm{E}-08$ & $0.9862 E-09$ & $0.5806 \mathrm{E}-09$ \\
\hline 3.7 & $0.5469 \mathrm{E}-08$ & & & 0.9637 E-09 & $0.5676 \mathrm{E}-09$ \\
\hline 3.8 & $8 E-08$ & 0.2 & $E-08$ & $0.9419 \mathrm{E}-09$ & $0.5550 E-09$ \\
\hline 3.9 & $0.5231 E-08$ & $0.2496 \mathrm{E}-08$ & $0.1484 \mathrm{E}-08$ & $0.9207 \mathrm{E}-09$ & $0.5428 \mathrm{E}-09$ \\
\hline 4.0 & $0.5118 \mathrm{E}-08$ & 0.24 & $0.1451 \mathrm{E}-08$ & $0.9001 E-09$ & $0.5310 \mathrm{E}-09$ \\
\hline 4.1 & $0.5008 \mathrm{E}-08$ & $0.2386 E-08$ & $0.1418 \mathrm{E}-08$ & $0.8802 E-09$ & $0.5194 \mathrm{E}-09$ \\
\hline 4.2 & $0.4901 E-08$ & $0.2334 E-08$ & $0.1387 E-08$ & $0.8609 E-09$ & $0.5082 E-09$ \\
\hline 4.3 & $0.4797 \mathrm{E}-08$ & $0.2283 E-08$ & $0.1357 E-08$ & $0.8422 E-09$ & $0.4974 E-09$ \\
\hline 4.4 & $0.4696 \mathrm{E}-08$ & $0.2234 \mathrm{E}-08$ & $0.1327 \mathrm{E}-08$ & $0.8241 \mathrm{E}-09$ & $0.4869 \mathrm{E}-09$ \\
\hline 4.5 & $0.4599 \mathrm{E}-08$ & $0.2186 \mathrm{E}-08$ & $0.1299 \mathrm{E}-08$ & $0.8065 \mathrm{E}-09$ & $0.4767 E-09$ \\
\hline 4.6 & $0.4504 \mathrm{E}-08$ & $0.2140 E-08$ & $0.1271 \varepsilon-08$ & $0.7895 \mathrm{E}-09$ & $0.4668 \mathrm{E}-09$ \\
\hline 4.7 & $0.4412 E-08$ & $0.2096 \mathrm{E}-08$ & $0.1245 \mathrm{E}-08$ & $0.7730 \mathrm{E}-09$ & $0.4571 \mathrm{E}-09$ \\
\hline 4.8 & $0.4323 E-08$ & $0.2052 \varepsilon-08$ & $0.1219 \mathrm{E}-08$ & $0.7570 \mathrm{E}-09$ & $0.4478 \mathrm{E}-09$ \\
\hline 4.9 & $0.4236 \mathrm{E}-08$ & $0.2010 \mathrm{E}-08$ & $0.1194 \mathrm{E}-08$ & $0.74 .15 \mathrm{E}-09$ & $0.4388 \mathrm{E}-09$ \\
\hline 5.0 & $0.4152 E-08$ & $0.1970 \mathrm{E}-08$ & $0.1169 \mathrm{E}-08$ & $0.7265 E-09$ & $0.4300 \mathrm{E}-09$ \\
\hline
\end{tabular}


Table 3 e- $\mathrm{N}_{2}$ vibrational excitation rate coefficients, $\mathrm{cm}^{3} / \mathrm{s}$ $\left(v_{i}=0, J=150\right)$

\begin{tabular}{|c|c|c|c|c|c|}
\hline $\mathrm{r}, \mathrm{eV}$ & $v_{f}=1$ & 2 & 3 & 4 & 5 \\
\hline 0.1 & $0.1579 \mathrm{E}-11$ & $0.6654 E-12$ & $0.2366 \mathrm{E}-12$ & $0.6152 E-13$ & $0.1061 E-13$ \\
\hline 0.2 & $0.2560 \mathrm{E}-09$ & $0.1292 E-09$ & $0.6248 \mathrm{E}-10$ & $0.2511 \mathrm{E}-10$ & $0.7703 E-11$ \\
\hline 0.3 & $0.1310 \varepsilon-08$ & $0.6977 \mathrm{E}-09$ & $0.3652 E-09$ & $0.1680 E-09$ & $0.6203 E-10$ \\
\hline 0.4 & $0.2802 \mathrm{E}-08$ & $0.1525 \mathrm{E}-08$ & $0.8251 \mathrm{E}-09$ & $0.4045 \mathrm{E}-09$ & $0.1634 E-09$ \\
\hline 0.5 & $0.4231 E-08$ & $0.2324 E-08$ & $0.1280 \mathrm{E}-08$ & $0.6505 \mathrm{E}-09$ & $0.2770 \mathrm{E}-09$ \\
\hline 0.6 & $0.5382 E-08$ & $0.2966 \mathrm{E}-08$ & $0.1651 \mathrm{E}-08$ & $0.8590 \mathrm{E}-09$ & $0.3787 \varepsilon-09$ \\
\hline 0.7 & $0.6223 \mathrm{E}-08$ & $0.34328-08$ & $0.1924 \mathrm{E}-08$ & $0.1017 \mathrm{E}-08$ & $0.4595 \mathrm{E}-09$ \\
\hline 0.8 & $0.6791 E-08$ & $.3744 E-08$ & $0.2110 \mathrm{E}-08$ & $0.1128 \varepsilon-08$ & $0.5191 \mathrm{E}-09$ \\
\hline 0.9 & $0.7144 \mathrm{E}-08$ & $0.3934 E-08$ & $0.2225 E-08$ & $0.1201 \mathrm{E}-08$ & $0.5602 E-09$ \\
\hline 1.0 & $0.7333 E-08$ & $0.4033 E-08$ & $0.2287 E-08$ & $0.1243 E-08$ & $0.5864 E-09$ \\
\hline 1.1 & $0.7402 E-08$ & $0.4065 E-08$ & $0.2310 E-08$ & $0.1263 E-08$ & $0.6011 \mathrm{E}-09$ \\
\hline 1.2 & $0.7384 E-08$ & $0.4050 E-08$ & $0.2306 \mathrm{E}-08$ & $0.1266 \mathrm{E}-08$ & $0.6071 \mathrm{E}-09$ \\
\hline 1.3 & $0.7305 \varepsilon-08$ & $0.4001 E-08$ & $0.2281 \varepsilon-08$ & $0.1257 \mathrm{E}-08$ & $0.6067 \mathrm{E}-09$ \\
\hline 1.4 & $0.7183 E-08$ & $0.3928 \varepsilon-08$ & $0.2242 E-08$ & $0.1240 E-08$ & $0.6016 \mathrm{E}-09$ \\
\hline 1.5 & $0.7033 E-08$ & $0.3841 \mathrm{E}-08$ & $0.2194 E-08$ & $0.1217 \mathrm{E}-08$ & $0.5931 \mathrm{E}-09$ \\
\hline 1.6 & $0.6863 E-08$ & $0.3744 \mathrm{E}-08$ & $0.2140 \mathrm{E}-08$ & $0.1189 \mathrm{E}-08$ & $0.5823 \mathrm{E}-09$ \\
\hline 1.7 & $0.6682 \varepsilon-08$ & $0.3640 E-08$ & $0.2083 E-08$ & $0.1160 \mathrm{E}-08$ & $0.5699 \mathrm{E}-09$ \\
\hline 1.8 & $0.6495 \mathrm{E}-08$ & $0.3534 E-08$ & $0.2023 E-08$ & $0.1129 E-08$ & $0.5564 \mathrm{E}-09$ \\
\hline 1.9 & $0.6305 E-08$ & 3428 & $0.1963 E-08$ & $0.1097 \mathrm{E}-08$ & $0.5422 E-09$ \\
\hline 2.0 & 0.6117 E-08 & $0.3322 E-08$ & $0.1903 \mathrm{E}-08$ & $0.1065 \varepsilon-08$ & $0.5278 \varepsilon-09$ \\
\hline 2.1 & $0.5930 \mathrm{E}-08$ & $0.3217 \mathrm{E}$ & $0.1844 \mathrm{E}-08$ & $0.1034 \varepsilon-08$ & $0.51338-09$ \\
\hline 2.2 & $0.5748 \mathrm{E}-08$ & 0.3116 & $0.1786 \mathrm{E}-08$ & $0.1002 E-08$ & $0.4988 \mathrm{E}-09$ \\
\hline 2.3 & 0.55 & .3 & 0.1730 & 0.97 & $0.4846 \mathrm{E}-09$ \\
\hline 2.4 & $0.5398 \mathrm{E}-08$ & 0.2921 & $0.1676 \mathrm{E}-08$ & 0.94 & $0.4706 \mathrm{E}-09$ \\
\hline 2.5 & 0.5231 & .2829 & $0.1623 E-08$ & $E-09$ & $0.4570 \mathrm{E}-09$ \\
\hline 2.6 & $0.5071 \mathrm{E}-08$ & .2740 & $0.1573 E-08$ & $0.8858 \varepsilon-09$ & $0.4437 \varepsilon-09$ \\
\hline 2.7 & $0.4917 \varepsilon-08$ & $0.2655 \mathrm{E}-08$ & 0.1524 & E-09 & 0.43 \\
\hline 2.8 & $0.4768 E-08$ & .2573 & 0. & -09 & $0.4185 E-09$ \\
\hline 2.9 & $0.4626 E-08$ & ) & 0.143 & 09 & $6 E-09$ \\
\hline 3.0 & $0.4489 \mathrm{E}-08$ & & & 0. & -09 \\
\hline 3.1 & $0.4358 \mathrm{E}-08$ & 2 & $0.1348 \mathrm{E}-08$ & $0.7619 \mathrm{E}-09$ & $E-09$ \\
\hline 3.2 & $0.4232 E-08$ & 0.22 & 0.1 & & \\
\hline 3.3 & 0.41 & & 0.1 & 0. & 0.3 \\
\hline 3.4 & $0.3996 \mathrm{E}-08$ & ? & $E-08$ & $3 E-09$ & $0.3531 \mathrm{E}-09$ \\
\hline 3.5 & 0.3 & & & & $E-09$ \\
\hline 3.6 & 0.377 & 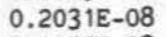 & $\varepsilon-08$ & 0.66 & $4 E-09$ \\
\hline 3.7 & $0.3677 \mathrm{E}-08$ & $0.1975 \mathrm{E}-08$ & 0.1 & & -09 \\
\hline 3.8 & & & 0.1 & 0. & 0.3 \\
\hline 3.9 & 0.3 & 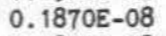 & 0.1 & $\varepsilon-09$ & $E-09$ \\
\hline 4.0 & $0.3395 E-08$ & & & & \\
\hline 4.1 & $0.3309 \varepsilon-08$ & & 0.1 & $E-09$ & $38 \mathrm{E}-09$ \\
\hline 4.2 & -08 & $0.1729 \mathrm{E}-08$ & $0.9938 \mathrm{E}-09$ & $0.56428-09$ & $0.2866 \mathrm{E}-09$ \\
\hline 4.3 & 0.31 & & $0.9689 \mathrm{E}-09$ & & $0.2796 \mathrm{E}-09$ \\
\hline 4.4 & $0.3069 \mathrm{E}-08$ & $0.1644 \mathrm{E}$ & $0.9450 E-$ & $8-09$ & E-09 \\
\hline 4.5 & $0.2995 E-08$ & $0.1604 \mathrm{E}-$ & $0.9220 \mathrm{E}-09$ & $3 E-09$ & $4 E-09$ \\
\hline 4.6 & $0.2924 E-08$ & $0.1565 \mathrm{E}-$ & $0.8998 \mathrm{E}-09$ & $0.5113 \mathrm{E}-09$ & $0.2602 E-09$ \\
\hline 4.7 & $0.2855 E-08$ & $0.1528 \mathrm{E}-$ & $0.8785 \mathrm{E}-09$ & $0.4994 E-09$ & $0.2542 E-09$ \\
\hline 4.8 & $0.2789 \mathrm{E}-08$ & $0.1493 E$ & $0.8580 \mathrm{E}-09$ & & $0.2485 E-09$ \\
\hline 4.9 & & & $0.8383 \mathrm{E}-$ & 0.47 & $0.2429 \mathrm{E}-09$ \\
\hline 5.0 & $0.2665 \mathrm{E}-08$ & $0.1425 E-08$ & $0.8192 \mathrm{E}-09$ & $0.4659 \mathrm{E}-09$ & $0.2375 E-09$ \\
\hline
\end{tabular}


Table 4 e-N 2 vibrational excitation rate coefficients, $\mathrm{cm}^{3} / \mathrm{s}$ $\left(v_{1}=1, J=50\right)$

\begin{tabular}{|c|c|c|c|c|c|}
\hline $\mathrm{T}, \mathrm{eV}$ & $v_{f}=2$ & 3 & 4 & 5 & 6 \\
\hline 0.1 & $0.8950 \mathrm{E}-13$ & $0.1546 \mathrm{E}-13$ & $0.2833 E-14$ & $0.4185 E-15$ & $0.5356 E-16$ \\
\hline 0.2 & $0.5077 \mathrm{E}-10$ & $0.1802 E-10$ & $0.5597 \mathrm{E}-11$ & $0.1601 \mathrm{E}-11$ & $0.5328 E-12$ \\
\hline 0.3 & $0.4252 E-09$ & $0.1763 \mathrm{E}-09$ & $0.6763 \mathrm{E}-10$ & $0.2574 E-10$ & $0.1156 \mathrm{E}-10$ \\
\hline 0.4 & $0.1788 \mathrm{E}-08$ & $0.5304 \mathrm{E}-09$ & $0.2288 \varepsilon-09$ & $0.1010 E-09$ & $0.5154 E-10$ \\
\hline 0.5 & $0.2153 \varepsilon-08$ & $0.1000 \mathrm{E}-08$ & $0.4631 \mathrm{E}-09$ & $0.2224 E-09$ & $0.1213 E-09$ \\
\hline 0.6 & $0.3146 \mathrm{E}-08$ & $0.1493 E-08$ & $0.7229 \mathrm{E}-09$ & $0.3660 \mathrm{E}-09$ & $0.2074 \mathrm{E}-09$ \\
\hline 0.7 & $0.4058 \mathrm{E}-08$ & $0.1950 \mathrm{E}-08$ & $0.9726 \mathrm{E}-09$ & $0.5099 \mathrm{E}-09$ & $0.2963 \mathrm{E}-09$ \\
\hline 0.8 & $0.4844 \mathrm{E}-08$ & $0.2344 E-08$ & $0.1193 E-08$ & $0.6409 \mathrm{E}-09$ & $0.3789 \mathrm{E}-09$ \\
\hline 0.9 & $0.5489 \mathrm{E}-08$ & $0.2666 \mathrm{E}-08$ & $0.1377 \mathrm{E}-08$ & $0.7530 \mathrm{E}-09$ & $0.4508 \mathrm{E}-09$ \\
\hline 1.0 & $0.6000 \mathrm{E}-08$ & $0.2919 \mathrm{E}-08$ & $0.1524 E-08$ & $0.8447 E-09$ & $0.5105 \mathrm{E}-09$ \\
\hline 1.1 & $0.6391 \mathrm{E}-08$ & $0.3111 \mathrm{E}-08$ & $0.1638 E-08$ & $0.9172 E-09$ & $0.5583 \mathrm{E}-09$ \\
\hline 1.2 & $0.6679 \mathrm{E}-08$ & $0.3251 \mathrm{E}-08$ & $0.1722 E-08$ & $0.9725 \mathrm{E}-09$ & $0.5954 E-09$ \\
\hline 1.3 & $0.6881 E-08$ & $0.3347 \mathrm{E}-08$ & $0.1782 E-08$ & $0.1013 \mathrm{E}-08$ & $0.6232 E-09$ \\
\hline 1.4 & $0.7012 \varepsilon-08$ & $0.3408 E-08$ & $0.1822 E-08$ & $0.1041 \mathrm{E}-08$ & $0.6431 \mathrm{E}-09$ \\
\hline 1.5 & $0.7086 E-08$ & $0.3441 E-08$ & $0.1845 \mathrm{E}-08$ & $0.1059 \mathrm{E}-08$ & $0.6564 \mathrm{E}-09$ \\
\hline 1.6 & $0.7113 E-08$ & $0.3450 \varepsilon-08$ & $0.1854 \mathrm{E}-08$ & $0.1069 \mathrm{E}-08$ & $0.6643 \mathrm{E}-09$ \\
\hline 1.7 & $0.7104 \mathrm{E}-08$ & $0.3441 \mathrm{E}-08$ & $0.1854 \mathrm{E}-08$ & $0.1072 E-08$ & $0.6678 \mathrm{E}-09$ \\
\hline 1.8 & $0.7065 \mathrm{E}-08$ & $0.3418 E-08$ & $0.1844 \mathrm{E}-08$ & $0.1070 E-08$ & $0.6678 \mathrm{E}-09$ \\
\hline 1.9 & $0.7003 E-08$ & $0.3384 E-08$ & $0.1829 \mathrm{E}-08$ & $0.1063 \varepsilon-08$ & $0.6650 \mathrm{E}-09$ \\
\hline 2.0 & $0.6924 E-08$ & $0.3342 E-08$ & $0.1808 \mathrm{E}-08$ & $0.1054 E-08$ & $0.6600 \mathrm{E}-09$ \\
\hline 2.1 & $0.6830 \mathrm{E}-08$ & $0.3293 E-08$ & $0.1783 \mathrm{E}-08$ & $0.1041 E-08$ & $0.6532 \varepsilon-09$ \\
\hline 2.2 & $0.6726 \mathrm{E}-08$ & $0.3239 \varepsilon-08$ & $0.1756 \mathrm{E}-08$ & $0.1027 \mathrm{E}-08$ & $0.6451 E-09$ \\
\hline 2.3 & $0.6615 \mathrm{E}-08$ & $0.3182 E-08$ & $0.1726 \mathrm{E}-08$ & $0.1011 \mathrm{E}-08$ & $0.6359 \mathrm{E}-09$ \\
\hline 2.4 & $0.6498 \varepsilon-08$ & $0.3122 E-08$ & $0.1695 E-08$ & $0.9943 \mathrm{E}-09$ & $0.6259 \varepsilon-09$ \\
\hline 2.5 & $0.6377 \varepsilon-08$ & $0.3061 E-08$ & $0.1663 E-08$ & $0.9766 \mathrm{E}-09$ & $0.6153 \mathrm{E}-09$ \\
\hline 2.6 & $0.6254 E-08$ & $0.2999 \mathrm{E}-08$ & $0.1630 \mathrm{E}-08$ & $0.9584 \mathrm{E}-09$ & $0.6044 E-09$ \\
\hline 2.7 & $0.6131 \mathrm{E}-08$ & $0.2937 \mathrm{E}-08$ & $0.1597 \mathrm{E}-08$ & $0.93998-09$ & $0.5932 E-09$ \\
\hline 2.8 & $0.6006 \mathrm{E}-08$ & 0.2875 & 0.156 & $0.9213 E-09$ & $0.5818 \mathrm{E}-09$ \\
\hline 2.9 & $0.5883 E-08$ & $0.2813 E-08$ & $0.1531 \mathrm{E}-08$ & $0.9026 \mathrm{E}-09$ & $0.5704 E-09$ \\
\hline 3.0 & $0.5760 E-08$ & $0.2752 E-08$ & 0.1 & $0.8840 \mathrm{E}-09$ & $0.5590 \mathrm{E}-09$ \\
\hline 3.1 & $0.5639 \mathrm{E}-08$ & 0.2 & 0.1 & $0.8656 E-09$ & $0.5477 \varepsilon-09$ \\
\hline 3.2 & $0.5520 \varepsilon-08$ & $0.2633 \mathrm{E}-08$ & $0.1434 E-08$ & $0.8474 E-09$ & $0.5365 E-09$ \\
\hline 3.3 & $0.5403 E-08$ & & 0.14 & 0.82 & $4 E-09$ \\
\hline 3.4 & $9 \mathrm{E}-08$ & -08 & 0.13 & $0.8120 \mathrm{E}-09$ & $0.5145 E-09$ \\
\hline 3.5 & $0.5177 \mathrm{E}-08$ & $0.2464 \mathrm{E}-08$ & $0.1343 E-08$ & $0.7948 \mathrm{E}-09$ & $0.5039 \mathrm{E}-09$ \\
\hline 3.6 & 0.5 & 0.24 & 0.131 & 0.7 & $E-09$ \\
\hline 3.7 & $0.4960 E-08$ & $0.2358 \varepsilon-08$ & $0.1285 \varepsilon-08$ & $0.7615 E-09$ & $0.4832 E-09$ \\
\hline 3.8 & $0.4856 E-08$ & 0.230 & & $E-09$ & \\
\hline 3.9 & 0.47 & 0.225 & 0.12 & $E-09$ & $0.4634 E-09$ \\
\hline 4.0 & $0.4656 \mathrm{E}-08$ & $0.2210 \mathrm{E}-08$ & $0.1205 E-08$ & $0.7146 \mathrm{E}-09$ & $0.4539 \varepsilon-09$ \\
\hline 4.1 & $0.4560 \mathrm{E}-08$ & $0.2163 E-08$ & $0.1179 \mathrm{E}-08$ & $0.6998 \mathrm{E}-09$ & $0.4446 \mathrm{E}-09$ \\
\hline 4.2 & $0.4466 \mathrm{E}-08$ & $0.2117 E-08$ & 0.115 & $0.6854 E-09$ & $0.4355 E-09$ \\
\hline 4.3 & $0.4375 E-08$ & $0.2073 E-08$ & $0.1130 \mathrm{E}-08$ & $0.6714 \mathrm{E}-09$ & $0.4267 \mathrm{E}-09$ \\
\hline 4.4 & $0.4287 E-08$ & $0.2030 \mathrm{E}-08$ & $0.1107 E-08$ & 0.6577 E-09 & $0.4182 E-09$ \\
\hline 4.5 & $0.4201 \mathrm{E}-08$ & $0.1989 \mathrm{E}-08$ & $0.1084 \mathrm{E}-08$ & $0.6445 \mathrm{E}-09$ & $0.4099 \varepsilon-09$ \\
\hline 4.6 & $0.4118 E-08$ & $0.1948 E-08$ & $0.1062 E-08$ & $0.6316 \mathrm{E}-09$ & $0.4017 \mathrm{E}-09$ \\
\hline 4.7 & $0.4037 \mathrm{E}-08$ & $0.1909 \mathrm{E}-08$ & $0.1041 E-08$ & $0.6191 \mathrm{E}-09$ & $0.3939 \mathrm{E}-09$ \\
\hline 4.8 & $0.3958 \mathrm{E}-08$ & $0.1871 \mathrm{E}-08$ & $0.1020 \mathrm{E}-08$ & $0.6069 \mathrm{E}-09$ & $0.3862 E-09$ \\
\hline 4.8 & $0.3882 E-08$ & $0.1834 E-08$ & $0.1000 \mathrm{E}-08$ & $0.5951 E-09$ & $0.3788 \mathrm{E}-09$ \\
\hline 5.0 & 0.3807 E- 08 & $0.1798 \mathrm{E}-08$ & $0.9807 E-09$ & $0.5836 \mathrm{E}-09$ & $0.3715 E-09$ \\
\hline
\end{tabular}


Table $5 e-N_{2}$ vibrational excitation rate coefficients, $\mathrm{cm}^{3} / \mathrm{s}$ $\left(v_{1}=2, J=50\right)$

\begin{tabular}{|c|c|c|c|c|c|}
\hline $\mathrm{T}, \mathrm{eV}$ & $v_{f}=3$ & 4 & 5 & 6 & 7 \\
\hline 0.1 & $0.2650 \mathrm{E}-12$ & $0.3850 E-13$ & $0.62458-14$ & $0.1059 \mathrm{E}-14$ & $0.1450 E-15$ \\
\hline 0.2 & $0.5822 \mathrm{E}-10$ & $1871 \mathrm{E}-10$ & $0.7244 \mathrm{E}-11$ & $0.2574 \mathrm{E}-11$ & $0.7299 \mathrm{E}-12$ \\
\hline 0.3 & $0.3624 \mathrm{E}-09$ & $0.1483 \mathrm{E}-09$ & $0.7358 \mathrm{E}-10$ & $0.3312 E-10$ & $0.1255 \mathrm{E}-10$ \\
\hline 0.4 & $0.9098 \mathrm{E}-09$ & $0.4151 \mathrm{E}-09$ & $0.2286 \mathrm{E}-09$ & $0.1159 \mathrm{E}-09$ & $0.5134 E-10$ \\
\hline 0.5 & $0.1590 \mathrm{E}-08$ & $0.7655 \mathrm{E}-09$ & $0.4429 \mathrm{E}-09$ & $0.2404 \mathrm{E}-09$ & $0.1169 \mathrm{E}-09$ \\
\hline 0.6 & $0.2305 E-08$ & $0.1140 \mathrm{E}-08$ & $0.6765 \mathrm{E}-09$ & $0.3829 \mathrm{E}-09$ & $0.1975 E-09$ \\
\hline 0.7 & $0.2987 \mathrm{E}-08$ & $0.1498 E-08$ & $0.9005 \mathrm{E}-09$ & $0.5238 \mathrm{E}-09$ & $0.2811 E-09$ \\
\hline 0.8 & $0.3598 \mathrm{E}-08$ & $0.1817 \mathrm{E}-08$ & $0.1099 \mathrm{E}-08$ & $0.6514 E-09$ & $0.3596 \mathrm{E}-09$ \\
\hline 0.9 & $0.4122 E-08$ & $0.2087 E-08$ & $0.1267 \mathrm{E}-08$ & $0.7606 \mathrm{E}-09$ & $0.4287 \mathrm{E}-09$ \\
\hline 1.0 & $0.4556 \mathrm{E}-08$ & $0.2309 \mathrm{E}-08$ & $0.1403 E-08$ & $0.8503 \mathrm{E}-09$ & $0.4869 \mathrm{E}-09$ \\
\hline 1.1 & $0.4905 \mathrm{E}-08$ & $0.2484 \mathrm{E}-08$ & $0.1509 \mathrm{E}-08$ & $0.9214 \mathrm{E}-09$ & $0.5342 E-09$ \\
\hline 1.2 & $0.5178 \mathrm{E}-08$ & $0.2619 E-08$ & $0.1590 \mathrm{E}-08$ & $0.9761 \mathrm{E}$ & $0.5715 \mathrm{E}-09$ \\
\hline 1.3 & $0.5384 E-08$ & $0.2718 E-08$ & $0.1649 \mathrm{E}-08$ & $0.1016 \mathrm{E}-08$ & $0.6000 \mathrm{E}-09$ \\
\hline 1.4 & $0.5533 \mathrm{E}-08$ & $0.2788 \mathrm{E}-08$ & $0.1689 \mathrm{E}-08$ & $0.1045 E-08$ & $0.6210 E-09$ \\
\hline 1.5 & $0.5634 \mathrm{E}-08$ & $0.2834 \mathrm{E}-08$ & $0.1714 E-08$ & $0.1063 \mathrm{E}-08$ & $0.6356 \mathrm{E}-09$ \\
\hline 1.6 & 0.5696 & 0.2859 & $0.1727 \mathrm{E}-08$ & $0.1074 E-08$ & $0.6449 \mathrm{E}-09$ \\
\hline 1.7 & $0.5726 \mathrm{E}-08$ & $0.2868 \mathrm{E}-08$ & $0.1730 \mathrm{E}-08$ & $0.1078 \mathrm{E}-08$ & $0.6499 \mathrm{E}-09$ \\
\hline 1.8 & $0.5728 E-08$ & $0.2864 E-08$ & $0.1725 E-08$ & $0.1076 \mathrm{E}-08$ & $0.6513 \mathrm{E}-09$ \\
\hline 1.9 & $0.5709 \mathrm{E}-08$ & $0.2849 \mathrm{E}-08$ & 0.1714 & $0.1071 E-08$ & 0.65 \\
\hline 2.0 & $0.5673 E-08$ & $0.2826 \mathrm{E}$ & 0.169 & 0.10 & 0.64 \\
\hline 2.1 & 0.5622 & 0.2 & 0.1677 & $0.10^{\circ}$ & 0.640 \\
\hline 2.2 & $0.5560 \varepsilon-08$ & $0.2761 \mathrm{E}-08$ & $0.1654 \mathrm{E}-08$ & $0.1036 \mathrm{E}-08$ & $0.6338 \mathrm{E}-09$ \\
\hline 2.3 & 0.5490 & $0.2721 \mathrm{E}-08$ & $0.1629 \mathrm{E}-08$ & $0.1021 E-08$ & $0.6258 \mathrm{E}-09$ \\
\hline 2.1 & 0.5413 & $0.2679 \mathrm{E}-08$ & 0.1602 & 0.1005 & $9 E-09$ \\
\hline 2.5 & $0.5331 \mathrm{E}-08$ & $0.2635 E-08$ & $0.1573 E-08$ & 00 & -09 \\
\hline 2.6 & 0.5245 & $0.2589 \mathrm{E}-08$ & $0.1544 \mathrm{E}-08$ & $0.9698 E-09$ & 0.59 \\
\hline 2.7 & $0.5157 \mathrm{E}-08$ & $0.2542 E-08$ & $0.1515 E-08$ & 0.9517 E-09 & $0.5869 \mathrm{E}-09$ \\
\hline 2.8 & $0.5067 \mathrm{E}-08$ & & 0.14 & & -09 \\
\hline 2.9 & 0.4976 & $0.2447 \mathrm{E}-08$ & 0.12 & 0.91 & 0.56 \\
\hline 3.0 & $0.4884 E-08$ & $0.2399 \mathrm{E}-08$ & 0.1426 & & $E-09$ \\
\hline 3.1 & 0.47 & 0.2 & 0.13 & 0.8 & 0.5 \\
\hline 3.2 & 0.47 & $0.2305 E-08$ & $0.1368 \mathrm{E}-08$ & $0.8605 E-09$ & -09 \\
\hline 3. & 0.46 & $0.2259 \mathrm{E}-08$ & 0.13 & & \\
\hline 3.4 & 0.45 & 0.2 & 0.13 & 0.8 & $E-09$ \\
\hline 3. & $0.4436 E-08$ & $0.2168 \mathrm{E}-08$ & $0.1284 E-08$ & $2 E-09$ & 0.502 \\
\hline & & & 0.12 & & 0. \\
\hline 3. & 0.42 & $0.2082 \mathrm{E}-08$ & 0.12 & $0.7751 E-09$ & 0.48 \\
\hline 3.8 & $0.4183 E-08$ & $0.2040 \mathrm{E}-08$ & 0.120 & & 0.472 \\
\hline & 0.41 & & 0.1 & & 0.1 \\
\hline $4 .(120$ & 0.40 & 0.195 & $0.1156 \mathrm{E}-08$ & $0.7283 \mathrm{E}-09$ & $0.4540 \mathrm{E}-09$ \\
\hline 4.1 & $0.3945 E-c$ & & & & \\
\hline 4.2 & 0.3870 & $0.1881 \mathrm{E}-0$ & 0.11 & $0.6990 E-09$ & $0.4362 \mathrm{E}-09$ \\
\hline 4.3 & $0.3796 \mathrm{E}-08$ & $0.1844 \mathrm{E}-08$ & $0.1087 \mathrm{E}-08$ & $0.6849 \mathrm{E}-09$ & $0.4276 \mathrm{E}-09$ \\
\hline 4.4 & $0.3724 \mathrm{E}-0$ & $0.1808 E-08$ & $0.1065 \varepsilon-08$ & $0.6712 E-09$ & $0.4192 E-09$ \\
\hline 4.5 & $0.3654 \mathrm{E}-\mathrm{C}$ & $0.1773 E-08$ & $0.1044 E-08$ & $0.6579 \mathrm{E}-09$ & $0.4111 \mathrm{E}-09$ \\
\hline 4.6 & $0.3585 E-08$ & $0.1739 \mathrm{E}-08$ & $E-08$ & $0.6449 \mathrm{E}-09$ & $0.4031 \mathrm{E}-09$ \\
\hline 4.7 & $0.3519 \mathrm{E}-\mathrm{C}$ & $0.1705 E-08$ & $0.1003 E-08$ & $0.6323 E-09$ & $0.3954 E-09$ \\
\hline 4.8 & $0.3454 \mathrm{E}-0$ & $0.1673 E-08$ & $0.9840 E-09$ & $0.6201 \mathrm{E}-09$ & $0.3879 \varepsilon-09$ \\
\hline 4.9 & 0.339 & 0. & & & \\
\hline$=$ & $0.3329 \mathrm{E}-$ & $0.1611 \mathrm{E}-\mathrm{C}$ & $0.9466 \mathrm{E}$ & $0.5966 \mathrm{E}-09$ & $0.3735 E-0$ \\
\hline
\end{tabular}


Table 6 e- $\mathrm{N}_{2}$ vibrational excitation rate coefficients, $\mathrm{cm}^{3} / \mathrm{s}$ $\left(v_{i}=3, J=50\right)$

\begin{tabular}{|c|c|c|c|c|c|}
\hline $\mathrm{T}, \mathrm{eV}$ & $v_{f}=4$ & 5 & 6 & 7 & 8 \\
\hline 0.1 & $0.6128 \mathrm{E}-12$ & $0.9426 \mathrm{E}-13$ & $0.1333 E-13$ & $0.1749 \mathrm{E}-14$ & $0.2419 E-15$ \\
\hline 0.2 & $0.7575 E-10$ & $0.2444 \mathrm{E}-10$ & $0.7585 E-11$ & $0.2578 \mathrm{E}-11$ & $0.8714 \mathrm{E}-12$ \\
\hline 0.3 & $0.3863 E-09$ & $0.1559 \mathrm{E}-09$ & $0.6416 \mathrm{E}-10$ & $0.2939 \mathrm{E}-10$ & $0.1312 E-10$ \\
\hline 0.4 & $0.8820 E-09$ & $0.3974 E-09$ & $0.1885 E-09$ & $0.9899 \mathrm{E}-10$ & $0.5059 \mathrm{E}-10$ \\
\hline 0.5 & $0.1469 E-08$ & $0.7021 \mathrm{E}-09$ & $0.3602 E-09$ & $0.2035 \mathrm{E}-09$ & $0.1122 E-09$ \\
\hline 0.6 & $0.2079 \mathrm{E}-08$ & $0.1026 \mathrm{E}-08$ & $0.5510 \mathrm{E}-09$ & $0.3247 \mathrm{E}-09$ & $0.1876 \mathrm{E}-09$ \\
\hline 0.7 & $0.2666 \mathrm{E}-08$ & $0.1339 \mathrm{E}-08$ & $0.7386 \mathrm{E}-09$ & $0.4467 \varepsilon-09$ & $0.2661 \mathrm{E}-09$ \\
\hline 0.8 & $0.3199 \mathrm{E}-08$ & $0.1622 E-08$ & $0.9096 \mathrm{E}-09$ & $0.5594 E-09$ & $0.3403 E-09$ \\
\hline 0.9 & $0.3664 \mathrm{E}-08$ & $0.1867 \mathrm{E}-08$ & $0.1058 \mathrm{E}-08$ & $0.6579 \mathrm{E}-09$ & $0.4063 E-09$ \\
\hline 1.0 & $0.4056 \mathrm{E}-08$ & $0.2071 \mathrm{E}-08$ & $0.1181 \mathrm{E}-08$ & $0.7404 E-09$ & $0.4624 E-09$ \\
\hline 1.1 & $0.4379 \mathrm{E}-08$ & $0.2236 \mathrm{E}-08$ & $0.1281 \mathrm{E}-08$ & $0.8074 \mathrm{E}-09$ & $0.5085 E-09$ \\
\hline 1.2 & $0.4636 E-08$ & $0.2367 \varepsilon-08$ & $0.1359 \mathrm{E}-08$ & $0.8601 \mathrm{E}-09$ & $0.5454 E-09$ \\
\hline 1.3 & $0.4836 \mathrm{E}-08$ & $0.2466 \mathrm{E}-08$ & $0.1419 \mathrm{E}-08$ & $0.9004 E-09$ & $0.5740 E-09$ \\
\hline 1.4 & $0.4987 E-08$ & $0.2540 \mathrm{E}-08$ & $0.1462 E-08$ & $0.9299 \varepsilon-09$ & $0.5954 E-09$ \\
\hline 1.5 & $0.5094 \mathrm{E}-08$ & $0.2590 \mathrm{E}-08$ & $0.1491 \mathrm{E}-08$ & $0.9504 \mathrm{E}-09$ & $0.6107 \mathrm{E}-09$ \\
\hline 1.6 & $0.5166 \mathrm{E}-08$ & $0.2622 E-08$ & $0.1510 \mathrm{E}-08$ & $0.9634 \mathrm{E}-09$ & $0.6209 \mathrm{E}-09$ \\
\hline 1.7 & $0.5208 \mathrm{E}-08$ & $0.2639 \mathrm{E}-08$ & $0.1519 \mathrm{E}-08$ & $0.9702 E-09$ & $0.6270 \mathrm{E}-09$ \\
\hline 1.8 & $0.5224 E-08$ & $0.2643 E-08$ & $0.1521 \mathrm{E}-08$ & $0.9720 \varepsilon-09$ & $0.6295 \mathrm{E}-09$ \\
\hline 1.9 & $0.5220 \mathrm{E}-08$ & $0.2637 \mathrm{E}$ & $0.1516 \mathrm{E}-08$ & $0.9697 \mathrm{E}-09$ & $0.6292 E-09$ \\
\hline 2.0 & $0.5199 \mathrm{E}-08$ & $0.2622 E-08$ & $0.1507 \mathrm{E}-08$ & $0.9642 E-09$ & $0.6266 \mathrm{E}-09$ \\
\hline 2.1 & $0.5165 \mathrm{E}-08$ & $0.2601 \mathrm{E}-08$ & $0.1494 \mathrm{E}-08$ & $0.9560 \mathrm{E}-09$ & $0.6222 E-09$ \\
\hline 2.2 & $0.5119 \mathrm{E}-08$ & $0.2574 E-08$ & $0.1477 \mathrm{E}-08$ & $0.9457 \mathrm{E}-09$ & $0.6163 E-09$ \\
\hline 2.3 & $0.5064 E-08$ & $0.2543 \mathrm{E}$ & 0.145 & $0.9339 \mathrm{E}-09$ & $0.6093 E-09$ \\
\hline 2.4 & $0.5003 E-08$ & $0.2508 \mathrm{E}-08$ & $0.1438 \mathrm{E}-08$ & $0.9207 \mathrm{E}-09$ & $0.6013 \mathrm{E}-09$ \\
\hline 2.5 & $0.4936 \mathrm{E}-08$ & $0.2471 \mathrm{E}-08$ & $0.1416 \mathrm{E}-08$ & $0.9067 \mathrm{E}-09$ & $0.5927 \mathrm{E}-09$ \\
\hline 2.6 & $0.4865 E-08$ & $0.2433 E-08$ & $0.1393 E-08$ & $0.8919 E-09$ & $0.5835 E-09$ \\
\hline 2.7 & $0.4790 \mathrm{E}-08$ & $0.2393 \mathrm{E}-08$ & $0.1369 \mathrm{E}-08$ & $0.8766 \mathrm{E}-09$ & $0.5739 \mathrm{E}-09$ \\
\hline 2.8 & $0.4714 \mathrm{E}-08$ & $0.2352 \mathrm{E}-08$ & $0.1345 \mathrm{E}-08$ & 0.8611 E-09 & $0.5641 \mathrm{E}-09$ \\
\hline 2.9 & $0.4635 \mathrm{E}-08$ & 0.23108 & $0.1320 \mathrm{E}-08$ & $0.8453 \mathrm{E}-09$ & $0.5541 E-09$ \\
\hline 3.0 & $0.4556 \mathrm{E}-08$ & $0.2268 \mathrm{E}-08$ & $0.1295 \mathrm{E}-08$ & $0.8295 E-09$ & $0.5441 \mathrm{E}-09$ \\
\hline 3.1 & $0.4477 \mathrm{E}-08$ & & $0.1271 \mathrm{E}-$ & $0.8137 E-09$ & $0.5340 \mathrm{E}-09$ \\
\hline 3.2 & 0.43 & $0.2185 \mathrm{E}-08$ & $0.1246 \mathrm{E}-08$ & $0.7980 \mathrm{E}-09$ & $0.5239 \mathrm{E}-09$ \\
\hline 3.3 & $0.4318 \mathrm{E}-08$ & $0.2144 E-08$ & $0.1222 E-08$ & $0.7824 E-09$ & $0.5139 \mathrm{E}-09$ \\
\hline 3.4 & 0.424 & 0.2 & 0.119 & E-09 & $0 E-09$ \\
\hline 3.5 & $0.4162 E-08$ & $2 E-08$ & $0.1175 \mathrm{E}-08$ & $0.7519 \mathrm{E}-09$ & $0.4943 \mathrm{E}-09$ \\
\hline 3.6 & $0.4086 E-08$ & & 0.11 & E-09 & $0.4846 \mathrm{E}-09$ \\
\hline 3.7 & $0.4010 \mathrm{E}-08$ & 0.19 & $0.1129 \mathrm{E}-08$ & $0.7224 \mathrm{E}-09$ & $0.4752 E-09$ \\
\hline 3.8 & $0.3936 \mathrm{E}-08$ & $0.1946 \mathrm{E}-08$ & $0.1107 \mathrm{E}-08$ & $\varepsilon-09$ & $E-09$ \\
\hline 3.9 & $0.3863 E-08$ & $0.1908 \mathrm{E}-08$ & $0.1085 E-08$ & 0.69 & $0.4568 \mathrm{E}-09$ \\
\hline 4.0 & 0.3 & 0.18 & $0.1063 \mathrm{E}-08$ & $0.6804 E-09$ & $0.4480 \varepsilon-09$ \\
\hline 4.1 & 0.37 & 0.18 & $0.1043 \mathrm{E}-08$ & $0.6670 \mathrm{E}-09$ & $0.4393 \mathrm{E}-09$ \\
\hline 4.2 & & $0.1801 \mathrm{E}-08$ & $0.1022 E-08$ & $0.6540 \mathrm{E}-09$ & $0.4308 \varepsilon-09$ \\
\hline 4.3 & $0.3586 \mathrm{E}-08$ & $0.1767 \varepsilon-08$ & $0.1003 E-08$ & $0.6413 \mathrm{E}-09$ & $0.4225 \mathrm{E}-09$ \\
\hline 4.4 & & $0.1733 E-08$ & $0.9833 \mathrm{E}-09$ & & $0.4144 E-09$ \\
\hline 4.5 & $0.3456 \mathrm{E}-08$ & $0.1701 \mathrm{E}-$ & $0.9644 \mathrm{E}-09$ & $0.6168 \mathrm{E}-09$ & $0.4065 \mathrm{E}-09$ \\
\hline 4.6 & $0.3394 \mathrm{E}-08$ & $0.1669 \mathrm{E}-08$ & $0.9461 \mathrm{E}-09$ & 0.6050 E-09 & $0.3988 \mathrm{E}-09$ \\
\hline 4.7 & $0.3332 E-08$ & $0.1638 \mathrm{E}-08$ & $0.9282 E-09$ & $0.5935 \mathrm{E}-09$ & $0.3913 \mathrm{E}-09$ \\
\hline 4.8 & $0.3273 E-08$ & $0.1608 \mathrm{E}-08$ & $0.9108 \mathrm{E}-09$ & $0.5823 \mathrm{E}-09$ & $0.3840 E-09$ \\
\hline 4.9 & $0.3215 \mathrm{E}-$ & $0.1579 \mathrm{E}-$ & $0.8939 \mathrm{E}-09$ & & $0.3769 \mathrm{E}-09$ \\
\hline 5 & $0.3158 \mathrm{E}-0$ & $0.1550 \mathrm{E}-$ & $0.8774 \mathrm{E}-$ & $0.5609 \mathrm{E}-09$ & $0.3700 E-0$ \\
\hline
\end{tabular}


Table 7 e- $\mathrm{N}_{2}$ vibrational excitation rate coefficients, $\mathrm{cm}^{3} / \mathrm{s}$ $\left(v_{1}=4, J=50\right)$

\begin{tabular}{|c|c|c|c|c|c|}
\hline $\mathrm{T}, \mathrm{eV}$ & $v_{f}=5$ & 6 & 7 & 8 & 9 \\
\hline 0.1 & $0.1168 \mathrm{E}-11$ & $0.1691 \mathrm{E}-12$ & $0.2417 \mathrm{E}-13$ & $0.2935 E-14$ & $0.3199 E-15$ \\
\hline 0.2 & $0.7765 \mathrm{E}-10$ & $0.2670 \mathrm{E}-10$ & $0.8934 E-11$ & $0.2591 \mathrm{E}-11$ & $0.7997 \mathrm{E}-12$ \\
\hline 0.3 & $0.3305 \mathrm{E}-09$ & $0.1480 E-09$ & $0.6523 \mathrm{E}-10$ & $0.2617 \mathrm{E}-10$ & $0.1122 E-10$ \\
\hline 0.4 & $0.7138 \mathrm{E}-09$ & $0.3569 \mathrm{E}-09$ & $0.1802 E-09$ & $0.8558 \mathrm{E}-10$ & $0.4291 E-10$ \\
\hline 0.5 & $0.1176 \mathrm{E}-08$ & $0.6178 \mathrm{E}-09$ & $0.3351 \mathrm{E}-09$ & $0.1754 \mathrm{E}-09$ & $0.9591 \mathrm{E}-10$ \\
\hline 0.6 & $0.1675 E-08$ & $0.8982 E-09$ & $0.5070 \mathrm{E}-09$ & $0.2814 \mathrm{E}-09$ & $0.1621 \mathrm{E}-09$ \\
\hline 0.7 & $0.2172 E-08$ & $0.1174 \mathrm{E}-08$ & $0.6772 E-09$ & $0.3901 \mathrm{E}-09$ & $0.2325 E-09$ \\
\hline 0.8 & $0.2639 \mathrm{E}-08$ & $0.1428 \mathrm{E}-08$ & $0.8341 \mathrm{E}-09$ & $0.4925 \mathrm{E}-09$ & $0.3004 E-09$ \\
\hline 0.9 & $0.3059 \mathrm{E}-08$ & $0.1653 E-08$ & $0.9717 \mathrm{E}-09$ & $0.5835 E-09$ & $0.3617 \mathrm{E}-09$ \\
\hline 1.0 & $0.3424 E-08$ & $0.1845 E-08$ & $0.1088 \mathrm{E}-08$ & $0.6612 \mathrm{E}-09$ & $0.4149 \mathrm{E}-09$ \\
\hline 1.1 & $0.3731 E-08$ & $0.2004 E-08$ & $0.1184 E-08$ & $0.7254 \mathrm{E}-09$ & $0.4593 E-09$ \\
\hline 1.2 & $0.3985 \mathrm{E}-08$ & $0.2132 E-08$ & $0.1260 \mathrm{E}-08$ & $0.7770 \mathrm{E}-09$ & $0.4955 E-09$ \\
\hline 1.3 & $0.4188 \varepsilon-08$ & $0.2233 E-08$ & $0.1319 E-08$ & $0.8172 \mathrm{E}-09$ & $0.5241 \mathrm{E}-09$ \\
\hline 1.4 & $0.4347 \mathrm{E}-08$ & $0.2310 \mathrm{E}-08$ & $0.1363 E-08$ & $0.8477 \mathrm{E}-09$ & $0.5461 \mathrm{E}-09$ \\
\hline 1.5 & $0.4468 \mathrm{E}-08$ & $0.2366 E-08$ & $0.1394 \mathrm{E}-08$ & $0.8697 \mathrm{E}-09$ & $0.5624 E-09$ \\
\hline 1.6 & $0.4555 E-08$ & $0.2405 E-08$ & $0.1415 \mathrm{E}-08$ & $0.8847 \mathrm{E}-09$ & $0.5739 \mathrm{E}-09$ \\
\hline 1.7 & $0.4614 E-08$ & $0.2429 \varepsilon-08$ & $0.1427 \mathrm{E}-08$ & $0.8938 \mathrm{E}-09$ & $0.5813 \varepsilon-09$ \\
\hline 1.8 & $0.4648 E-08$ & $0.2441 E-08$ & $0.1432 E-08$ & $0.8980 \mathrm{E}-09$ & $0.5854 E-09$ \\
\hline 1.9 & $0.4663 E-08$ & $0.2442 E-08$ & $0.1431 \mathrm{E}-08$ & $0.8983 \mathrm{E}-09$ & $0.5867 \mathrm{E}-09$ \\
\hline 2.0 & $0.4661 \mathrm{E}-08$ & $0.2435 E-08$ & $0.1425 \mathrm{E}-08$ & $0.8953 \mathrm{E}-09$ & $0.5857 \mathrm{E}-09$ \\
\hline 2.1 & $0.4645 \mathrm{E}-08$ & $0.2422 E-08$ & $0.1415 \mathrm{E}-08$ & $0.8896 \mathrm{E}-09$ & $0.5828 \mathrm{E}-09$ \\
\hline 2.2 & $0.4618 E-08$ & $0.2403 E-08$ & $0.1402 E-08$ & $0.8819 E-09$ & $0.5785 E-09$ \\
\hline 2.3 & $0.4582 E-08$ & $0.2379 \mathrm{E}-08$ & $0.1386 \mathrm{E}-08$ & $0.8724 E-09$ & $9 E-09$ \\
\hline 2.4 & $0.4537 \mathrm{E}-08$ & $0.2352 E-08$ & $0.1368 \mathrm{E}-08$ & $0.8617 \mathrm{E}-09$ & $0.5664 E-09$ \\
\hline 2.5 & $0.4487 E-08$ & $0.2322 E-08$ & $0.1349 \mathrm{E}-08$ & $0.8499 E-09$ & $0.5592 E-09$ \\
\hline 2.6 & $0.4432 E-08$ & $0.2289 \mathrm{E}-08$ & $0.1329 \mathrm{E}-08$ & $0.8373 E-09$ & $0.5513 \mathrm{E}-09$ \\
\hline 2.7 & $0.4373 E-08$ & $0.2255 \mathrm{E}-08$ & $0.1308 \mathrm{E}-08$ & $0.8241 E-09$ & $0.5430 \mathrm{E}-09$ \\
\hline 2.8 & $0.4312 \mathrm{E}-08$ & $0.2220 \mathrm{E}-08$ & $0.1286 \mathrm{E}-08$ & $0.8106 \mathrm{E}-09$ & $0.5344 \mathrm{E}-09$ \\
\hline 2.9 & $0.4248 E-08$ & $0.2184 E-08$ & $0.1264 \mathrm{E}-08$ & $0.7967 \mathrm{E}-09$ & $0.5256 \mathrm{E}-09$ \\
\hline 3.0 & $0.4182 E-08$ & $0.2148 \mathrm{E}-08$ & $0.1242 E-08$ & 0. & -09 \\
\hline 3.1 & $0.4116 \varepsilon-08$ & $0.2111 \mathrm{E}-08$ & $0.1219 E-08$ & $0.7686 \mathrm{E}-09$ & $0.5076 \mathrm{E}-09$ \\
\hline 3.2 & $0.4049 E-08$ & $0.2074 \mathrm{E}-08$ & $0.1197 \mathrm{E}-08$ & $0.7545 \mathrm{E}-09$ & $0.4985 \mathrm{E}-09$ \\
\hline 3.3 & $0.3982 E-08$ & 0.2 & 0.1 & $\varepsilon-09$ & $0.4895 \mathrm{E}-09$ \\
\hline 3.4 & $0.3914 \mathrm{E}-08$ & $0.2001 \mathrm{E}-08$ & $0.1153 E-08$ & 0.7267 E-09 & $0.4805 \mathrm{E}-09$ \\
\hline 3.5 & 0.3847 E- 08 & $0.1965 E-08$ & 0.113 & & $0.4716 \mathrm{E}-09$ \\
\hline 3.6 & $0.3781 E-08$ & $0.1929 \mathrm{E}-08$ & 0.1 & 0. & $8 E-09$ \\
\hline 3.7 & $0.3715 E-08$ & $0.1894 \mathrm{E}-08$ & $0.1088 \mathrm{E}-08$ & $0.6861 \mathrm{E}-09$ & $0.4541 \mathrm{E}-09$ \\
\hline 3.8 & $0.3650 E-08$ & & & & $0.4455 E-09$ \\
\hline 3.9 & $0.3586 E-08$ & 0.18 & 0.1 & 0.66 & $0.4372 E-09$ \\
\hline 4.0 & $0.3523 E-08$ & $0.1791 E-08$ & $0.1028 E-08$ & $0.6476 E-09$ & $0.4289 \mathrm{E}-09$ \\
\hline 4.1 & $0.3462 E-08$ & $0.1759 E-08$ & $0.1008 E-08$ & $0.6353 \mathrm{E}-09$ & $0.4209 \mathrm{E}-09$ \\
\hline 4.2 & $0.3401 E-08$ & $0.1726 E$ & $0.9890 \mathrm{E}-09$ & $0.6232 \varepsilon-09$ & $0.4130 E-09$ \\
\hline 4.3 & $0.3341 E-08$ & $0.1695 \mathrm{E}-08$ & $0.9704 \mathrm{E}-09$ & & $0.4053 \mathrm{E}-09$ \\
\hline 4.4 & $0.3283 \mathrm{E}-08$ & $0.1664 \mathrm{E}-08$ & $0.9522 \mathrm{E}-09$ & $0.6000 \mathrm{E}-09$ & $0.3977 \mathrm{E}-09$ \\
\hline 4.5 & $0.3225 E-08$ & $0.1634 E-08$ & $0.9344 \mathrm{E}-09$ & $0.5887 E-09$ & $0.3904 E-09$ \\
\hline 4.6 & $0.3169 \mathrm{E}-08$ & 0.160 & $0.9171 \mathrm{E}-09$ & $0.5778 \mathrm{E}-09$ & $0.3832 E-09$ \\
\hline 4.7 & $0.3114 \mathrm{E}-08$ & $0.1576 \mathrm{E}-$ & $0.9003 E-09$ & $0.5671 \mathrm{E}-09$ & $0.3761 \varepsilon-09$ \\
\hline 4.8 & $0.3061 E-08$ & $0.1548 E-08$ & $0.8838 \mathrm{E}-09$ & $0.5567 \mathrm{E}-09$ & $0.3693 \mathrm{E}-09$ \\
\hline 4.9 & & 0. & $0.8677 \mathrm{E}$ & $6 \mathrm{E}-09$ & $0.3626 \mathrm{E}-09$ \\
\hline 50 & $0.2957 \mathrm{E}-0$ & $0.1494 \mathrm{E}-08$ & $0.8521 \mathrm{E}-09$ & $0.5367 \mathrm{E}-09$ & $0.3561 \mathrm{E}-09$ \\
\hline
\end{tabular}


Table 8 e- $\mathrm{N}_{2}$ vibrational excitation rate coefficients, $\mathrm{cm}^{3} / \mathrm{s}$ $\left(v_{i}=5, J=50\right)$

\begin{tabular}{|c|c|c|c|c|c|}
\hline $\mathrm{T}, \mathrm{eV}$ & $v_{f}=6$ & 7 & 8 & 9 & 10 \\
\hline 0.1 & $0.1928 E-11$ & $0.2638 E-12$ & $0.3466 \mathrm{E}-13$ & $0.4339 E-14$ & $0.4194 \mathrm{E}-15$ \\
\hline 0.2 & $0.8141 \mathrm{E}-10$ & $0.2528 \mathrm{E}-10$ & $0.8860 \mathrm{E}-11$ & $0.2733 E-11$ & $0.7377 \mathrm{E}-12$ \\
\hline 0.3 & $0.3028 \mathrm{E}-09$ & $0.1237 \mathrm{E}-09$ & $0.5874 E-10$ & $0.2505 E-10$ & $0.9848 E-11$ \\
\hline 0.4 & $0.6260 \mathrm{E}-09$ & $0.2910 \mathrm{E}-09$ & $0.1576 \mathrm{E}-09$ & $0.7931 \mathrm{E}-10$ & $0.3769 \mathrm{E}-10$ \\
\hline 0.5 & $0.1021 \mathrm{E}-08$ & $0.5062 E-09$ & $0.2917 \mathrm{E}-09$ & $0.1609 E-09$ & $0.8509 E-10$ \\
\hline 0.6 & $0.1456 E-08$ & $0.7462 E-09$ & $0.4428 \varepsilon-09$ & $0.2578 \mathrm{E}-09$ & $0.1454 E-09$ \\
\hline 0.7 & $0.1899 \mathrm{E}-08$ & $0.9899 \mathrm{E}-09$ & $0.5953 \mathrm{E}-09$ & $0.3582 E-09$ & $0.2105 E-09$ \\
\hline 0.8 & $0.2324 \mathrm{E}-08$ & $0.1221 \mathrm{E}-08$ & $0.7386 \mathrm{E}-09$ & $0.4538 \mathrm{E}-09$ & $0.2742 E-09$ \\
\hline 0.9 & $0.2713 E-08$ & $0.1431 \mathrm{E}-08$ & $0.8665 E-09$ & $0.5396 E-09$ & $0.3326 E-09$ \\
\hline 1.0 & $0.3056 \mathrm{E}-08$ & $0.1615 E-08$ & $0.9765 \mathrm{E}-09$ & $0.6137 \mathrm{E}-09$ & $0.3838 E-09$ \\
\hline 1.1 & $0.3351 \mathrm{E}-08$ & $0.1770 \mathrm{E}-08$ & $0.1068 \mathrm{E}-08$ & $0.6757 \varepsilon-09$ & $0.4272 E-09$ \\
\hline 1.2 & $0.3598 E-08$ & $0.1899 \varepsilon-08$ & $0.1143 E-08$ & $0.7262 E-09$ & $0.4629 E-09$ \\
\hline 1.3 & $0.3800 E-08$ & $0.2003 E-08$ & $0.1202 E-08$ & $0.7661 \mathrm{E}-09$ & $0.4916 \mathrm{E}-09$ \\
\hline 1.4 & $0.3962 E-08$ & $0.2085 \mathrm{E}-08$ & $0.1248 E-08$ & $0.7969 E-09$ & $0.5141 \mathrm{E}-09$ \\
\hline 1.5 & $0.4088 \mathrm{E}-08$ & $0.2147 \mathrm{E}-08$ & $0.1282 E-08$ & $0.8197 E-09$ & $0.5311 E-09$ \\
\hline 1.6 & $0.4182 E-08$ & $0.2193 E-08$ & $0.1306 \mathrm{E}-08$ & $0.8358 \mathrm{E}-09$ & $0.5434 \mathrm{E}-09$ \\
\hline 1.7 & $0.4250 \mathrm{E}-08$ & $0.2225 E-08$ & $0.1321 \mathrm{E}-08$ & $0.8462 E-09$ & $0.5518 \mathrm{E}-09$ \\
\hline 1.8 & $0.4294 \mathrm{E}-08$ & $0.2245 E-08$ & $0.1329 E-08$ & $0.8519 E-09$ & $0.5569 \mathrm{E}-09$ \\
\hline 1.9 & $0.4320 \mathrm{E}-08$ & $0.2254 \mathrm{E}-08$ & $0.1332 E-08$ & $0.8536 E-09$ & $0.5593 \mathrm{E}-09$ \\
\hline 2.0 & $0.4328 E-08$ & $0.2255 E-08$ & $0.1329 E-08$ & $0.8522 \varepsilon-09$ & $0.5594 \mathrm{E}-09$ \\
\hline 2.1 & $0.4323 E-08$ & $0.2249 E-08$ & $0.1323 E-08$ & $0.8481 E-09$ & $0.5576 E-09$ \\
\hline 2.2 & $0.4307 \mathrm{E}-08$ & $0.2237 E-08$ & $0.1313 E-08$ & $0.8420 E-09$ & $0.5543 E-09$ \\
\hline 2.3 & $0.4281 E-08$ & $0.2221 \mathrm{E}-08$ & $0.1301 E-08$ & $0.8341 \mathrm{E}-09$ & $0.5498 \mathrm{E}-09$ \\
\hline 2.4 & $0.4247 \mathrm{E}-08$ & $0.2201 \mathrm{E}-08$ & $0.1287 \mathrm{E}-08$ & $0.8248 E-09$ & $0.5443 E-09$ \\
\hline 2.5 & $0.4207 E-08$ & $0.2177 \mathrm{E}-08$ & $0.1271 \mathrm{E}-08$ & $0.8144 E-09$ & $0.538 C E-09$ \\
\hline 2.6 & $0.4161 \mathrm{E}-08$ & $0.2151 \mathrm{E}-08$ & $0.1254 \mathrm{E}-08$ & $0.8033 E-09$ & $0.5310 E-09$ \\
\hline 2.7 & $0.4112 E-08$ & $0.2123 E-08$ & $0.1236 E-08$ & $0.7914 E-09$ & $0.5236 \mathrm{E}-09$ \\
\hline 2.8 & $0.4059 \mathrm{E}-08$ & $0.2094 \mathrm{E}-08$ & $0.1217 \mathrm{E}-08$ & $0.7792 \mathrm{E}-09$ & $0.5159 E-09$ \\
\hline 2.9 & $0.4004 E-08$ & $0.2063 E-08$ & $0.1198 \mathrm{E}-08$ & $0.7665 E-09$ & $0.5078 \mathrm{E}-09$ \\
\hline 3.0 & $0.3947 \mathrm{E}-08$ & $0.2032 E-08$ & $0.1178 E-08$ & $0.7537 \mathrm{E}-09$ & $0.4996 \mathrm{E}-09$ \\
\hline 3.1 & $0.3889 \mathrm{E}-08$ & $0.2000 \mathrm{E}-08$ & $0.1158 \mathrm{E}-08$ & $0.7407 E-09$ & $0.4913 E-09$ \\
\hline 3.2 & $0.3829 \mathrm{E}-08$ & $0.1968 \mathrm{E}-08$ & $0.1138 \mathrm{E}-08$ & $0.7277 E-09$ & $0.4829 E-09$ \\
\hline 3.3 & $0.3769 E-08$ & $0.1935 E-08$ & $0.1118 \mathrm{E}-08$ & $0.7147 \mathrm{E}-09$ & $0.4744 \mathrm{E}-09$ \\
\hline 3.4 & $0.3709 \mathrm{E}-08$ & $0.1903 \mathrm{E}-08$ & $0.1098 \mathrm{E}-08$ & $0.7018 \mathrm{E}-09$ & $0.4660 \mathrm{E}-09$ \\
\hline 3.5 & $0.3649 \mathrm{E}-08$ & $0.1871 \mathrm{E}-08$ & $0.1078 E-08$ & $0.6890 E-09$ & $0.4577 \mathrm{E}-09$ \\
\hline 3.6 & $0.3589 \mathrm{E}-08$ & $0.1838 \mathrm{E}-08$ & $0.1059 \mathrm{E}-08$ & $0.6763 E-09$ & $0.4494 E-09$ \\
\hline 3.7 & $0.3529 E-08$ & $0.1807 E-08$ & $0.1039 \mathrm{E}-08$ & $0.6638 \mathrm{E}-09$ & $0.4413 \mathrm{E}-09$ \\
\hline 3.8 & $0.3470 E-08$ & $0.1775 \varepsilon-08$ & $0.1020 \mathrm{E}-08$ & $0.6515 \mathrm{E}-09$ & $0.4332 E-09$ \\
\hline 3.9 & $0.3412 E-08$ & $0.1744 \mathrm{E}-08$ & $0.1002 E-08$ & $0.6394 \mathrm{E}-09$ & $0.4253 E-09$ \\
\hline 4.0 & $0.3354 \mathrm{E}-08$ & $0.1714 E-08$ & $0.9835 \mathrm{E}-09$ & $0.6276 \mathrm{E}-09$ & $0.4175 \mathrm{E}-09$ \\
\hline 4.1 & $0.3297 \mathrm{E}-08$ & $0.1684 E-08$ & $0.9655 E-09$ & $0.6159 \mathrm{E}-09$ & $0.4098 \mathrm{E}-09$ \\
\hline 4.2 & $0.3242 E-08$ & $0.1654 E-08$ & $0.9478 \mathrm{E}-09$ & $0.6045 E-09$ & $0.4023 E-09$ \\
\hline 4.3 & $0.3187 \varepsilon-08$ & $0.1625 E-08$ & $0.9306 \mathrm{E}-09$ & $0.5934 E-09$ & $0.3950 E-09$ \\
\hline 4.4 & $0.3133 E-08$ & $0.1597 \mathrm{E}-08$ & $0.9137 \mathrm{E}-09$ & $0.5825 E-09$ & $0.3878 E-09$ \\
\hline 4.5 & $0.3080 \mathrm{E}-08$ & $0.1569 \varepsilon-08$ & $0.8972 E-09$ & $0.5718 \mathrm{E}-09$ & $0.3808 E-09$ \\
\hline 4.6 & $0.3028 \mathrm{E}-08$ & $0.1542 E-08$ & $0.8810 \mathrm{E}-09$ & $0.5614 \mathrm{E}-09$ & $0.3739 \mathrm{E}-09$ \\
\hline 4.7 & $0.2977 \varepsilon-08$ & $0.1515 \mathrm{E}-08$ & $0.8653 E-09$ & $0.5513 E-09$ & $0.3672 E-09$ \\
\hline 4.8 & $0.2927 \mathrm{E}-08$ & $0.1489 \mathrm{E}-08$ & $0.8499 E-09$ & $0.5413 E-09$ & $0.3606 \mathrm{E}-09$ \\
\hline 4.9 & $0.2878 E-08$ & $0.1463 E-08$ & $0.8348 E-09$ & $0.5316 \mathrm{E}-09$ & $0.3542 E-09$ \\
\hline 5.0 & $0.2830 \mathrm{E}-08$ & $0.1439 \mathrm{E}-08$ & $0.8201 E-09$ & $0.5222 \mathrm{E}-09$ & $0.3480 E-09$ \\
\hline
\end{tabular}


Table 9 e- $\mathrm{N}_{2}$ vibrational excitation rate coefficients, $\mathrm{cm}^{3} / \mathrm{s}$ $\left(v_{i}=6, J=50\right)$

$\mathrm{T}, \mathrm{eV} \quad \mathrm{v}_{\mathrm{f}}=7$ 8 9 10 11

\begin{tabular}{|c|c|c|c|c|c|}
\hline 0.1 & $0.2675 E-11$ & $0.3642 E-12$ & $0.4395 E-13$ & $0.5213 E-14$ & $0.5194 E-15$ \\
\hline 0.2 & $0.7817 \mathrm{E}-10$ & $0.2433 E-10$ & $0.7795 \mathrm{E}-11$ & $0.2547 \mathrm{E}-11$ & $0.7058 \mathrm{E}-12$ \\
\hline 0.3 & $0.2669 \mathrm{E}-09$ & $0.1092 \mathrm{E}-09$ & $0.4853 \mathrm{E}-10$ & $0.2218 \mathrm{E}-10$ & $0.9047 \mathrm{E}-11$ \\
\hline 0.4 & $0.54+8 E-09$ & $0.2526 \mathrm{E}-09$ & $0.1304 \mathrm{E}-09$ & $0.6983 E-10$ & $0.3449 E-10$ \\
\hline 0.5 & $0.8852 \mathrm{E}-09$ & $0.4412 E-09$ & $0.2451 \mathrm{E}-09$ & $0.1425 \mathrm{E}-09$ & $0.7819 E-10$ \\
\hline 0.6 & $0.1273 E-08$ & $0.6566 \mathrm{E}-09$ & $0.3782 E-09$ & $0.2302 \varepsilon-09$ & $0.1344 \mathrm{E}-09$ \\
\hline 0.7 & $0.1676 \mathrm{E}-08$ & $0.8799 \mathrm{E}-09$ & $0.5160 \mathrm{E}-09$ & $0.3225 \mathrm{E}-09$ & $0.1957 \mathrm{E}-09$ \\
\hline 0.8 & $0.2068 \mathrm{E}-08$ & $0.1096 \mathrm{E}-08$ & $0.6481 E-09$ & $0.4115 E-09$ & $0.2562 \mathrm{E}-09$ \\
\hline 0.9 & $0.2433 E-08$ & $0.1295 \mathrm{E}-08$ & $0.7684 \mathrm{E}-09$ & $0.4925 E-09$ & $0.3122 E-09$ \\
\hline 1.0 & $0.2760 \mathrm{E}-08$ & $0.1472 E-08$ & $0.8737 \mathrm{E}-09$ & $0.5633 \mathrm{E}-09$ & $0.3617 \mathrm{E}-09$ \\
\hline 1.1 & $0.3045 \mathrm{E}-08$ & $0.1624 E-08$ & $0.9632 E-09$ & $0.6233 \mathrm{E}-09$ & $0.4041 E-09$ \\
\hline 1.2 & $0.3286 \mathrm{E}-08$ & $0.1753 E-08$ & $0.1037 \mathrm{E}-08$ & $0.6727 \mathrm{E}-09$ & $0.4393 E-09$ \\
\hline 1.3 & $0.3487 \mathrm{E}-08$ & $0.1858 \mathrm{E}-08$ & $0.1097 \mathrm{E}-08$ & $0.7125 \mathrm{E}-09$ & $0.4679 \mathrm{E}-09$ \\
\hline 1.4 & $0.3650 \mathrm{E}-08$ & $0.1942 E-08$ & $0.1145 E-08$ & $0.7436 \mathrm{E}-09$ & $0.4905 E-09$ \\
\hline 1.5 & $0.3779 E-08$ & $0.2009 \mathrm{E}-08$ & $0.1181 \mathrm{E}-08$ & $0.7672 E-09$ & $0.5079 \mathrm{E}-09$ \\
\hline 1.6 & $0.3879 \mathrm{E}-08$ & $0.2059 \mathrm{E}-08$ & $0.1207 \mathrm{E}-08$ & $0.7844 \mathrm{E}-09$ & $0.5208 \mathrm{E}-09$ \\
\hline 1.7 & $0.3953 \mathrm{E}-08$ & $0.2095 E-08$ & $0.1226 \mathrm{E}-08$ & $0.7962 E-09$ & $0.5298 \mathrm{E}-09$ \\
\hline 1.8 & $0.4005 \mathrm{E}-08$ & $0.2120 E-08$ & $0.1237 \mathrm{E}-08$ & $0.8033 \mathrm{E}-09$ & $0.5357 \mathrm{E}-09$ \\
\hline 1.9 & $0.4038 \mathrm{E}-08$ & $0.2134 \mathrm{E}-08$ & $0.1243 E-08$ & $0.8066 \mathrm{E}-09$ & $0.5388 \mathrm{E}-09$ \\
\hline 2.0 & $0.4055 E-08$ & $0.2140 \mathrm{E}-08$ & $0.1244 E-08$ & $0.8068 \mathrm{E}-09$ & $0.5396 \mathrm{E}-09$ \\
\hline 2.1 & $0.4058 \mathrm{E}-08$ & $0.2140 \mathrm{E}-08$ & $0.1241 E-08$ & $0.8043 E-09$ & $0.5386 \mathrm{E}-09$ \\
\hline 2.2 & $0.4050 \mathrm{E}-08$ & $0.2133 \mathrm{E}-08$ & $0.1235 E-08$ & $0.7997 \mathrm{E}-09$ & $0.5361 \mathrm{E}-09$ \\
\hline 2.3 & $0.4032 E-08$ & $0.2121 \mathrm{E}-08$ & $0.1226 E-08$ & $0.7934 \mathrm{E}-09$ & $0.5323 E-09$ \\
\hline 2.4 & $0.4006 \mathrm{E}-08$ & $0.2105 E-08$ & $0.1215 E-08$ & $0.7856 \mathrm{E}-09$ & $0.5275 E-09$ \\
\hline 2.5 & $0.3974 \mathrm{E}-08$ & $0.2086 E-08$ & $0.1202 E-08$ & $0.7767 \mathrm{E}-09$ & $0.5219 E-09$ \\
\hline 2.6 & $0.3937 \mathrm{E}-08$ & $0.2064 E-08$ & $0.1187 \mathrm{E}-08$ & $0.7669 \mathrm{E}-09$ & $0.5156 \mathrm{E}-09$ \\
\hline 2.7 & $0.3895 \mathrm{E}-08$ & $0.2040 \mathrm{E}-08$ & $0.1172 E-08$ & $0.7565 E-09$ & $0.5088 \mathrm{E}-09$ \\
\hline 2.8 & $0.3849 \mathrm{E}-08$ & $0.2014 \mathrm{E}-08$ & $0.1156 \mathrm{E}-08$ & $0.7455 \mathrm{E}-09$ & $0.5017 \mathrm{E}-09$ \\
\hline 2.9 & $0.3801 E-08$ & $0.1987 E-08$ & $0.1139 E-08$ & $0.7341 \mathrm{E}-09$ & $0.4942 E-09$ \\
\hline 3.0 & $0.3751 E-08$ & $0.1959 \mathrm{E}-08$ & $0.1121 \mathrm{E}-08$ & $0.7225 \mathrm{E}-09$ & $0.4865 E-09$ \\
\hline 3.1 & $0.3699 \mathrm{E}-08$ & $0.1931 \mathrm{E}-08$ & $0.1104 E-08$ & $0.7106 \mathrm{E}-09$ & $0.4787 \mathrm{E}-09$ \\
\hline 3.2 & $0.3645 E-08$ & $0.1901 \mathrm{E}-08$ & $0.1086 E-08$ & $0.6987 \mathrm{E}-09$ & $0.4708 \mathrm{E}-09$ \\
\hline 3.3 & $0.3591 \mathrm{E}-08$ & $0.1872 E-08$ & $0.1068 \mathrm{E}-08$ & $0.6867 \mathrm{E}-09$ & $0.4629 \mathrm{E}-09$ \\
\hline 3.4 & $0.3537 \mathrm{E}-08$ & $0.1842 E-08$ & $0.1050 E-08$ & $0.6748 \mathrm{E}-09$ & $0.4549 \mathrm{E}-09$ \\
\hline 3.5 & $0.3482 E-08$ & $0.1812 E-08$ & $0.1032 E-08$ & $0.6629 \mathrm{E}-09$ & $0.4470 E-09$ \\
\hline 3 & $0.3427 \mathrm{E}-08$ & $0.1783 E-08$ & $0.1014 E-08$ & $0.6511 \mathrm{E}-09$ & $0.4391 \mathrm{E}-09$ \\
\hline 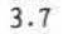 & $0.3373 E-08$ & $0.1753 E-08$ & $0.9961 \mathrm{E}-09$ & $5 E-09$ & $0.4314 E-09$ \\
\hline 3.8 & $0.3318 \mathrm{E}-08$ & $0.1724 \mathrm{E}-08$ & $0.9787 \mathrm{E}-09$ & $0.6280 \mathrm{E}-09$ & $7 E-09$ \\
\hline 3. & $0.3265 E-08$ & $0.1695 \mathrm{E}-08$ & $0.9614 \mathrm{E}-09$ & $0.6167 \mathrm{E}-09$ & $0.4161 \mathrm{E}-09$ \\
\hline & $0.3211 \mathrm{E}-08$ & $0.1666 \mathrm{E}-08$ & $0.9445 \mathrm{E}-09$ & $0.6056 \mathrm{E}-09$ & $0.4086 \mathrm{E}-09$ \\
\hline 4.1 & $0.3159 \mathrm{E}-08$ & $0.1638 \mathrm{E}-08$ & $0.9278 \mathrm{E}-09$ & $0.5946 \mathrm{E}-09$ & $0.4013 \mathrm{E}-09$ \\
\hline 4.2 & $0.3107 \mathrm{E}-08$ & $0.1611 \mathrm{E}-08$ & $0.9115 \mathrm{E}-09$ & $0.5839 \mathrm{E}-09$ & $0.3941 \mathrm{E}-09$ \\
\hline 4.3 & $0.3056 E-08$ & $0.1583 E-08$ & $0.8954 \mathrm{E}-09$ & $0.5734 \mathrm{E}-09$ & $0.3871 E-09$ \\
\hline 4.4 & $0.3005 E-08$ & $0.1556 \mathrm{E}-08$ & $0.8797 \mathrm{E}-09$ & $0.5631 \mathrm{E}-09$ & $0.3802 E-09$ \\
\hline 4.5 & $0.2956 \mathrm{E}-08$ & $0.1530 \mathrm{E}-08$ & $0.8642 \mathrm{E}-09$ & $0.5531 E-09$ & 0.3734 E-09 \\
\hline 4.6 & $0.2907 \mathrm{E}-08$ & $0.1504 E-08$ & $0.8491 \mathrm{E}-09$ & $0.5432 E-09$ & $0.3668 \mathrm{E}-09$ \\
\hline 4.7 & $0.2860 E-08$ & $0.1479 E-08$ & $0.8344 \mathrm{E}-09$ & $0.5336 \mathrm{E}-09$ & $0.3603 E-09$ \\
\hline 4.8 & $0.2813 E-08$ & $0.1454 \mathrm{E}-08$ & $0.8199 \mathrm{E}-09$ & $0.5242 \mathrm{E}-09$ & $0.3539 E-09$ \\
\hline 4.9 & $0.2767 \mathrm{E}-08$ & $0.1430 E-0$ & $0.8058 \mathrm{E}-09$ & $0.5150 E-09$ & $0.3478 \mathrm{E}-09$ \\
\hline 0.0 & $0.2722 E-08$ & $0.1406 \mathrm{E}-08$ & $0.7920 \mathrm{E}-09$ & $0.5060 \mathrm{E}-09$ & 0.3417 E-09 \\
\hline
\end{tabular}


Table 10 e-N 2 vibrational excitation rate coefficients, $\mathrm{cm}^{3} / \mathrm{s}$ $\left(v_{i}=7, J=50\right)$

\begin{tabular}{|c|c|c|c|c|c|}
\hline$r, \mathrm{eV}$ & $v_{f}=8$ & 9 & 10 & 11 & 12 \\
\hline 0.1 & $0.3242 E-11$ & $0.4331 E-12$ & $0.5151 \mathrm{E}-13$ & $0.5394 \mathrm{E}-14$ & $0.5564 E-15$ \\
\hline 0.2 & $0.6730 \mathrm{E}-10$ & $0.2234 E-10$ & $0.6888 \varepsilon-11$ & $0.2145 E-11$ & $0.6390 E-12$ \\
\hline 0.3 & $0.2178 \mathrm{E}-09$ & $0.9573 E-10$ & $0.4152 E-10$ & $0.1864 E-10$ & $0.8122 E-11$ \\
\hline 0.4 & $0.4467 \mathrm{E}-09$ & $0.2212 E-09$ & $0.1127 E-09$ & $0.5993 \mathrm{E}-10$ & $0.3125 E-10$ \\
\hline 0.5 & $0.7461 E-09$ & $0.3899 \mathrm{E}-09$ & $0.2148 \mathrm{E}-09$ & $0.1247 \mathrm{E}-09$ & $0.7152 E-10$ \\
\hline 0.6 & $0.1095 \mathrm{E}-08$ & $0.5867 \mathrm{E}-09$ & $0.3360 \mathrm{E}-09$ & $0.2045 E-09$ & $0.1239 \mathrm{E}-09$ \\
\hline 0.7 & $0.1466 \mathrm{E}-08$ & $0.7944 E-09$ & $0.4636 \mathrm{E}-09$ & $0.2898 \mathrm{E}-09$ & $0.1818 \mathrm{E}-09$ \\
\hline 0.8 & $0.1833 E-08$ & $0.9986 \mathrm{E}-09$ & $0.5878 \mathrm{E}-09$ & $0.3733 E-09$ & $0.2395 \varepsilon-09$ \\
\hline 0.9 & $0.2180 E-08$ & $0.1189 \mathrm{E}-08$ & $0.7024 \mathrm{E}-09$ & $0.4503 E-09$ & $0.2933 \varepsilon-09$ \\
\hline 1.0 & $0.2494 E-08$ & $0.1361 \mathrm{E}-08$ & $0.8041 E-09$ & $0.5184 E-09$ & $0.3413 E-09$ \\
\hline 1.1 & $0.2770 E-08$ & $0.1510 E-08$ & $0.8916 \mathrm{E}-09$ & 0.5767 E-09 & $0.3826 \mathrm{E}-09$ \\
\hline 1.2 & $0.3008 \mathrm{E}-08$ & $0.1638 E-08$ & $0.9650 \mathrm{E}-09$ & $0.6253 E-09$ & $0.4173 E-09$ \\
\hline 1.3 & $0.3207 E-08$ & $0.1744 E-08$ & $0.1025 E-08$ & $0.6649 \mathrm{E}-09$ & $0.4457 \mathrm{E}-09$ \\
\hline 1.4 & $0.3372 E-08$ & $0.1830 E-08$ & $0.1074 E-08$ & $0.6964 \varepsilon-09$ & $0.4685 \varepsilon-09$ \\
\hline 1.5 & $0.3505 E-08$ & $0.1899 \mathrm{E}-08$ & $0.1111 \mathrm{E}-08$ & $0.7207 E-09$ & $0.4861 \varepsilon-09$ \\
\hline 1.6 & $0.3609 E-08$ & $0.1953 E-08$ & $0.1140 E-08$ & $0.7389 \mathrm{E}-09$ & $0.4995 E-09$ \\
\hline 1.7 & $0.3689 E-08$ & $0.1993 E-08$ & $0.1160 E-08$ & $0.7517 \mathrm{E}-09$ & $0.5091 \mathrm{E}-09$ \\
\hline 1.8 & $0.3747 E-08$ & $0.2021 \varepsilon-08$ & $0.1174 \varepsilon-08$ & $0.7602 \varepsilon-09$ & $0.5155 \varepsilon-09$ \\
\hline 1.9 & $0.3787 \mathrm{E}-08$ & $0.2040 E-08$ & $0.1182 E-08$ & $0.7648 \mathrm{E}-09$ & $0.5192 E-09$ \\
\hline 2.0 & $0.3811 \mathrm{E}-08$ & $0.2050 E-08$ & $0.1186 E-08$ & $0.7663 \mathrm{E}-09$ & $0.5207 \mathrm{E}-09$ \\
\hline 2.1 & $0.3821 E-08$ & $0.2053 E-08$ & $0.1185 E-08$ & $0.7653 \varepsilon-09$ & $0.5204 E-09$ \\
\hline 2.2 & $0.3820 \mathrm{E}-08$ & $0.2050 \mathrm{E}-08$ & $0.1181 \mathrm{E}-08$ & $0.7620 \varepsilon-09$ & $0.5185 E-09$ \\
\hline 2.3 & $0.3810 E-08$ & $0.2042 E-08$ & $0.1174 \varepsilon-08$ & 0.7570 E-09 & $0.5154 E-09$ \\
\hline 2.4 & $0.3791 \mathrm{E}-08$ & $0.2030 E-08$ & $0.1165 \mathrm{E}-08$ & $0.7506 \varepsilon-09$ & $0.5113 E-09$ \\
\hline 2.5 & $0.3766 \varepsilon-08$ & $0.2014 E-08$ & $0.1155 E-08$ & $0.7430 \mathrm{E}-09$ & $0.5063 \mathrm{E}-09$ \\
\hline 2.6 & $0.3735 E-08$ & $0.1996 \mathrm{E}-08$ & $0.1142 E-08$ & $0.7345 E-09$ & $0.5006 \mathrm{E}-09$ \\
\hline 2.7 & $0.3700 \mathrm{E}-08$ & $0.1975 E-08$ & $0.1129 \mathrm{E}-08$ & $0.7252 E-09$ & $0.4944 \mathrm{E}-09$ \\
\hline 2.8 & $0.3661 \mathrm{E}-08$ & $0.1952 E-08$ & $0.1114 E-08$ & $0.7154 \mathrm{E}-09$ & $0.4878 \mathrm{E}-09$ \\
\hline 2.9 & $0.3618 \mathrm{E}-08$ & $0.1928 \mathrm{E}-08$ & $0.1099 \mathrm{E}-08$ & $0.7051 E-09$ & $0.4809 E-09$ \\
\hline 3.0 & $0.3574 E-08$ & $0.1903 E-08$ & $0.1083 \mathrm{E}-08$ & $0.6945 \mathrm{E}-09$ & $0.4737 \mathrm{E}-09$ \\
\hline 3.1 & $0.3528 E-08$ & $0.1877 \varepsilon-08$ & $0.1067 \mathrm{E}-08$ & $0.6836 \mathrm{E}-09$ & $0.4664 \mathrm{E}-09$ \\
\hline 3.2 & $0.3480 E-08$ & $0.1850 \mathrm{E}-08$ & $0.1051 E-08$ & $0.6727 E-09$ & $0.4590 \mathrm{E}-09$ \\
\hline 3.3 & $0.3431 E-08$ & $0.1823 E-08$ & $0.1034 \mathrm{E}-08$ & $0.6616 \mathrm{E}-09$ & $0.4515 E-09$ \\
\hline 3.4 & $0.3381 E-08$ & $0.1795 E-08$ & $0.1018 E-08$ & $0.6506 \mathrm{E}-09$ & $0.4439 \varepsilon-09$ \\
\hline 3.5 & $0.3331 E-08$ & $0.1767 E-08$ & $0.1001 E-08$ & $0.6395 E-09$ & $0.4364 E-09$ \\
\hline 3.6 & $0.3281 \mathrm{E}-08$ & $0.1740 E-08$ & $0.9842 E-09$ & $0.6285 E-09$ & $0.4289 E-09$ \\
\hline 3.7 & $0.3231 E-08$ & $0.1712 E-08$ & 0.9677 E-09 & $0.6177 \mathrm{E}-09$ & $0.4215 E-09$ \\
\hline 3.8 & $0.3181 \mathrm{E}-08$ & $0.1684 \mathrm{E}-08$ & $0.9514 E-09$ & $0.6069 \varepsilon-09$ & $0.4142 E-09$ \\
\hline 3.9 & $0.3131 E-08$ & $0.1657 E-08$ & $0.9352 E-09$ & $0.5963 \mathrm{E}-09$ & $0.4069 \mathrm{E}-09$ \\
\hline 4.0 & $0.3082 E-08$ & $0.1630 \mathrm{E}-08$ & $0.9192 E-09$ & $0.5858 \mathrm{E}-09$ & $0.3998 \mathrm{E}-09$ \\
\hline 4.1 & $0.3033 E-08$ & $0.1603 E-08$ & $0.9035 E-09$ & $0.5755 E-09$ & $0.3928 \mathrm{E}-09$ \\
\hline 4.2 & $0.2984 E-08$ & $0.1577 \mathrm{E}-08$ & $0.8880 E-09$ & $0.5654 E-09$ & $0.3859 \mathrm{E}-09$ \\
\hline 4.3 & $0.2937 E-08$ & $0.1551 \mathrm{E}-08$ & $0.8728 \mathrm{E}-09$ & $0.5554 \mathrm{E}-09$ & $0.3791 \mathrm{E}-09$ \\
\hline 4.4 & $0.2890 \mathrm{E}-08$ & $0.1526 \mathrm{E}-08$ & $0.8579 \varepsilon-09$ & $0.5457 \mathrm{E}-09$ & $0.3724 E-09$ \\
\hline 4.5 & $0.2843 E-08$ & $0.1501 E-08$ & $0.8432 E-09$ & $0.5361 \mathrm{E}-09$ & $0.3659 \mathrm{E}-09$ \\
\hline 4.6 & $0.2798 E-08$ & $0.1476 \mathrm{E}-08$ & $0.8289 \mathrm{E}-09$ & $0.5268 \mathrm{E}-09$ & $0.3595 E-09$ \\
\hline 4.7 & $0.2753 \mathrm{E}-08$ & $0.1452 \mathrm{E}-08$ & $0.8148 \mathrm{E}-09$ & $0.5176 \mathrm{E}-09$ & $0.3532 E-09$ \\
\hline 4.8 & $0.2709 \mathrm{E}-08$ & $0.1428 \mathrm{E}-08$ & $0.8010 \mathrm{E}-09$ & $0.5087 \mathrm{E}-09$ & $0.3471 E-09$ \\
\hline 4.9 & $0.2666 \mathrm{E}-08$ & $0.1405 E-08$ & $0.7875 E-09$ & $0.4999 \mathrm{E}-09$ & $0.3411 \mathrm{E}-09$ \\
\hline 5.0 & $0.2624 E-08$ & $0.1382 E-08$ & $0.7743 E-09$ & $0.4914 E-09$ & $0.3353 E-09$ \\
\hline
\end{tabular}


Table 11 e- $\mathrm{N}_{2}$ vibrational excitation rate coefficients, $\mathrm{cm}^{3} / \mathrm{s}$ $\left(v_{i}=8, J=50\right)$

\begin{tabular}{|c|c|c|c|c|c|}
\hline $\mathrm{r}, \mathrm{eV}$ & $v_{f}=9$ & 10 & 11 & 12 & 13 \\
\hline 0.1 & $0.3553 \mathrm{E}-11$ & $0.4482 \mathrm{E}-12$ & $0.5454 \mathrm{E}-13$ & $0.5137 \mathrm{E}-14$ & $0.5126 E-15$ \\
\hline 0.2 & $0.5640 E-10$ & $0.1865 E-10$ & $0.6094 E-11$ & $0.1798 \mathrm{E}-11$ & $0.5492 E-12$ \\
\hline 0.3 & $0.1795 E-09$ & $0.7939 \mathrm{E}-10$ & $0.3643 \mathrm{E}-10$ & $0.1607 \mathrm{E}-10$ & $0.7194 \mathrm{E}-11$ \\
\hline 0.4 & $0.3776 \mathrm{E}-09$ & $0.1879 \mathrm{E}-09$ & $0.9991 \mathrm{E}-10$ & $0.5300 E-10$ & $0.2827 E-10$ \\
\hline 0.5 & $0.6465 \mathrm{E}-09$ & $0.3392 E-09$ & $0.1927 \mathrm{E}-09$ & $0.1122 E-09$ & $0.6561 \mathrm{E}-10$ \\
\hline 0.6 & $0.9671 \mathrm{E}-09$ & $0.5202 E-09$ & $0.3045 E-09$ & $0.1861 E-09$ & $0.1148 E-09$ \\
\hline 0.7 & $0.1313 E-08$ & $0.7149 \mathrm{E}-09$ & $0.4238 \mathrm{E}-09$ & $0.2662 E-09$ & $0.1696 \mathrm{E}-09$ \\
\hline 0.8 & $0.1660 \mathrm{E}-08$ & $0.9092 \mathrm{E}-09$ & $0.5414 E-09$ & $0.3453 E-09$ & $0.2247 E-09$ \\
\hline 0.9 & $0.1991 E-08$ & $0.1093 \mathrm{E}-08$ & $0.6510 \mathrm{E}-09$ & $0.4190 E-09$ & $0.2766 \mathrm{E}-09$ \\
\hline 1.0 & $0.2293 E-08$ & $0.1260 E-08$ & $0.7493 E-09$ & $0.4847 E-09$ & $0.3231 \mathrm{E}-09$ \\
\hline 1.1 & $0.2561 E-08$ & $0.1407 \mathrm{E}-08$ & 0.8347 E-09 & $0.5414 E-09$ & $0.3635 E-09$ \\
\hline 1.2 & $0.2794 E-08$ & $0.1534 \mathrm{E}-08$ & 0.9072 & $0.5892 E-09$ & $0.3977 \mathrm{E}-09$ \\
\hline 1.3 & $0.2992 E-08$ & $0.1641 E-08$ & $0.9674 E-09$ & $0.6284 E-09$ & $0.4258 \mathrm{E}-09$ \\
\hline 1.4 & $0.3156 \varepsilon-08$ & $0.1730 \mathrm{E}-08$ & $0.1016 E-08$ & $0.6600 \mathrm{E}-09$ & $0.4485 E-09$ \\
\hline 1.5 & $0.3290 \mathrm{E}-08$ & $0.1801 E-08$ & $0.1055 E-08$ & $0.6847 E-09$ & $0.4664 \mathrm{E}-09$ \\
\hline 1.6 & $0.3397 E-08$ & $0.1858 \mathrm{E}-08$ & 0.108 & $0.7035 E-09$ & $0.4800 E-09$ \\
\hline 1.7 & $0.3480 \varepsilon-08$ & $0.1901 E-08$ & $0.1107 E-08$ & $0.7172 E-09$ & $0.4900 E-09$ \\
\hline 1.8 & $0.3543 E-08$ & $0.1933 E-08$ & $0.1123 E-08$ & $0.7266 E-09$ & $0.4969 \mathrm{E}-09$ \\
\hline 1.9 & $0.3587 \varepsilon-08$ & $0.1955 \mathrm{E}-08$ & $0.1133 E-08$ & $0.7322 \varepsilon-09$ & $0.5012 E-09$ \\
\hline 2.0 & $0.3616 E-08$ & $0.1968 \varepsilon-08$ & 0.113 & $0.7348 E-09$ & $0.5033 E-09$ \\
\hline 2.1 & $0.3631 E-08$ & $75 E-08$ & 0.113 & $0.7347 \varepsilon-09$ & $0.5035 \mathrm{E}-09$ \\
\hline 2.2 & $0.3635 E-08$ & $75 E-08$ & 0.1137 E- 08 & $0.7325 \mathrm{E}-09$ & $0.5022 \mathrm{E}-09$ \\
\hline 2.3 & $0.3630 \varepsilon-08$ & $0.1971 E-08$ & $0.1132 E-08$ & $0.7286 \mathrm{E}-09$ & $0.4996 \mathrm{E}-09$ \\
\hline 2.4 & $0.3617 \varepsilon-08$ & $0.1962 E-08$ & 0.112 & $0.7232 \mathrm{E}-09$ & $0.4960 \varepsilon-09$ \\
\hline 2.5 & $0.3597 \mathrm{E}-08$ & $0.1949 \mathrm{E}-08$ & 0.11 & $0.7166 \mathrm{E}-09$ & $16 \mathrm{E}-09$ \\
\hline 2.6 & $0.3571 \mathrm{E}-08$ & $0.1934 \mathrm{E}-08$ & $0.1105 E-08$ & $0.7090 E-09$ & $0.4864 E-09$ \\
\hline 2.7 & $0.3540 E-08$ & $0.1916 \mathrm{E}=08$ & $0.1093 E=08$ & $0.7007 E-09$ & $0.4808 \mathrm{E}-09$ \\
\hline 2.8 & $0.3506 \mathrm{E}-08$ & $0.1896 \mathrm{E}-08$ & $0.1080 \mathrm{E}-08$ & 0.691 & $6 \mathrm{E}-09$ \\
\hline 2.9 & $0.3469 E-08$ & $0.1874 \mathrm{E}-08$ & $0.1066 \mathrm{E}-08$ & $0.6823 \mathrm{E}-09$ & $0.4682 E-09$ \\
\hline 3.0 & $0.3429 \varepsilon-08$ & $0.1851 \mathrm{E}-08$ & $0.1052 E-08$ & $0.6725 E-09$ & $0.4615 E-09$ \\
\hline 3.1 & $0.3387 E-08$ & 0.18 & 0.1 & 0.66 & $0.4546 \mathrm{E}-09$ \\
\hline 3.2 & $0.3343 E-08$ & $0.1803 E-08$ & $0.1022 E-08$ & $0.6523 E-09$ & $0.4476 \mathrm{E}-09$ \\
\hline 3.3 & $E-08$ & 0.177 & 0.100 & 0.6 & 0.440 \\
\hline 3.4 & $0.3253 E-08$ & -08 & $0.9910 \mathrm{E}-09$ & $0.6316 \mathrm{E}-09$ & $0.4333 E-09$ \\
\hline 3.5 & $0.3206 \mathrm{E}-08$ & 0.1 & 0.9 & & $E-09$ \\
\hline 3.6 & $0.3160 \varepsilon-08$ & 0.17 & 0.95 & 0.6 & E -09 \\
\hline 3.7 & $0.3113 \varepsilon-08$ & $0.1674 E-08$ & $0.9442 E-09$ & $0.6006 \mathrm{E}-09$ & $0.4120 E-09$ \\
\hline 3.8 & $0.3066 \mathrm{E}-08$ & & & & $E-09$ \\
\hline 3.9 & $0.3020 \mathrm{E}-08$ & 0.162 & $0.9135 \mathrm{E}-09$ & $0.5803 E-09$ & $0.3980 E-09$ \\
\hline 4.0 & $0.2973 E-08$ & $0.1597 \mathrm{E}-08$ & $0.8983 \mathrm{E}-09$ & $0.5703 E-09$ & $0.3911 \mathrm{E}-09$ \\
\hline 4.1 & $0.2927 \mathrm{E}-08$ & $0.1572 E-08$ & $0.8834 \mathrm{E}-09$ & $0.5605 E-09$ & $0.3844 E-09$ \\
\hline 4.2 & $2 E-08$ & $E-08$ & $0.8687 E-09$ & $0.5509 E-09$ & $0.3777 E-09$ \\
\hline 4.3 & $0.2837 E-08$ & $0.1522 E-08$ & & & $0.3712 \mathrm{E}-09$ \\
\hline 4.4 & $0.2793 E-08$ & 0.1497 E- 08 & $0.8399 \mathrm{E}-09$ & $1 E-09$ & $0.3648 \mathrm{E}-09$ \\
\hline 4.5 & $0.2749 \mathrm{E}-08$ & $0.1473 E-08$ & $0.8259 \mathrm{E}-09$ & $0.5230 \mathrm{E}-09$ & $0.3585 \mathrm{E}-09$ \\
\hline 4.6 & $0.2706 \mathrm{E}-08$ & $0.1450 E-08$ & $0.8121 E-09$ & $0.5140 E-09$ & $0.3523 E-09$ \\
\hline 4.7 & $0.2663 \mathrm{E}-08$ & $0.1427 \mathrm{E}-08$ & $0.7986 \mathrm{E}-09$ & $0.5052 E-09$ & $0.3463 E-09$ \\
\hline 4.8 & $0.2622 E-08$ & $0.1404 E-08$ & $0.7854 E-09$ & $0.4966 \mathrm{E}-09$ & $0.3404 E-09$ \\
\hline 4. & & & & $0.4882 E-09$ & $0.3346 \mathrm{E}-09$ \\
\hline 5 & 0.2 & $0.1359 \mathrm{E}$ & 0.75 & $0.4800 E-09$ & $0.3289 \mathrm{E}-09$ \\
\hline
\end{tabular}


Table 12 e- $\mathrm{N}_{2}$ vibrational excitation rate coefficients, $\mathrm{cm}^{3} / \mathrm{s}$ $\left(v_{i}=9, J=50\right)$

\begin{tabular}{|c|c|c|c|c|c|}
\hline $\mathrm{eV}$ & $v_{f}=10$ & 11 & 12 & 13 & 14 \\
\hline 0.1 & $0.3501 \mathrm{E}-11$ & $0.4176 \mathrm{E}-12$ & $0.5071 \mathrm{E}-13$ & $0.4650 E-14$ & $0.4302 \mathrm{E}-15$ \\
\hline 0.2 & $0.4741 \mathrm{E}-10$ & $0.1489 \mathrm{E}-10$ & $0.5152 E-11$ & $0.1568 \mathrm{E}-11$ & $0.4814 \mathrm{E}-12$ \\
\hline 0.3 & $0.1535 \mathrm{E}-09$ & $0.6553 E-10$ & $0.3148 \mathrm{E}-10$ & $0.1444 \mathrm{E}-10$ & $0.6557 \mathrm{E}-11$ \\
\hline 0.4 & $0.3316 \mathrm{E}-09$ & $0.1612 \mathrm{E}-09$ & $0.8824 E-10$ & $0.4834 E-10$ & $0.2618 \mathrm{E}-10$ \\
\hline 0.5 & $0.5785 E-09$ & $0.2993 \mathrm{E}-09$ & $0.1730 \mathrm{E}-09$ & $0.1033 E-09$ & $0.6129 \mathrm{E}-10$ \\
\hline 0.6 & $0.8769 \mathrm{E}-09$ & $0.4679 \mathrm{E}-09$ & $0.2768 \mathrm{E}-09$ & $0.1726 \mathrm{E}-09$ & $0.1079 \mathrm{E}-09$ \\
\hline 0.7 & $0.1202 E-08$ & $0.6521 \mathrm{E}-09$ & $0.3890 \mathrm{E}-09$ & $0.2482 E-09$ & $0.1602 E-09$ \\
\hline 0.8 & $0.1532 E-08$ & $0.8381 E-09$ & $0.5008 \mathrm{E}-09$ & $0.3236 \mathrm{E}-09$ & $0.2131 \mathrm{E}-09$ \\
\hline 0.9 & $0.1847 \varepsilon-08$ & $0.1016 \mathrm{E}-08$ & $0.6062 \mathrm{E}-09$ & $0.3942 E-09$ & $0.2631 \mathrm{E}-09$ \\
\hline 1.0 & $0.2138 \mathrm{E}-08$ & $0.1179 \mathrm{E}-08$ & $0.7015 \mathrm{E}-09$ & $0.4577 \mathrm{E}-09$ & $0.3083 \mathrm{E}-09$ \\
\hline 1.1 & $0.2398 \mathrm{E}-08$ & $0.1324 \mathrm{E}-08$ & $0.7852 E-09$ & $0.5129 \mathrm{E}-09$ & $0.3477 \mathrm{E}-09$ \\
\hline 1.2 & $0.2625 E-08$ & $0.1450 \mathrm{E}-08$ & $0.8569 \mathrm{E}-09$ & $0.5597 \mathrm{E}-09$ & $0.3812 \mathrm{E}-09$ \\
\hline 1.3 & $0.2819 \mathrm{E}-08$ & $0.1557 \mathrm{E}-08$ & $0.9170 \mathrm{E}-09$ & $0.5985 \mathrm{E}-09$ & $0.4090 \mathrm{E}-09$ \\
\hline 1.4 & $0.2982 E-08$ & $0.1647 \varepsilon-08$ & $0.9663 E-09$ & $0.6299 \mathrm{E}-09$ & $0.4315 \mathrm{E}-09$ \\
\hline 1.5 & $0.3115 E-08$ & $0.1720 \mathrm{E}-08$ & $0.1006 \mathrm{E}-08$ & $0.6549 E-09$ & $0.4494 E-09$ \\
\hline 1.6 & $0.3223 E-08$ & $0.1778 \mathrm{E}-08$ & $0.1037 E-08$ & $0.6741 \mathrm{E}-09$ & $0.4632 E-09$ \\
\hline 1.7 & $0.3308 \varepsilon-08$ & $0.1824 \mathrm{E}-08$ & $0.1061 E-08$ & $0.6883 E-09$ & $0.4735 \mathrm{E}-09$ \\
\hline 1.8 & $0.3373 E-08$ & $0.1859 E-08$ & $0.1078 E-08$ & $0.6983 E-09$ & $0.4807 \mathrm{E}-09$ \\
\hline 1.9 & $0.3420 E-08$ & $0.1883 E-08$ & $0.1089 \mathrm{E}-08$ & $0.7047 \mathrm{E}-09$ & $0.4854 \mathrm{E}-09$ \\
\hline 2.0 & $0.3453 \mathrm{E}-08$ & $0.1900 \mathrm{E}-08$ & $0.1096 \mathrm{E}-08$ & $0.7081 \mathrm{E}-09$ & $0.4879 \mathrm{E}-09$ \\
\hline 2.1 & $0.3472 E-08$ & $0.1909 \mathrm{E}-08$ & $0.1099 \mathrm{E}-08$ & $0.7089 E-09$ & $0.4885 \mathrm{E}-09$ \\
\hline 2.2 & $0.3480 E-08$ & $0.1912 E-08$ & $0.1099 \mathrm{E}-08$ & $0.7076 E-09$ & $0.4877 \mathrm{E}-09$ \\
\hline 2.3 & $0.3478 E-08$ & $0.1910 \mathrm{E}-08$ & $0.1095 \mathrm{E}-08$ & $0.7044 \mathrm{E}-09$ & $0.4856 \mathrm{E}-09$ \\
\hline 2.4 & $0.3469 \mathrm{E}-08$ & $0.1904 \mathrm{E}-08$ & $0.1090 \mathrm{E}-08$ & $0.6999 E-09$ & $0.4824 \mathrm{E}-09$ \\
\hline 2.5 & $0.3453 E-08$ & $0.1894 \mathrm{E}-08$ & $0.1082 E-08$ & $0.6941 \mathrm{E}-09$ & $0.4784 \mathrm{E}-09$ \\
\hline 2.6 & $0.34318-08$ & $0.1880 \mathrm{E}-08$ & $0.1073 E-08$ & $0.6873 E-09$ & $0.4737 \mathrm{E}-09$ \\
\hline 2.7 & $0.3404 E-08$ & $0.1865 E-08$ & $0.1062 E-08$ & $0.6 ; 97 E-09$ & $0.4684 \mathrm{E}-09$ \\
\hline 2.8 & $0.3373 E-08$ & $0.1847 E-08$ & $0.1051 \mathrm{E}-08$ & $0.6715 \mathrm{E}-09$ & $0.4627 \mathrm{E}-09$ \\
\hline 2.9 & $0.3340 \mathrm{E}-08$ & $0.1828 \mathrm{E}-08$ & $0.1038 \mathrm{E}-08$ & $0.6628 \mathrm{E}-09$ & $0.4567 \mathrm{E}-09$ \\
\hline 3.0 & $0.3303 E-08$ & $0.1807 \mathrm{E}-08$ & $0.1025 E-08$ & $0.6537 E-09$ & $0.4503 \mathrm{E}-09$ \\
\hline 3.1 & $0.3265 E-08$ & $0.1785 \mathrm{E}-08$ & $0.1011 \mathrm{E}-08$ & $0.6444 \mathrm{E}-09$ & $0.4438 \mathrm{E}-09$ \\
\hline 3.2 & $0.3225 E-08$ & $0.1762 E-08$ & $0.9971 \mathrm{E}-09$ & $0.6348 \mathrm{E}-09$ & $0.4371 \mathrm{E}-09$ \\
\hline 3.3 & $0.3183 E-08$ & $0.1739 E-08$ & $0.9828 \mathrm{E}-09$ & $0.6251 \varepsilon-09$ & $0.4304 E-09$ \\
\hline 3.4 & $0.3141 \mathrm{E}-08$ & $0.1715 E-08$ & $0.9682 E-09$ & $0.6153 \varepsilon-09$ & $0.4236 \mathrm{E}-09$ \\
\hline 3.5 & $0.3097 \mathrm{E}-08$ & 0.16 & $0.9536 \mathrm{E}-09$ & $0.6055 E-09$ & $0.4167 \mathrm{E}-09$ \\
\hline 3.6 & $0.3054 E-08$ & $0.1666 \mathrm{E}-08$ & $0.9388 \mathrm{E}-09$ & 0.5957 E-09 & $0.4099 \mathrm{E}-09$ \\
\hline 3.7 & $0.3010 \mathrm{E}-08$ & $0.1641 \mathrm{E}-08$ & $0.9242 E-09$ & $0.5859 \mathrm{E}-09$ & $0.4031 \mathrm{E}-09$ \\
\hline 3.8 & $0.2966 \mathrm{E}-08$ & $0.1617 \mathrm{E}-08$ & $0.9095 E-09$ & $0.5762 E-09$ & $0.3964 E-09$ \\
\hline 3.9 & $0.2922 E-08$ & $0.1592 E-08$ & $0.8950 \mathrm{E}-09$ & $0.5666 \mathrm{E}-09$ & $0.3897 \mathrm{E}-09$ \\
\hline 4.0 & $0.2878 \mathrm{E}-08$ & $0.1568 \mathrm{E}-08$ & $0.8806 \mathrm{E}-09$ & $0.5571 \varepsilon-09$ & $0.3831 \mathrm{E}-09$ \\
\hline 4.1 & $0.2835 \mathrm{E}-08$ & $0.1544 E-08$ & $0.8663 E-09$ & $0.5477 \varepsilon-09$ & $0.3766 \mathrm{E}-09$ \\
\hline 4.2 & $0.2792 E-08$ & $0.1520 \mathrm{E}-08$ & $0.8522 E-09$ & $0.5384 E-09$ & $0.3701 E-09$ \\
\hline 4.3 & $0.27498-08$ & $0.1496 \mathrm{E}-08$ & $0.8384 \mathrm{E}-09$ & $0.5293 E-09$ & $0.3638 \mathrm{E}-09$ \\
\hline 4.4 & $0.2707 \mathrm{E}-08$ & $0.1473 E-08$ & $0.8247 E-09$ & $0.5204 \mathrm{E}-09$ & $0.3576 \mathrm{E}-09$ \\
\hline 4.5 & $0.2665 E-08$ & $0.1449 E-08$ & $0.8112 E-09$ & $0.5116 E-09$ & $0.3515 \mathrm{E}-09$ \\
\hline 4.6 & $0.2624 E-08$ & $0.1427 \varepsilon-08$ & $0.7980 \mathrm{E}-09$ & $0.5030 \mathrm{E}-09$ & $0.3456 \mathrm{E}-09$ \\
\hline 4.7 & $0.2584 E-08$ & $0.1404 E-08$ & $0.7850 \mathrm{E}-09$ & $0.4946 \mathrm{E}-09$ & $0.3397 E-09$ \\
\hline 4.8 & $0.2544 \mathrm{E}-08$ & $0.1382 E-08$ & $0.7723 \mathrm{E}-09$ & $0.4863 \mathrm{E}-09$ & $0.3340 E-09$ \\
\hline 4.9 & $0.2505 \mathrm{E}-08$ & $0.1361 \mathrm{E}-\mathrm{C}$ & $0.7598 \mathrm{E}-09$ & $0.4782 E-09$ & $0.3284 E-09$ \\
\hline 5.0 & $0.2466 \mathrm{E}-08$ & $0.1340 \mathrm{E}-08$ & $0.7475 E-09$ & $0.4703 E-09$ & $0.3229 E-09$ \\
\hline
\end{tabular}


Table 13 e- $\mathrm{N}_{2}$ vibrational excitation rate coefficients, $\mathrm{cm}^{3} / \mathrm{s}$ $\left(v_{i}=10, J=50\right)$

\begin{tabular}{|c|c|c|c|c|c|}
\hline$T, e V$ & $v_{f}=11$ & 12 & 13 & 14 & 15 \\
\hline 0.1 & $0.3113 \mathrm{E}-11$ & $0.3620 \mathrm{E}-12$ & $0.4202 E-13$ & $0.4023 \mathrm{E}-14$ & $0.3604 \mathrm{E}-15$ \\
\hline 0.2 & $0.3937 \mathrm{E}-10$ & $0.1221 \mathrm{E}-10$ & $0.4243 \mathrm{E}-11$ & $0.1404 \mathrm{E}-11$ & $0.4495 \mathrm{E}-12$ \\
\hline 0.3 & $0.1327 \mathrm{E}-09$ & $0.5657 \varepsilon-10$ & $0.2722 E-10$ & $0.1320 \mathrm{E}-10$ & $0.6207 E-11$ \\
\hline 0.4 & $0.2946 \mathrm{E}-09$ & $0.1438 \mathrm{E}-09$ & $0.7850 \mathrm{E}-10$ & $0.4460 \mathrm{E}-10$ & $0.2483 \mathrm{E}-10$ \\
\hline 0.5 & $0.5231 E-09$ & $0.2723 \mathrm{E}-09$ & $0.1567 \mathrm{E}-09$ & $0.9586 E-10$ & $0.5823 \mathrm{E}-10$ \\
\hline 0.6 & $0.8023 E-09$ & $0.4314 \mathrm{E}-09$ & $0.2538 \mathrm{E}-09$ & $0.1611 \mathrm{E}-09$ & $0.1027 \mathrm{E}-09$ \\
\hline 0.7 & $0.1109 \mathrm{E}-08$ & $0.6071 E-09$ & 0.3601 E- 09 & $0.2327 \mathrm{E}-09$ & $0.1529 \mathrm{E}-09$ \\
\hline 0.8 & $0.1422 \mathrm{E}-08$ & 0.786 IE-09 & $0.4671 \mathrm{E}-09$ & $0.3046 \mathrm{E}-09$ & $0.2038 \mathrm{E}-09$ \\
\hline 0.9 & $0.1725 E-08$ & $0.9585 E-09$ & $0.5689 \mathrm{E}-09$ & $0.3725 \mathrm{E}-09$ & $0.2522 E-09$ \\
\hline 1.0 & $0.2005 E-08$ & $0.1118 \mathrm{E}-08$ & $0.6617 \mathrm{E}-09$ & $0.4338 \mathrm{E}-09$ & $0.2961 \mathrm{E}-09$ \\
\hline 1.1 & $0.2256 \mathrm{E}-08$ & $0.1261 E-08$ & $0.7439 \mathrm{E}-09$ & $0.4876 \mathrm{E}-09$ & $0.3346 \mathrm{E}-09$ \\
\hline 1.2 & $0.2477 E-08$ & $0.1386 E-08$ & $0.8148 \mathrm{E}-09$ & $0.5334 \mathrm{E}-09$ & $0.3674 E-09$ \\
\hline 1.3 & $0.2667 \mathrm{E}-08$ & $0.1492 \mathrm{E}-08$ & $0.8748 \mathrm{E}-09$ & $0.5717 \mathrm{E}-09$ & 0.39 \\
\hline 1.4 & $0.2827 \mathrm{E}-08$ & $0.1582 E-08$ & $0.9245 \mathrm{E}-09$ & 0.603 & 0.41 \\
\hline 1.5 & $0.2960 \mathrm{E}-08$ & $0.1657 \mathrm{E}-08$ & $0.9648 \mathrm{E}-09$ & $0.6280 \mathrm{E}-09$ & 0.43 \\
\hline 1.6 & $0.3068 \mathrm{E}-08$ & $0.1717 \mathrm{E}-08$ & $0.9969 \mathrm{E}-09$ & $0.6475 E-09$ & $0.4488 \mathrm{E}-09$ \\
\hline 1.7 & $0.3154 E-08$ & $0.1764 E-08$ & $0.1022 E-08$ & $0.6622 \mathrm{E}-09$ & $0.4592 E-09$ \\
\hline 1.8 & $0.3221 \mathrm{E}-08$ & $0.1801 E-08$ & $0.1040 \mathrm{E}-08$ & 0.6727 E-09 & 0.46 \\
\hline 1.9 & 0.327 & 0.1827 & 0.105 & 0.67 & 0.471 \\
\hline 2.0 & $0.3305 \mathrm{E}$ & $0.1846 \mathrm{E}-08$ & $0.1061 \mathrm{E}-08$ & 0.683 & $0.4744 E-09$ \\
\hline 2.1 & $0.3326 \mathrm{E}-08$ & $0.1857 E-08$ & $0.1066 \mathrm{E}-08$ & $0.6853 E-09$ & 0.47 \\
\hline 2.2 & 0.333 & 0.186 & $0.1066 \mathrm{E}-08$ & 0.684 & $E-09$ \\
\hline 2.3 & $0.3339 \mathrm{E}-08$ & $0.1862 E-08$ & $0.1065 \mathrm{E}-08$ & 0.6 & 0.47 \\
\hline 2.4 & $0.3333 E-08$ & $0.1858 \mathrm{E}-08$ & $0.1060 \mathrm{E}-08$ & $0.6784 \mathrm{E}-09$ & 0.47 \\
\hline 2.5 & $0.3320 \mathrm{E}$ & $0.1850 E-08$ & $0.1054 E-08$ & $0.6734 \mathrm{E}-09$ & $0.4667 E-09$ \\
\hline 2.6 & $0.3301 E-08$ & $0.1839 E-08$ & 0.104 & $0.66^{\circ}$ & $0.4624 E-09$ \\
\hline 2.7 & $0.3278 E-08$ & $0.1825 \mathrm{E}-08$ & 0.10 & $0.6604 \mathrm{E}-09$ & $0.4575 E-09$ \\
\hline 2.8 & $0.3250 \mathrm{E}-08$ & $0.1809 \mathrm{E}-08$ & 0.102 & $0.6529 \mathrm{E}-09$ & $0.4521 \mathrm{E}-09$ \\
\hline 2.9 & $0.3220 \mathrm{E}-08$ & 0.179 & 0.10 & 0.64 & $E-09$ \\
\hline 3.0 & $0.3186 \mathrm{E}-08$ & 0.177 & 0.100 & $E-09$ & $E-09$ \\
\hline 3.1 & $0.3151 \mathrm{E}-08$ & 0.175 & 0.980 & 0. & $E-09$ \\
\hline 3.2 & $0.3114 \mathrm{E}-08$ & 0.1 & $E-09$ & $86 E-09$ & $E-09$ \\
\hline 3 & $0.3075 E-08$ & $0.1708 \mathrm{E}-08$ & $0.9632 \mathrm{E}-09$ & $0.6094 \mathrm{E}-09$ & $0.4214 E-09$ \\
\hline .4 & 0.3035 & 0.16 & 0.94 & 0.6 & 0.4 \\
\hline 3 & $0.2995 \mathrm{E}-08$ & $0.1662 E-08$ & $\varepsilon-09$ & $E-09$ & $E-09$ \\
\hline & $0.2954 E-08$ & $0.1639 \mathrm{E}-08$ & $0.9217 \mathrm{E}-09$ & & E-09 \\
\hline 37 & $0.2913 E-08$ & $0.1616 \mathrm{E}-\mathrm{C}$ & 0.9 & 0.5 & 0. \\
\hline 3 & $0.2871 \mathrm{E}-0$ & $0.1592 \mathrm{E}-08$ & $0.8937 \mathrm{E}-09$ & $0.5629 \mathrm{E}-09$ & $0.3886 \mathrm{E}-09$ \\
\hline & 0.28 & 0.156 & & & \\
\hline 4.0 & $0.2788 \mathrm{E}-0$ & 0.1 & 0.8 & $E-09$ & $0.3758 \mathrm{E}-09$ \\
\hline 4.1 & $0.2747 \mathrm{E}-08$ & $0.1522 \mathrm{E}-08$ & $0.8524 E-09$ & $0.5357 \mathrm{E}-09$ & $0.3695 \mathrm{E}-09$ \\
\hline 4.2 & $0.2706 \mathrm{E}-08$ & 0.14 & 0.838 & $0.5268 \mathrm{E}-09$ & $0.3633 E-09$ \\
\hline 4.3 & $0.2665 E-08$ & $E-08$ & 0.82 & $0.5181 E-09$ & $0.3572 E-09$ \\
\hline 4.4 & 0.262 & 0.145 & $E-09$ & & $0.3512 E-09$ \\
\hline 4.5 & $0.2585 \mathrm{E}-08$ & $0.1431 \mathrm{E}-\mathrm{C}$ & 0.79 & $0.5010 \mathrm{E}-09$ & $0.3453 E-09$ \\
\hline 4.6 & $0.2546 \mathrm{E}-0$ & $0.1409 \mathrm{E}-08$ & $0.7866 \mathrm{E}-09$ & $0.4927 \mathrm{E}-09$ & $0.3395 \mathrm{E}-09$ \\
\hline 4.7 & $0.2508 \mathrm{E}-08$ & $0.1387 \mathrm{E}-08$ & $0.7741 \mathrm{E}-09$ & $0.4846 \mathrm{E}-09$ & $0.3338 \mathrm{E}-09$ \\
\hline 4.8 & & 0. & 0.7 & $6 E-09$ & $0.3282 \mathrm{E}-09$ \\
\hline 4. & $0.2432 \mathrm{E}-$ & 0.13 & $0.7497 \mathrm{E}-$ & $0.4687 \mathrm{E}-09$ & $0.3227 \mathrm{E}-09$ \\
\hline & $0.2395 \mathrm{E}-\mathrm{C}$ & $0.1324 E-08$ & $0.7378 \mathrm{E}-09$ & $0.4611 \mathrm{E}-09$ & $0.3174 E-09$ \\
\hline
\end{tabular}


Table 14 e- $\mathrm{N}_{2}$ vibrational excitation rate coefficients, $\mathrm{cm}^{3} / \mathrm{s}$ $\left(v_{i}=11, \mathrm{~J}=50\right)$

\begin{tabular}{|c|c|c|c|c|c|}
\hline $\mathrm{T}, \mathrm{eV}$ & $v_{f}=12$ & 13 & 14 & 15 & 16 \\
\hline 0.1 & $0.2509 \mathrm{E}-11$ & $0.2969 \mathrm{E}-12$ & $0.3252 \varepsilon-13$ & $0.3352 E-14$ & $0.3285 E-15$ \\
\hline 0.2 & $0.3216 \mathrm{E}-10$ & $0.1045 E-10$ & $0.3597 \varepsilon-11$ & $0.1275 \mathrm{E}-11$ & $0.4375 \varepsilon-12$ \\
\hline 0.3 & $0.1148 \mathrm{E}-09$ & $0.5063 E-10$ & $0.2422 E-10$ & $0.1219 \mathrm{E}-10$ & $0.5959 \mathrm{E}-11$ \\
\hline 0.4 & $0.2631 \mathrm{E}-09$ & $0.1314 \mathrm{E}-09$ & $0.7136 \mathrm{E}-10$ & $0.4143 \mathrm{E}-10$ & $0.2369 \mathrm{E}-10$ \\
\hline 0.5 & $0.4757 \mathrm{E}-09$ & $0.2520 E-09$ & $0.1443 \mathrm{E}-09$ & $0.8950 E-10$ & $0.5546 \mathrm{E}-10$ \\
\hline 0.6 & $0.7381 E-09$ & $0.4029 E-09$ & $0.2358 E-09$ & $0.1511 \mathrm{E}-09$ & $0.9791 \mathrm{E}-10$ \\
\hline 0.7 & $0.1029 \mathrm{E}-08$ & $0.5709 E-09$ & $0.3369 \mathrm{E}-09$ & $0.2192 \mathrm{E}-09$ & $0.1459 \mathrm{E}-09$ \\
\hline 0.8 & $0.1327 \varepsilon-08$ & $0.7434 \mathrm{E}-09$ & $0.4396 \mathrm{E}-09$ & $0.2880 \mathrm{E}-09$ & $0.1948 \mathrm{E}-09$ \\
\hline 0.9 & $0.1617 \varepsilon-08$ & $0.9106 \mathrm{E}-09$ & $0.5379 \varepsilon-09$ & $0.3533 \varepsilon-09$ & $0.2415 E-09$ \\
\hline 1.0 & $0.1887 E-08$ & $0.1066 E-08$ & $0.6283 E-09$ & $0.4126 \mathrm{E}-09$ & $0.2840 \mathrm{E}-09$ \\
\hline 1.1 & $0.2131 \varepsilon-08$ & $0.1206 \mathrm{E}-08$ & $0.7089 \mathrm{E}-09$ & $0.4649 \mathrm{E}-09$ & $0.3214 E-09$ \\
\hline 1.2 & $0.2346 \mathrm{E}-08$ & $0.1330 \mathrm{E}-08$ & $0.77898-09$ & $0.5098 E-09$ & $0.3534 E-09$ \\
\hline 1.3 & $0.2531 \mathrm{E}-08$ & $0.1436 E-08$ & $0.8385 \varepsilon-09$ & $0.5475 \mathrm{E}-09$ & $0.3803 \varepsilon-09$ \\
\hline 1.4 & $0.2689 \mathrm{E}-08$ & $0.1526 \mathrm{E}-08$ & $0.8883 \varepsilon-09$ & $0.5786 \mathrm{E}-09$ & $0.4023 E-09$ \\
\hline 1.5 & $0.2820 \mathrm{E}-08$ & $0.1601 E-08$ & $0.9291 \varepsilon-09$ & $0.6036 \mathrm{E}-09$ & $0.4199 E-09$ \\
\hline 1.6 & $0.2928 \mathrm{E}-08$ & $0.1662 E-08$ & $0.9619 \varepsilon-09$ & $0.6233 E-09$ & $0.4338 \varepsilon-09$ \\
\hline 1.7 & $0.3014 E-08$ & $0.1710 \mathrm{E}-08$ & $0.9875 E-09$ & $0.6383 \mathrm{E}-09$ & $0.4442 E-09$ \\
\hline 1.8 & $0.3081 \varepsilon-08$ & $0.1748 \mathrm{E}-08$ & $0.1007 \varepsilon-08$ & $0.6494 \mathrm{E}-09$ & $0.4519 \mathrm{E}-09$ \\
\hline 1.9 & $0.3132 \varepsilon-08$ & $0.1777 \mathrm{E}-08$ & $0.1021 \varepsilon-08$ & $0.6569 \mathrm{E}-09$ & $0.4570 E-09$ \\
\hline 2.0 & $0.3169 \mathrm{E}-08$ & $0.1797 \mathrm{E}-08$ & $0.1030 E-08$ & $0.6615 \varepsilon-09$ & $0.4600 E-09$ \\
\hline 2.1 & $0.3193 E-08$ & $0.1810 E-08$ & $0.1036 \mathrm{E}-08$ & $0.6636 \mathrm{E}-09$ & $0.4613 \varepsilon-09$ \\
\hline 2.2 & $0.3206 \mathrm{E}-08$ & $0.1817 E-08$ & $0.1038 \mathrm{E}-08$ & $0.6637 \mathrm{E}-09$ & $0.4611 \mathrm{E}-09$ \\
\hline 2.3 & $0.3211 E-08$ & $0.1819 E-08$ & $0.1037 E-08$ & $0.6619 E-09$ & $0.4597 \varepsilon-09$ \\
\hline 2.4 & $0.3207 \varepsilon-08$ & $0.1816 E-08$ & $0.1034 E-08$ & $0.6587 \mathrm{E}-09$ & $0.4573 \mathrm{E}-09$ \\
\hline 2.5 & $0.3197 \varepsilon-08$ & $0.1810 E-08$ & $0.1029 E-08$ & $0.6543 E-09$ & $0.4540 \varepsilon-09$ \\
\hline 2.6 & $0.3181 E-08$ & $0.1800 \mathrm{E}-08$ & 0.10 & EE-09 & $0.4500 \mathrm{E}-09$ \\
\hline 2.7 & $0.3160 \varepsilon-08$ & $0.1788 \mathrm{E}-08$ & $0.1013 E-08$ & $0.6426 \mathrm{E}-09$ & $0.4454 \mathrm{E}-09$ \\
\hline 2.8 & $0.3136 \mathrm{E}-08$ & $0.1773 E-08$ & $0.1004 E-08$ & $0.6356 \mathrm{E}-09$ & $0.4404 E-09$ \\
\hline 2.9 & $0.3108 \mathrm{E}-08$ & $0.1757 E-08$ & $0.9933 E-09$ & $0.6282 E-09$ & $0.4350 E-09$ \\
\hline 3.0 & $0.3077 \mathrm{E}-08$ & $0.1739 \mathrm{E}-08$ & $0.9821 \mathrm{E}-09$ & $0.6203 E-09$ & $0.4293 \mathrm{E}-09$ \\
\hline 3.1 & $0.3044 \mathrm{E}-08$ & $0.1720 \mathrm{E}-08$ & $0.9702 E-09$ & 0.612 & $0.4234 E-09$ \\
\hline 3.2 & $0.3009 \mathrm{E}-08$ & $0.1700 \mathrm{E}-08$ & $0.9579 \mathrm{E}-09$ & $0.6035 E-09$ & $0.4173 E-09$ \\
\hline 3.3 & $0.2973 E-08$ & $0.1679 \mathrm{E}-08$ & $0.9452 E-09$ & $0.5949 \mathrm{E}-09$ & $0.4112 \mathrm{E}-09$ \\
\hline 3.4 & $0.2936 \mathrm{E}-08$ & $0.1657 \varepsilon-08$ & 0.932 & $0.5861 \mathrm{E}-09$ & $E-09$ \\
\hline 3.5 & $0.2898 E-08$ & $0.1635 \mathrm{E}-08$ & $0.91918-09$ & $0.5772 E-09$ & $0.3986 \mathrm{E}-09$ \\
\hline 3.6 & $0.2859 \varepsilon-08$ & $0.1613 E-08$ & & & \\
\hline 3.7 & $0.2820 E-08$ & -08 & $0.8925 \mathrm{E}-09$ & $0.5595 \mathrm{E}-09$ & $0.3860 \mathrm{E}-09$ \\
\hline 3.8 & $0.2781 E-08$ & $0.1568 E-08$ & $0.8791 \mathrm{E}-09$ & $0.5506 \mathrm{E}-09$ & $0.3798 \mathrm{E}-09$ \\
\hline 3.9 & $0.2742 E-08$ & $0.1546 \mathrm{E}-08$ & $0.8658 \mathrm{E}-09$ & $0.5418 E-09$ & $0.3736 \mathrm{E}-09$ \\
\hline 4.0 & $0.2702 E-08$ & $0.1523 E-08$ & $0.8526 \mathrm{E}-09$ & $0.5331 E-09$ & $0.3674 E-09$ \\
\hline 4.1 & $0.2663 \mathrm{E}-08$ & 0.150 & $0.8394 E-09$ & $0.5244 E-09$ & $0.3613 \mathrm{E}-09$ \\
\hline 4.2 & $0.2624 E-08$ & $0.1478 E-08$ & $0.8264 E-09$ & $9 E-09$ & $0.3553 \mathrm{E}-09$ \\
\hline 4.3 & $0.2585 E-08$ & $0.1456 \mathrm{E}-08$ & $0.8135 E-09$ & $0.5075 \mathrm{E}-09$ & $0.3494 \mathrm{E}-09$ \\
\hline 4.4 & $0.2547 \varepsilon-08$ & $0.1434 E-08$ & $0.8008 \mathrm{E}-09$ & $0.4992 \mathrm{E}-09$ & $0.3436 \mathrm{E}-09$ \\
\hline 4.5 & $0.2509 \mathrm{E}-08$ & $0.1413 E-08$ & $0.7883 E-09$ & $0.4911 \mathrm{E}-09$ & $0.3379 E-09$ \\
\hline 4.6 & $0.2472 E-08$ & $0.1391 E-08$ & $0.7759 \varepsilon-09$ & $0.4831 E-09$ & $0.3323 \mathrm{E}-09$ \\
\hline 4.7 & $0.2435 E-08$ & $0.1370 \mathrm{E}-08$ & $0.7638 \mathrm{E}-09$ & $0.4752 E-09$ & $0.3268 \mathrm{E}-09$ \\
\hline 4.8 & $0.2398 \mathrm{E}-08$ & $0.1349 E-08$ & $0.7518 \varepsilon-09$ & $0.4675 E-09$ & $0.3214 \mathrm{E}-09$ \\
\hline 4.9 & $0.2362 \mathrm{E}-\mathrm{C}$ & 0.132 & $1 E-09$ & $0.4599 \mathrm{E}-09$ & $0.3161 \mathrm{E}-09$ \\
\hline 5.0 & $0.2327 \mathrm{E}-0$ & $0.1309 \mathrm{E}-08$ & $0.7285 E-09$ & $0.4525 \mathrm{E}-09$ & $0.3109 \mathrm{E}-09$ \\
\hline
\end{tabular}


Table 15 e-N 2 vibrational excitation rate coefficients, $\mathrm{cm}^{3} / \mathrm{s}$ $\left(v_{j}=12, J=50\right)$

\begin{tabular}{|c|c|c|c|c|c|}
\hline $\mathrm{T}, \mathrm{eV}$ & $v_{f}=13$ & 14 & 15 & 16 & 17 \\
\hline 0.1 & $0.1885 \mathrm{E}-11$ & $0.2339 \mathrm{E}-12$ & $0.2545 E-13$ & $0.2872 E-14$ & $0.3400 \mathrm{E}-15$ \\
\hline 0.2 & $0.2669 \mathrm{E}-10$ & $0.9168 \mathrm{E}-11$ & 0.324 & $0.1190 E-11$ & $0.4338 \mathrm{E}-12$ \\
\hline 0.3 & $0.1012 E-09$ & $0.4597 \mathrm{E}-10$ & $0.2232 E-10$ & $0.1139 \mathrm{E}-10$ & $0.5754 \mathrm{E}-11$ \\
\hline 0.4 & $0.2383 E-09$ & $0.1212 E-09$ & $0.6629 E-10$ & & \\
\hline 0.5 & $0.4375 E-09$ & $0.2348 \mathrm{E}-09$ & $0.1349 \mathrm{E}-09$ & $0.8404 E-10$ & $0.5301 \mathrm{E}-10$ \\
\hline 0.6 & $0.6854 E-09$ & $0.3784 E-09$ & $0.2217 \mathrm{E}-09$ & $0.1423 E-09$ & $0.9359 \mathrm{E}-10$ \\
\hline 0.7 & $0.9620 E-09$ & $0.5396 \mathrm{E}-09$ & $0.3185 E-09$ & $0.2071 \mathrm{E}-09$ & $0.1396 \mathrm{E}-09$ \\
\hline 0.8 & $0.1247 \mathrm{E}-08$ & $0.7062 E-09$ & $0.4174 \mathrm{E}-09$ & $0.2730 \mathrm{E}-09$ & $0.1867 \mathrm{E}-09$ \\
\hline 0.9 & $0.1525 E-08$ & $0.8687 E-09$ & $0.5128 E-09$ & $0.3358 \mathrm{E}-09$ & 0.2 \\
\hline 1.0 & $0.1786 E-08$ & $0.1021 E-08$ & $0.6011 E-09$ & $0.3933 E-09$ & $0.2730 \mathrm{E}-09$ \\
\hline 1.1 & $0.2022 E-08$ & $0.1158 E-08$ & $0.6802 E-09$ & $0.4441 E-09$ & $0.3094 \mathrm{E}-09$ \\
\hline 1.2 & $0.2231 E-08$ & $0.1280 E-08$ & $0.7494 E-09$ & $0.4880 E-09$ & $0.3406 \mathrm{E}-09$ \\
\hline 1.3 & $0.2413 E-08$ & $0.1386 \mathrm{E}-08$ & $0.8086 \mathrm{E}-09$ & $0.5251 E-09$ & $0.3670 \mathrm{E}-09$ \\
\hline 1.4 & $0.2567 E-08$ & $.1476 \mathrm{E}-08$ & $0.8585 E-09$ & $0.5558 \mathrm{E}-09$ & 0.38 \\
\hline 1.5 & $0.2697 \varepsilon-08$ & $0.1551 \mathrm{E}-08$ & $0.8996 E-09$ & $0.5807 \varepsilon-09$ & $0.4061 \mathrm{E}-09$ \\
\hline 1.6 & $0.2804 E-08$ & 0.1612 & 0.9329 & $E-09$ & 0.419 \\
\hline 1.7 & $0.2890 \varepsilon-08$ & 0.1662 & $0.9593 E-09$ & $0.6158 \mathrm{E}-09$ & 0.43 \\
\hline 1.8 & $0.2958 \mathrm{E}-08$ & $0.1701 \mathrm{E}-08$ & $0.9795 E-09$ & $0.6272 E-09$ & $0.4381 E-09$ \\
\hline 1.9 & 0.30 & -08 & -09 & $0.6351 E-09$ & $E-09$ \\
\hline 2.0 & $0.3048 E-08$ & 8 & 0.1 & 0.640 & $E-09$ \\
\hline 2.1 & $0.3074 E-08$ & 0.1767 & 0.1 & 0.642 & 0.44 \\
\hline 2.2 & $0.3089 \varepsilon$ & 0177 & 0.101 & $0.6435 E-09$ & $0.4484 \mathrm{E}-09$ \\
\hline 2.3 & 0.30 & $0.177 \varepsilon$ & -08 & $0.6423 E-09$ & 0.44 \\
\hline 2.4 & 0.30 & -08 & 0.1 & $0.6396 \mathrm{E}-09$ & $E-09$ \\
\hline 2.5 & 0.30 & .177 & 0. & -09 & $E-09$ \\
\hline 2.6 & $0.3072 \varepsilon-08$ & $.1764 \mathrm{E}-08$ & $0.1002 E-08$ & $0.6309 \mathrm{E}-09$ & $0.4384 E-09$ \\
\hline 2.7 & $0.3054 E-08$ & $0.1753 E-08$ & $0.9943 E-09$ & $0.6252 E-09$ & $0.4341 \mathrm{E}-09$ \\
\hline 2.8 & $0.3031 E-08$ & .1 & 0.9 & $188 E-09$ & 0.4 \\
\hline 2.9 & $0.3006 \mathrm{E}-08$ & .08 & 0.976 & $0.6118 \mathrm{E}-09$ & E-09 \\
\hline 3.0 & $0.2978 E-08$ & 0 & 0.9655 & $0.6045 \mathrm{E}-09$ & $E-09$ \\
\hline 3.1 & 0.29 & 8 & 9 & $E-09$ & 0.4 \\
\hline 3.2 & $0.2915 E-08$ & 0.1671 & -09 & $0.5887 \mathrm{E}-09$ & $E-09$ \\
\hline S. & 0.28 & 0.165 & & 0.580 & 0.4 \\
\hline 3.4 & $0.2846 E-08$ & .163 & 0.9 & $E-09$ & $E-09$ \\
\hline 3.5 & $0.2810 E-08$ & 0.16 & & E-09 & E-09 \\
\hline 36 & 0.27 & 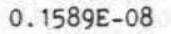 & & -09 & $0.3836 \mathrm{E}-09$ \\
\hline 3.7 & $0.2736 E-08$ & $.1567 \mathrm{E}-08$ & & E-09 & E-09 \\
\hline 3 & & & & & E-09 \\
\hline 3.9 & $0.2661 E-08$ & .15 & 0.8 & -09 & 0.3 \\
\hline 4.0 & $0.2624 E-08$ & 0.15 & & $0.5215 E-09$ & $0.3595 \mathrm{E}-09$ \\
\hline 4. & 0.25 & & & E-09 & $0.3537 \mathrm{E}-09$ \\
\hline 4.2 & 0.25 & & 0.1 & $E-09$ & E-09 \\
\hline 4.3 & $0.2512 E-08$ & $0.1437 \varepsilon-$ & & E-09 & E- 09 \\
\hline 4.4 & $0.2475 E-08$ & 0.1 & 0.79 & $0.4889 \mathrm{E}-09$ & $0.3365 \mathrm{E}-09$ \\
\hline 4.5 & $0.2439 \mathrm{E}-08$ & $0.1395 E-08$ & $0.7796 E-09$ & $0.4811 \mathrm{E}-09$ & $0.3310 \mathrm{E}-09$ \\
\hline 4.6 & & & $6 E-09$ & & $5 E-09$ \\
\hline 4.7 & $0.2367 \varepsilon-0$ & & $0.7558 \mathrm{E}-09$ & $0.4657 E-09$ & $0.3202 \mathrm{E}-09$ \\
\hline 4.8 & $0.2332 E-$ & $0.1333 \mathrm{E}$ & $1 \mathrm{E}-09$ & $0.4583 \mathrm{E}-09$ & $0.3149 \mathrm{E}-09$ \\
\hline 4. & & & & & \\
\hline E & $0=$ & 0. & $0.7214 \mathrm{E}-09$ & $0.4437 \mathrm{E}-09$ & $0.3048 \mathrm{E}-09$ \\
\hline
\end{tabular}


Table 16 e-N 2 vibrational de-excitation rate coefficients, $\mathrm{cm}^{3} / \mathrm{s}$ $\left(v_{1}=1, J=50\right)$

\begin{tabular}{cccc}
\hline $\mathrm{T}, \mathrm{eV}$ & $\mathrm{v}_{\mathrm{f}}=0$ & $\mathrm{~T}, \mathrm{eV}$ & $\mathrm{v}_{\mathrm{f}}=0$ \\
\hline 0.1 & $0.2783 \mathrm{E}-12$ & 2.6 & $0.7801 \mathrm{E}-08$ \\
0.2 & $0.8537 \mathrm{E}-10$ & 2.7 & $0.7604 \mathrm{E}-08$ \\
0.3 & $0.7357 \mathrm{E}-09$ & 2.8 & $0.7411 \mathrm{E}-08$ \\
0.4 & $0.2070 \mathrm{E}-08$ & 2.9 & $0.7223 \mathrm{E}-08$ \\
0.5 & $0.3695 \mathrm{E}-08$ & 3.0 & $0.7039 \mathrm{E}-08$ \\
0.6 & $0.5263 \mathrm{E}-08$ & 3.1 & $0.6862 \mathrm{E}-08$ \\
0.7 & $0.6602 \mathrm{E}-08$ & 3.2 & $0.6689 \mathrm{E}-08$ \\
0.8 & $0.7663 \mathrm{E}-08$ & 3.3 & $0.6522 \mathrm{E}-08$ \\
0.9 & $0.8461 \mathrm{E}-08$ & 3.4 & $0.6360 \mathrm{E}-08$ \\
1.0 & $0.9030 \mathrm{E}-08$ & 3.5 & $0.6203 \mathrm{E}-08$ \\
1.1 & $0.9413 \mathrm{E}-08$ & 3.6 & $0.6052 \mathrm{E}-08$ \\
1.2 & $0.9646 \mathrm{E}-08$ & 3.7 & $0.5905 \mathrm{E}-08$ \\
1.3 & $0.9764 \mathrm{E}-08$ & 3.8 & $0.5764 \mathrm{E}-08$ \\
1.4 & $0.9793 \mathrm{E}-08$ & 3.9 & $0.5627 \mathrm{E}-08$ \\
1.5 & $0.9754 \mathrm{E}-08$ & 4.0 & $0.5495 \mathrm{E}-08$ \\
1.6 & $0.9663 \mathrm{E}-08$ & 4.1 & $0.5367 \mathrm{E}-08$ \\
1.7 & $0.9535 \mathrm{E}-08$ & 4.2 & $0.5244 \mathrm{E}-08$ \\
1.8 & $0.9380 \mathrm{E}-08$ & 4.3 & $0.5125 \mathrm{E}-08$ \\
1.9 & $0.9204 \mathrm{E}-08$ & 4.4 & $0.5010 \mathrm{E}-08$ \\
2.0 & $0.9015 \mathrm{E}-08$ & 4.5 & $0.4899 \mathrm{E}-08$ \\
2.1 & $0.8818 \mathrm{E}-08$ & 4.6 & $0.4791 \mathrm{E}-08$ \\
2.2 & $0.8615 \mathrm{E}-08$ & 4.7 & $0.4687 \mathrm{E}-08$ \\
2.3 & $0.8410 \mathrm{E}-08$ & 4.8 & $0.4587 \mathrm{E}-08$ \\
2.4 & $0.8205 \mathrm{E}-08$ & 4.9 & $0.4490 \mathrm{E}-08$ \\
2.5 & $0.8002 \mathrm{E}-08$ & 5.0 & $0.4396 \mathrm{E}-08$ \\
\hline & & & \\
\hline
\end{tabular}

Table 17 e- $\mathrm{N}_{2}$ vibrational de-excitation rate coefficients, $\mathrm{cm}^{3} / \mathrm{s}$ $\left(v_{i}=2, J=50\right)$

\begin{tabular}{cccccc}
$\mathrm{T}, \mathrm{eV}$ & $\mathrm{v}_{\mathrm{f}}=1$ & $\mathrm{v}_{\mathrm{f}}=0$ & $\mathrm{~T}, \mathrm{eV}$ & $\mathrm{v}_{\mathrm{f}}=1$ & $\mathrm{v}_{\mathrm{f}}=0$ \\
\hline 0.1 & $0.1468 \mathrm{E}-11$ & $0.6325 \mathrm{E}-12$ & 2.6 & $0.6968 \mathrm{E}-08$ & $0.4190 \mathrm{E}-08$ \\
0.2 & $0.2056 \mathrm{E}-09$ & $0.1562 \mathrm{E}-09$ & 2.7 & $0.6803 \mathrm{E}-08$ & $0.4063 \mathrm{E}-08$ \\
0.3 & $0.1080 \mathrm{E}-08$ & $0.9254 \mathrm{E}-09$ & 2.8 & $0.6641 \mathrm{E}-08$ & $0.3941 \mathrm{E}-08$ \\
0.4 & $0.2390 \mathrm{E}-08$ & $0.2105 \mathrm{E}-08$ & 2.9 & $0.6482 \mathrm{E}-08$ & $0.3824 \mathrm{E}-08$ \\
0.5 & $0.3768 \mathrm{E}-08$ & $0.3280 \mathrm{E}-08$ & 3.0 & $0.6326 \mathrm{E}-08$ & $0.3712 \mathrm{E}-08$ \\
0.6 & $0.5014 \mathrm{E}-08$ & $0.4250 \mathrm{E}-08$ & 3.1 & $0.6175 \mathrm{E}-08$ & $0.3603 \mathrm{E}-08$ \\
0.7 & $0.6052 \mathrm{E}-08$ & $0.4971 \mathrm{E}-08$ & 3.2 & $0.6028 \mathrm{E}-08$ & $0.3500 \mathrm{E}-08$ \\
0.8 & $0.6872 \mathrm{E}-08$ & $0.5469 \mathrm{E}-08$ & 3.3 & $0.5884 \mathrm{E}-08$ & $0.3400 \mathrm{E}-08$ \\
0.9 & $0.7490 \mathrm{E}-08$ & $0.5785 \mathrm{E}-08$ & 3.4 & $0.5745 \mathrm{E}-08$ & $0.3305 \mathrm{E}-08$ \\
1.0 & $0.7937 \mathrm{E}-08$ & $0.5963 \mathrm{E}-08$ & 3.5 & $0.5610 \mathrm{E}-08$ & $0.3214 \mathrm{E}-08$ \\
1.1 & $0.8242 \mathrm{E}-08$ & $0.6038 \mathrm{E}-08$ & 3.6 & $0.5480 \mathrm{E}-08$ & $0.3126 \mathrm{E}-08$ \\
1.2 & $0.8434 \mathrm{E}-08$ & $0.6038 \mathrm{E}-08$ & 3.7 & $0.5353 \mathrm{E}-08$ & $0.3042 \mathrm{E}-08$ \\
1.3 & $0.8535 \mathrm{E}-08$ & $0.5985 \mathrm{E}-08$ & 3.8 & $0.5230 \mathrm{E}-08$ & $0.2961 \mathrm{E}-08$ \\
1.4 & $0.8565 \mathrm{E}-08$ & $0.5894 \mathrm{E}-08$ & 3.9 & $0.5111 \mathrm{E}-08$ & $0.2884 \mathrm{E}-08$ \\
1.5 & $0.8541 \mathrm{E}-08$ & $0.5778 \mathrm{E}-08$ & 4.0 & $0.4996 \mathrm{E}-08$ & $0.2809 \mathrm{E}-08$ \\
1.6 & $0.8475 \mathrm{E}-08$ & $0.5645 \mathrm{E}-08$ & 4.1 & $0.4885 \mathrm{E}-08$ & $0.2738 \mathrm{E}-08$ \\
1.7 & $0.8377 \mathrm{E}-08$ & $0.5501 \mathrm{E}-08$ & 4.2 & $0.4777 \mathrm{E}-08$ & $0.2669 \mathrm{E}-08$ \\
1.8 & $0.8256 \mathrm{E}-08$ & $0.5350 \mathrm{E}-08$ & 4.3 & $0.4672 \mathrm{E}-08$ & $0.2603 \mathrm{E}-08$ \\
1.9 & $0.8117 \mathrm{E}-08$ & $0.5198 \mathrm{E}-08$ & 4.4 & $0.4571 \mathrm{E}-08$ & $0.2539 \mathrm{E}-08$ \\
2.0 & $0.7966 \mathrm{E}-08$ & $0.5045 \mathrm{E}-08$ & 4.5 & $0.4473 \mathrm{E}-08$ & $0.2478 \mathrm{E}-08$ \\
2.1 & $0.7806 \mathrm{E}-08$ & $0.4893 \mathrm{E}-08$ & 4.6 & $0.4379 \mathrm{E}-08$ & $0.2419 \mathrm{E}-08$ \\
2.2 & $0.7641 \mathrm{E}-08$ & $0.4744 \mathrm{E}-08$ & 4.7 & $0.4287 \mathrm{E}-08$ & $0.2363 \mathrm{E}-08$ \\
2.3 & $0.7473 \mathrm{E}-08$ & $0.4599 \mathrm{E}-08$ & 4.8 & $0.4198 \mathrm{E}-08$ & $0.2308 \mathrm{E}-08$ \\
2.4 & $0.7304 \mathrm{E}-08$ & $0.4458 \mathrm{E}-08$ & 4.9 & $0.4112 \mathrm{E}-08$ & $0.2256 \mathrm{E}-08$ \\
2.5 & $0.7135 \mathrm{E}-08$ & $0.4322 \mathrm{E}-08$ & 5.0 & $0.4029 \mathrm{E}-08$ & $0.2205 \mathrm{E}-08$ \\
\hline
\end{tabular}


Table 18 e-N 2 vibrational de-excitation rate coefficients, $\mathrm{cm}^{3} / \mathrm{s}$ $\left(v_{i}=3, J=50\right)$

$\mathrm{T}, \mathrm{eV} \quad \mathrm{v}_{\mathrm{f}}=2 \quad 1 \quad 0$

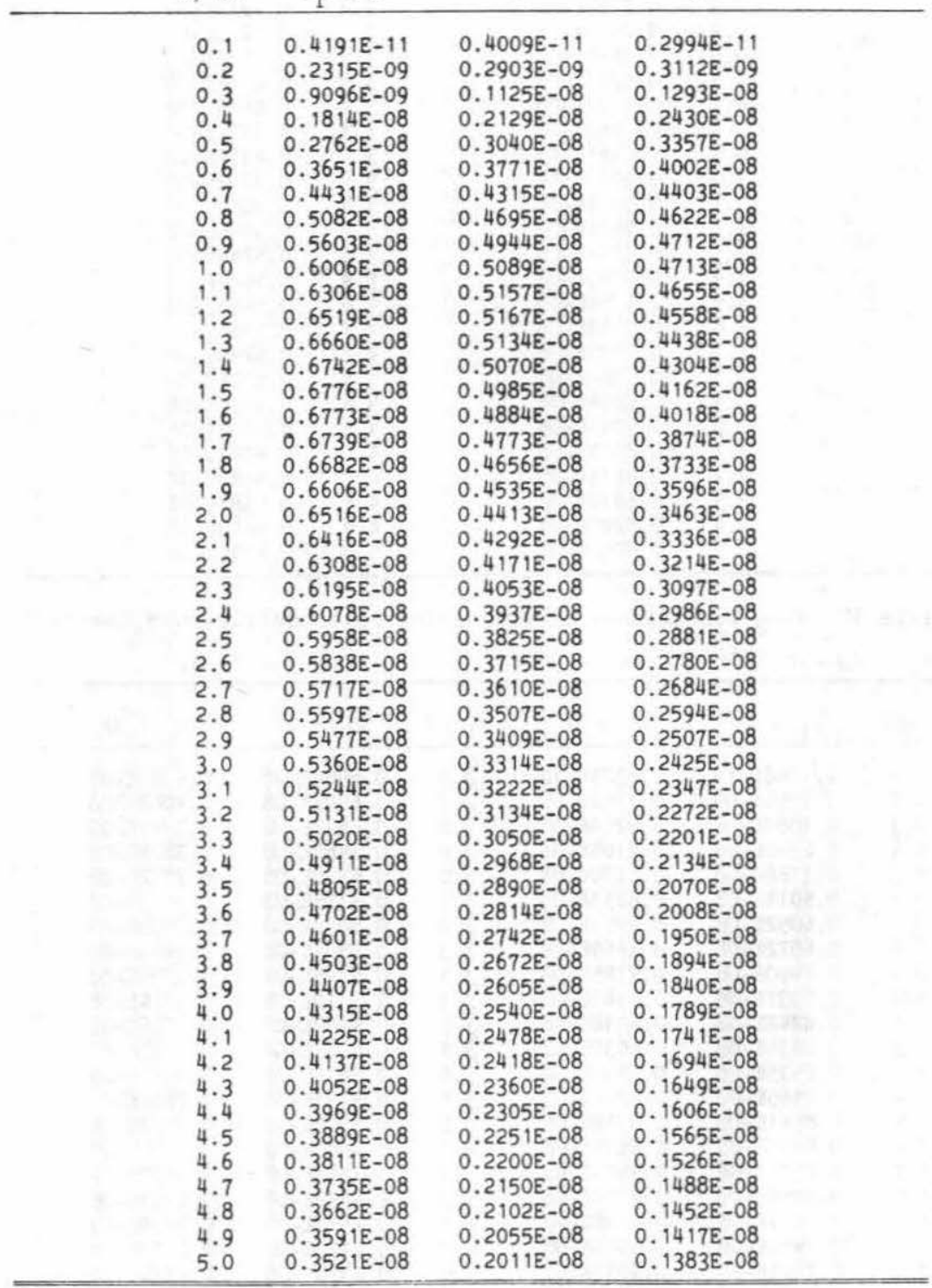


Table 19 e-N 2 vibrational de-excitation rate coefficients, $\mathrm{cm}^{3} / \mathrm{s}$ $\left(v_{i}=4, J=50\right)$

\begin{tabular}{|c|c|c|c|c|}
\hline $\mathrm{T}$, ev & $v_{f}=3$ & 2 & 1 & 0 \\
\hline 0.1 & $0.9359 \mathrm{E}-11$ & $0.9300 E-11$ & $0.1122 E-10$ & $0.1294 E-10$ \\
\hline 0.2 & $0.2960 \mathrm{E}-09$ & $0.2908 \mathrm{E}-09$ & $0.3523 \mathrm{E}-09$ & $0.5445 E-09$ \\
\hline 0.3 & $0.9583 E-09$ & $0.9236 E-09$ & $0.1070 E-08$ & $0.1655 E-08$ \\
\hline 0.4 & $0.1744 E-08$ & $0.1637 E-08$ & $0.1815 E-08$ & $0.2640 E-08$ \\
\hline 0.5 & 0.2534 E- 08 & $0.2294 E-08$ & $0.2428 E-08$ & $0.3294 E-08$ \\
\hline 0.6 & $0.3276 E-08$ & $0.2846 \mathrm{E}-08$ & $0.2876 E-08$ & $0.3663 E-08$ \\
\hline 0.7 & $0.3936 \mathrm{E}-08$ & $0.3281 E-08$ & $0.3176 E-08$ & $0.3830 \mathrm{E}-08$ \\
\hline 0.8 & $0.4499 E-08$ & $0.3608 \mathrm{E}-08$ & $0.3361 \mathrm{E}-08$ & $0.3868 \mathrm{E}-08$ \\
\hline 0.9 & $0.4961 \mathrm{E}-08$ & $0.3841 \mathrm{E}-08$ & $0.3457 \mathrm{E}-08$ & $0.3824 E-08$ \\
\hline 1.0 & $0.5329 \mathrm{E}-08$ & $0.3997 E-08$ & $0.3490 \mathrm{E}-08$ & $0.3731 \mathrm{E}-08$ \\
\hline 1.1 & $0.5612 E-08$ & $0.4092 E-08$ & $0.3478 E-08$ & $0.3610 E-08$ \\
\hline 1.2 & $0.5821 E-08$ & $0.4138 E-08$ & $0.3435 E-08$ & $0.3475 E-08$ \\
\hline 1.3 & $0.5968 E-08$ & $0.4147 \mathrm{E}-08$ & $0.3371 \varepsilon-08$ & $0.3334 E-08$ \\
\hline 1.4 & $0.6062 E-08$ & $0.4128 E-08$ & $0.3292 E-08$ & $0.3192 E-08$ \\
\hline 1.5 & $0.6114 E-08$ & $0.4087 \varepsilon-08$ & $0.3205 E-08$ & $0.3053 \mathrm{E}-08$ \\
\hline 1.6 & $0.6130 \mathrm{E}-08$ & $0.4031 \varepsilon-08$ & $0.3113 E-08$ & $0.2919 \mathrm{E}-08$ \\
\hline 1.7 & $0.6118 \mathrm{E}-08$ & $0.3963 E-08$ & $0.3018 \mathrm{E}-08$ & $0.2790 \mathrm{E}-08$ \\
\hline 1.8 & $0.6084 E-08$ & $0.3887 \varepsilon-08$ & $0.2923 E-08$ & $0.2668 \varepsilon-08$ \\
\hline 1.9 & $0.6031 \varepsilon-08$ & $0.3805 E-08$ & $0.2829 \mathrm{E}-08$ & $0.2552 E-08$ \\
\hline 2.0 & $0.5964 E-08$ & $0.3720 E-08$ & $0.2736 E-08$ & $0.2443 E-08$ \\
\hline 2.1 & $0.5886 \mathrm{E}-08$ & $0.3633 \mathrm{E}-08$ & $0.2646 E-08$ & $0.2340 E-08$ \\
\hline 2.2 & $0.5800 E-08$ & $0.3545 E-08$ & $0.2559 \mathrm{E}-08$ & $0.2243 E-08$ \\
\hline 2.3 & $0.5708 E-08$ & $0.3457 E-08$ & $0.2475 E-08$ & $0.2151 \mathrm{E}-08$ \\
\hline 2.4 & $0.5611 \varepsilon-08$ & $0.3370 \varepsilon-08$ & $0.2394 E-08$ & $0.2065 E-08$ \\
\hline 2.5 & $0.5511 E-08$ & $0.3284 E-08$ & $0.2317 E-08$ & $0.1984 E-08$ \\
\hline 2.6 & $0.5408 \mathrm{E}-08$ & $0.3200 E-08$ & $0.2242 E-0 E$ & $0.1908 \mathrm{E}-08$ \\
\hline 2.7 & $0.5305 \mathrm{E}-08$ & $0.3117 \mathrm{E}-08$ & $0.2171 \mathrm{E}-08$ & $0.1836 \mathrm{E}-08$ \\
\hline 2.8 & $0.5202 E-08$ & $0.3037 \mathrm{E}-08$ & $0.2103 E-08$ & $0.1768 E-08$ \\
\hline 2.9 & $0.5098 \mathrm{E}-08$ & $0.2959 \mathrm{E}-08$ & $0.2037 E-08$ & $0.1704 E-08$ \\
\hline 3.0 & $0.4996 \mathrm{E}-08$ & $0.2883 \varepsilon-08$ & $0.1975 \mathrm{E}-08$ & $0.1644 E-08$ \\
\hline 3.1 & $0.4895 \mathrm{E}-08$ & $0.2810 E-08$ & $0.1915 \mathrm{E}-08$ & $0.1587 \mathrm{E}-08$ \\
\hline 3.2 & $0.4795 E-08$ & $0.2738 E-08$ & $0.1858 \mathrm{E}-08$ & $0.1532 \mathrm{E}-08$ \\
\hline 3.3 & $0.4696 \mathrm{E}-08$ & $0.2670 E-08$ & $0.1804 E-08$ & $0.1481 E-08$ \\
\hline 3.4 & $0.4600 \mathrm{E}-08$ & $0.2603 E-08$ & $0.1751 \mathrm{E}-08$ & $0.1433 E-08$ \\
\hline 3.5 & $0.4505 E-08$ & $0.2539 E-08$ & $0.1702 E-08$ & $0.1387 \varepsilon-08$ \\
\hline 3.6 & $0.4413 E-08$ & $0.2477 E-08$ & $0.1654 \mathrm{E}-08$ & $0.1343 E-08$ \\
\hline 3.7 & $0.4323 E-08$ & $0.2417 E-08$ & $0.1608 \mathrm{E}-08$ & $0.1301 \mathrm{E}-08$ \\
\hline 3.8 & $0.4234 \mathrm{E}-08$ & $0.2359 \mathrm{E}-08$ & $0.1564 E-08$ & $0.1262 E-08$ \\
\hline 3.9 & $0.4148 E-08$ & $0.2303 E-08$ & $0.1522 E-08$ & $0.1224 E-08$ \\
\hline 4.0 & $0.4065 \mathrm{E}-08$ & $0.2249 E-08$ & $0.1482 E-08$ & $0.1189 E-08$ \\
\hline 4.1 & $0.3983 E-08$ & $0.2196 E-08$ & $0.1443 E-08$ & $0.1154 E-08$ \\
\hline 4.2 & $0.3903 E-08$ & $0.2146 E-08$ & $0.1406 E-08$ & $0.1122 \mathrm{E}-08$ \\
\hline 4.3 & $0.3826 \mathrm{E}-08$ & $0.2097 \mathrm{E}-08$ & $0.1371 E-08$ & $0.1091 \mathrm{E}-08$ \\
\hline 4.4 & $0.3751 E-08$ & $0.2050 E-08$ & $0.1337 E-08$ & $0.1061 E-08$ \\
\hline 4.5 & $0.3677 \mathrm{E}-08$ & $0.2005 E-08$ & $0.1304 \mathrm{E}-08$ & $0.1033 E-08$ \\
\hline 4.6 & $0.3606 \mathrm{E}-08$ & $0.1961 \mathrm{E}-08$ & $0.1272 E-08$ & $0.1005 E-08$ \\
\hline 4.7 & $0.3537 \mathrm{E}-08$ & $0.1919 E-08$ & $0.1242 E-08$ & $0.9793 \mathrm{E}-09$ \\
\hline 4.8 & $0.3469 \mathrm{E}-08$ & $0.1878 E-08$ & $0.1213 E-08$ & $0.9544 E-09$ \\
\hline 4.9 & $0.3404 E-08$ & $0.1838 E-08$ & $0.1185 E-08$ & $0.9304 E-09$ \\
\hline 5.0 & $0.3340 \mathrm{E}-08$ & $0.1800 \mathrm{E}-08$ & $0.1158 \mathrm{E}-08$ & $0.9075 E-09$ \\
\hline
\end{tabular}


Table 20 e- $\mathrm{N}_{2}$ vibrational de-excitation rate coefficients, $\mathrm{cm}^{3} / \mathrm{s}$ $\left(v_{1}=5, J=50\right)$

\begin{tabular}{|c|c|c|c|c|c|}
\hline $\mathrm{T}, \mathrm{eV}$ & $v_{f}=4$ & 3 & 2 & 1 & 0 \\
\hline 0.1 & $0.1724 E-10$ & $0.2124 \mathrm{E}-10$ & $0.2226 \mathrm{E}-10$ & $0.2447 \mathrm{E}-10$ & $0.4190 E-10$ \\
\hline 0.2 & $0.2983 \mathrm{E}-09$ & $0.3668 \mathrm{E}-09$ & $0.4325 \mathrm{E}-09$ & $0.3870 \mathrm{E}-09$ & $0.7799 \mathrm{E}-09$ \\
\hline 0.3 & $0.8107 E-09$ & $0.9488 \mathrm{E}-09$ & $0.1124 E-08$ & $0.9990 E-09$ & $0.1806 \mathrm{E}-08$ \\
\hline 0.4 & $0.1399 \varepsilon-08$ & $0.1540 \mathrm{E}-08$ & $0.1766 \mathrm{E}-08$ & $0.1570 \mathrm{E}-08$ & $0.2507 \mathrm{E}-08$ \\
\hline 0.5 & $0.2015 E-08$ & $0.2075 \mathrm{E}-08$ & $0.2274 E-08$ & $0.1998 \mathrm{E}-08$ & $0.2871 \mathrm{E}-08$ \\
\hline 0.6 & $0.2624 E-08$ & $0.2532 E-08$ & $0.2644 E-08$ & $0.2280 \mathrm{E}-08$ & $0.3011 \mathrm{E}-08$ \\
\hline 0.7 & $0.3192 E-08$ & $0.2904 E-08$ & $0.2897 E-08$ & $0.2446 \mathrm{E}-08$ & $0.3018 \mathrm{E}-08$ \\
\hline 0.8 & $0.3696 \mathrm{E}-08$ & $0.3193 E-08$ & $0.3056 \mathrm{E}-08$ & $0.2527 \mathrm{E}-08$ & $0.2950 \mathrm{E}-08$ \\
\hline 0.9 & $0.4127 \mathrm{E}-08$ & $0.3409 \mathrm{E}-08$ & $0.3144 E-08$ & $0.2549 E-08$ & $0.2844 E-08$ \\
\hline 1.0 & $0.4483 \mathrm{E}-08$ & $0.3561 \mathrm{E}-08$ & $0.3178 \mathrm{E}-08$ & $0.2532 E-08$ & $0.2718 \mathrm{E}-08$ \\
\hline 1.1 & $0.4768 \mathrm{E}-08$ & $0.3661 \mathrm{E}-08$ & $0.3175 \mathrm{E}-08$ & $0.2488 E-08$ & $0.2586 \mathrm{E}-08$ \\
\hline 1.2 & $0.4990 \mathrm{E}-08$ & $0.3719 E-08$ & $0.3144 E-08$ & $0.2427 E-08$ & $0.2454 E-08$ \\
\hline 1.3 & $0.5155 \mathrm{E}-08$ & $0.3744 \mathrm{E}-08$ & $0.3093 E-08$ & $0.2357 E-08$ & $0.2326 \mathrm{E}-08$ \\
\hline 1.4 & $0.5273 E-08$ & $0.3742 E-08$ & $0.3030 \mathrm{E}-08$ & $0.2280 E-08$ & $0.2204 E-08$ \\
\hline 1.5 & $0.5351 E-08$ & $0.3720 \mathrm{E}-08$ & $0.2957 \mathrm{E}-08$ & $0.2202 E-08$ & $0.2089 \mathrm{E}-08$ \\
\hline 1.6 & $0.5395 E-08$ & $0.3682 E-08$ & $0.2880 E-08$ & $0.2123 E-08$ & $0.1982 E-08$ \\
\hline 1.7 & $0.5411 \mathrm{E}-08$ & $0.3633 E-08$ & $0.2799 E-08$ & $0.2045 E-08$ & $0.1881 \mathrm{E}-08$ \\
\hline 1.8 & $0.5404 \mathrm{E}-08$ & $0.3574 E-08$ & $0.2718 \mathrm{E}-08$ & $0.1969 \mathrm{E}-08$ & $0.1787 \mathrm{E}-08$ \\
\hline 1.9 & 0.5379 & $0.3510 \mathrm{E}-08$ & $0.2636 \mathrm{E}-08$ & $0.1895 E-08$ & $0.1700 E-08$ \\
\hline 2.0 & $0.5339 \mathrm{E}-08$ & $0.3441 \mathrm{E}-08$ & $0.2556 \mathrm{E}-08$ & $0.1824 E-08$ & $0.1619 \mathrm{E}-08$ \\
\hline 2.1 & $0.5287 \mathrm{E}-08$ & $0.3369 \varepsilon-08$ & $0.2477 \mathrm{E}-08$ & $0.1756 \mathrm{E}-08$ & $0.1543 E-08$ \\
\hline 2.2 & $0.5226 \mathrm{E}-08$ & $0.3296 E-08$ & $0.2400 \mathrm{E}$ & $0.1692 E-08$ & $0.1473 E-08$ \\
\hline 2.3 & $0.5158 E-08$ & $0.3221 E-08$ & 0.2 & $0.1630 \mathrm{E}-08$ & $0.1408 \mathrm{E}-08$ \\
\hline 2.4 & $0.5083 E-08$ & $0.3147 E-08$ & 0.2 & $0.1571 \mathrm{E}-08$ & $0.1347 \mathrm{E}-08$ \\
\hline 2.5 & $0.5005 E-08$ & $0.3073 E-08$ & $0.2183 \mathrm{E}-08$ & $0.1515 E-08$ & $0.1290 \mathrm{E}-08$ \\
\hline 2.6 & $0.4923 E-08$ & $0.3000 \mathrm{E}-08$ & 0.2 & $0.1462 E-08$ & $0.1236 \mathrm{E}-08$ \\
\hline 2.7 & $0.4839 \mathrm{E}-08$ & $0.2928 \mathrm{E}-08$ & $0.2052 E-08$ & $0.1412 E-08$ & $0.1186 E-08$ \\
\hline 2.8 & $0.4754 \mathrm{E}-08$ & $0.2857 \mathrm{E}-08$ & 0.1990 & $0.1364 \mathrm{E}-08$ & $0.1139 E-08$ \\
\hline 2.9 & $0.4668 \varepsilon-08$ & $0.2788 \mathrm{E}-08$ & 0.1931 & $0.1318 \mathrm{E}-08$ & $0.1096 \mathrm{E}-08$ \\
\hline 3.0 & $0.4582 E-08$ & $0.2721 E-08$ & $0.1874 \mathrm{E}-08$ & $0.1275 E-08$ & $0.1054 \mathrm{E}-08$ \\
\hline 3.1 & $0.4497 \varepsilon-08$ & $0.2655 E-08$ & 0.181 & 0.1234 & $0.1016 \mathrm{E}-08$ \\
\hline 3.2 & $0.4412 E-08$ & $8-08$ & 0.1 & $0.1194 E-08$ & $0.9789 \mathrm{E}-09$ \\
\hline 3.3 & $0.4327 E-08$ & $0.2530 \mathrm{E}-08$ & $0.1717 \mathrm{E}-08$ & $0.1157 \varepsilon-08$ & $0.9445 E-09$ \\
\hline 3.4 & 0.42 & $E-08$ & 0.1669 & 0.112 & $0.9119 \mathrm{E}-09$ \\
\hline 3.5 & $0.4162 E-08$ & $0.2411 \mathrm{E}-08$ & $0.1623 \mathrm{E}-08$ & $0.1088 \mathrm{E}-08$ & $0.8811 \mathrm{E}-09$ \\
\hline 3.6 & $0.4082 E-08$ & $0.2355 E-08$ & 0.157 & $0.1055 E-08$ & $0.8520 \mathrm{E}-09$ \\
\hline 3.7 & $0.4003 E-08$ & $0.2300 \mathrm{E}-08$ & & 0.1 & $0.8244 E-09$ \\
\hline 3.8 & $0.3925 E-08$ & $0.2247 \mathrm{E}-08$ & $0.1496 \mathrm{E}-08$ & $0.9953 E-09$ & $0.7983 E-09$ \\
\hline 3.9 & $0.3850 \mathrm{E}-08$ & & & & \\
\hline 4.0 & $0.3776 \mathrm{E}-08$ & $0.2146 \mathrm{E}-08$ & 0.1 & $0.9404 \mathrm{E}-09$ & $0.7499 E-09$ \\
\hline 4.1 & $0.3703 E-08$ & $0.2099 \mathrm{E}-08$ & $0.1383 E-08$ & $0.9148 \mathrm{E}-09$ & $0.7275 E-09$ \\
\hline 4.2 & $0.3633 \mathrm{E}-08$ & $0.2052 E-08$ & 0.134 & $0.8902 E-09$ & $0.7061 \mathrm{E}-09$ \\
\hline 4.3 & $0.3564 \mathrm{E}-08$ & $0.2007 E-08$ & $0.1316 \mathrm{E}-$ & $0.8667 \mathrm{E}-09$ & $0.6858 \mathrm{E}-09$ \\
\hline 4.4 & $0.3496 \mathrm{E}-08$ & $0.1964 E-08$ & $0.1284 E-08$ & $0.8442 E-09$ & $0.6664 E-09$ \\
\hline 4.5 & $0.3431 \mathrm{E}-08$ & $E-08$ & $0.1253 \mathrm{E}-08$ & $0.8226 \mathrm{E}-09$ & $0.6479 E-09$ \\
\hline 4.6 & $0.3367 \mathrm{E}-08$ & $0.1881 E-08$ & $0.1223 E-08$ & $0.8019 E-09$ & $0.6302 E-09$ \\
\hline 4.7 & $0.3304 E-08$ & $0.1841 E-08$ & $0.1195 E-$ & $0.7820 \mathrm{E}-09$ & $0.6133 E-09$ \\
\hline 4.8 & $0.3244 E-08$ & $0.1803 \mathrm{E}-0$ & $0.1167 \mathrm{E}-$ & $0.7629 \mathrm{E}-09$ & $0.5971 \mathrm{E}-09$ \\
\hline 4.9 & $0.3184 E-08$ & $0.1766 \mathrm{E}-08$ & $0.1141 \mathrm{E}-$ & $0.7446 \mathrm{E}-09$ & $0.5817 E-09$ \\
\hline 5.0 & $0.3127 \mathrm{E}-08$ & $0.1730 \mathrm{E}-08$ & $0.1116 \mathrm{E}-08$ & $0.7269 \mathrm{E}-09$ & $0.5668 \mathrm{E}-09$ \\
\hline
\end{tabular}


Table 21 e-N 2 vibrational de-excitation rate coefficients, $\mathrm{cm}^{3} / \mathrm{s}$ $\left(v_{1}=6, J=50\right)$

\begin{tabular}{|c|c|c|c|c|c|}
\hline $\mathrm{T}, \mathrm{eV}$ & $v_{f}=5$ & 4 & 3 & 2 & 1 \\
\hline 0.1 & $0.2743 E-10$ & $0.3552 E-10$ & $0.4274 \mathrm{E}-10$ & $0.5370 E-10$ & $0.4456 \mathrm{E}-10$ \\
\hline 0.2 & $0.3071 \mathrm{E}-09$ & $0.3869 \mathrm{E}-09$ & $0.4295 \mathrm{E}-09$ & $0.5796 \mathrm{E}-09$ & $0.4859 \mathrm{E}-09$ \\
\hline 0.3 & $0.7337 \mathrm{E}-09$ & $0.8794 \mathrm{E}-09$ & $0.9461 \mathrm{E}-09$ & $0.1226 \mathrm{E}-08$ & $0.1088 \mathrm{E}-08$ \\
\hline 0.4 & $0.1216 \mathrm{E}-08$ & $0.1359 \mathrm{E}-08$ & $0.1418 \mathrm{E}-08$ & $0.1739 \mathrm{E}-08$ & $0.1556 \mathrm{E}-08$ \\
\hline 0.5 & $0.1736 \mathrm{E}-08$ & $0.1800 E-08$ & $0.1810 E-08$ & $0.2099 \mathrm{E}-08$ & $0.1852 E-08$ \\
\hline 0.6 & $0.2266 \mathrm{E}-08$ & $0.2190 \mathrm{E}-08$ & $0.2116 E-08$ & $0.2330 \mathrm{E}-08$ & $0.2011 \mathrm{E}-08$ \\
\hline 0.7 & $0.2776 \mathrm{E}-08$ & $0.2520 E-08$ & $0.2340 E-08$ & $0.2462 E-08$ & $0.2077 \mathrm{E}-08$ \\
\hline 0.8 & $0.3240 \mathrm{E}-08$ & $0.2787 \mathrm{E}-08$ & $0.2496 \mathrm{E}-08$ & $0.2524 E-08$ & $0.2082 E-08$ \\
\hline 0.9 & $0.3646 \mathrm{E}-08$ & $0.2995 \mathrm{E}-08$ & $0.2594 \mathrm{E}-08$ & $0.2535 \mathrm{E}-08$ & $0.2050 \mathrm{E}-08$ \\
\hline 1.0 & $0.3988 E-08$ & $0.3150 \mathrm{E}-08$ & $0.2649 \mathrm{E}-08$ & $0.2513 E-08$ & $0.1995 \mathrm{E}-08$ \\
\hline 1.1 & $0.4269 \mathrm{E}-08$ & $0.3260 \mathrm{E}-08$ & $0.2669 \mathrm{E}-08$ & $0.2467 \varepsilon-08$ & $0.1928 \mathrm{E}-08$ \\
\hline 1.2 & $0.4493 E-08$ & $0.3331 \varepsilon-08$ & $0.2664 \varepsilon-08$ & $0.2408 E-08$ & $0.1854 \mathrm{E}-08$ \\
\hline 1.3 & $0.4666 \mathrm{E}-08$ & $0.3372 E-08$ & $0.2640 E-08$ & $0.2339 \mathrm{E}-08$ & $0.1778 \varepsilon-08$ \\
\hline 1.4 & $0.4795 \mathrm{E}-08$ & $0.3387 \mathrm{E}-08$ & $0.2603 E-08$ & $0.2266 \mathrm{E}-08$ & $0.1703 E-08$ \\
\hline 1.5 & $0.4885 E-08$ & $0.3383 \mathrm{E}-08$ & $0.2556 \mathrm{E}-08$ & $0.2190 E-08$ & $0.1629 \mathrm{E}-08$ \\
\hline 1.6 & $0.4944 E-08$ & $0.3363 E-08$ & $0.2502 E-08$ & $0.2114 \mathrm{E}-08$ & $0.1557 \mathrm{E}-08$ \\
\hline 1.7 & $0.4975 E-08$ & $0.3331 \mathrm{E}-08$ & $0.2444 E-08$ & $0.2038 \mathrm{E}-08$ & $0.1489 \mathrm{E}-08$ \\
\hline 1.8 & $0.4984 \mathrm{E}-08$ & $0.3289 \mathrm{E}-08$ & $0.2383 E-08$ & $0.1965 E-08$ & $0.1424 \mathrm{E}-08$ \\
\hline 1.9 & $0.4975 E-08$ & $0.3240 \mathrm{E}-08$ & $0.2321 \mathrm{E}-08$ & $0.1894 \mathrm{E}-08$ & $0.1363 E-08$ \\
\hline 2.0 & $0.4950 \mathrm{E}-08$ & $0.3186 \mathrm{E}-08$ & $0.2258 \mathrm{E}-08$ & $0.1825 E-08$ & $0.1305 E-08$ \\
\hline 2.1 & $0.4914 E-08$ & $0.3129 \mathrm{E}-08$ & $0.2196 E-08$ & $0.1759 \mathrm{E}-08$ & $0.1250 \mathrm{E}-08$ \\
\hline 2.2 & $0.4867 \mathrm{E}-08$ & $0.3068 \mathrm{E}-08$ & $0.2134 \mathrm{E}-08$ & $0.1696 \mathrm{E}-08$ & $0.1199 \mathrm{E}-08$ \\
\hline 2.3 & $0.4813 E-08$ & $0.3006 \mathrm{E}-08$ & $0.2073 E-08$ & $0.1636 \mathrm{E}-08$ & $0.1150 \mathrm{E}-08$ \\
\hline 2.4 & $0.4752 \mathrm{E}-08$ & $0.2943 E-08$ & $0.2014 E-08$ & $0.1578 \varepsilon-08$ & $0.1105 E-08$ \\
\hline 2.5 & $0.4687 \mathrm{E}-08$ & $0.2880 \mathrm{E}-08$ & $0.1957 \varepsilon-08$ & $0.1524 E-08$ & $0.1062 E-08$ \\
\hline 2.6 & $0.4617 \mathrm{E}-08$ & $0.2817 \mathrm{E}-08$ & $0.1901 \mathrm{E}-08$ & $0.1471 E-08$ & $0.1021 \mathrm{E}-08$ \\
\hline 2.7 & $0.4545 E-08$ & $0.2754 E-08$ & $0.1848 E-08$ & $0.1422 \varepsilon-08$ & $0.9828 \mathrm{E}-09$ \\
\hline 2.8 & $0.4472 E-08$ & $0.2693 \mathrm{E}-08$ & $0.1796 \mathrm{E}-08$ & 0.137 & $0.9468 \mathrm{E}-09$ \\
\hline 2.9 & $0.4397 \mathrm{E}-08$ & $0.2632 E-08$ & $0.1745 E-08$ & $0.1330 \mathrm{E}-08$ & $0.9128 \mathrm{E}-09$ \\
\hline 3.0 & $0.4321 \mathrm{E}-08$ & $0.2572 E-08$ & $0.1697 \mathrm{E}-08$ & $0.1287 \varepsilon-08$ & $0.8806 \mathrm{E}-09$ \\
\hline 3.1 & $0.4245 E-08$ & $0.2514 \mathrm{E}-08$ & $0.1650 \mathrm{E}-$ & 0.124 & $0.8502 E-09$ \\
\hline 3.2 & $0.4169 \mathrm{E}-08$ & $0.2457 \mathrm{E}-08$ & $0.1606 \mathrm{E}-08$ & $0.1208 \mathrm{E}-08$ & $0.8215 \mathrm{E}-09$ \\
\hline 3.3 & $0.4094 E-08$ & $0.2401 \mathrm{E}-08$ & $0.1562 E-08$ & $0.1171 \mathrm{E}-08$ & $0.7942 \mathrm{E}-09$ \\
\hline 3.4 & $0.4019 \mathrm{E}-08$ & $0.2347 \mathrm{E}-08$ & $8-08$ & $0.1135 E-08$ & $0.7684 \mathrm{E}-09$ \\
\hline 3.5 & $0.3945 E-08$ & $0.2294 \mathrm{E}-08$ & $0.1481 \mathrm{E}-08$ & $0.1102 E-08$ & $0.7438 \mathrm{E}-09$ \\
\hline 3.6 & $0.3872 E-08$ & $0.2243 \mathrm{E}-08$ & $0.1442 E-08$ & $0.1070 \mathrm{E}-08$ & $0.7206 \mathrm{E}-09$ \\
\hline 3.7 & $0.3800 \mathrm{E}-08$ & $0.2193 \mathrm{E}-08$ & $0.1405 E-08$ & $0.1039 \mathrm{E}-08$ & $0.6984 \mathrm{E}-09$ \\
\hline 3.8 & $0.3730 \mathrm{E}-08$ & $0.2145 E-08$ & $0.1370 \mathrm{E}-08$ & 0.101 & $74 E-09$ \\
\hline 3.9 & $0.3660 \mathrm{E}-08$ & $0.2098 \mathrm{E}-08$ & $0.1336 \mathrm{E}-08$ & $0.9819 E-09$ & $0.6573 \mathrm{E}-09$ \\
\hline 4.0 & $0.3592 E-08$ & $0.2052 E-08$ & $0.1303 E-08$ & $0.9551 \mathrm{E}-09$ & $0.6382 \mathrm{E}-09$ \\
\hline 4.1 & $0.3526 \mathrm{E}-08$ & $0.2008 \mathrm{E}-08$ & $0.1271 \mathrm{E}-08$ & $0.9295 \mathrm{E}-09$ & $0.6200 \mathrm{E}-09$ \\
\hline 4.2 & $0.3461 \mathrm{E}-08$ & $0.1965 E-08$ & $0.1240 \mathrm{E}-08$ & $0.9049 \mathrm{E}-09$ & $0.6026 \mathrm{E}-09$ \\
\hline 4.3 & $0.3397 \mathrm{E}-08$ & $0.1924 E-08$ & $0.1211 \mathrm{E}-08$ & $0.8814 E-09$ & $0.5860 \mathrm{E}-09$ \\
\hline 4.4 & $0.3335 E-08$ & $0.1883 E-08$ & $0.1183 E-08$ & $0.8589 \mathrm{E}-09$ & $0.5701 \mathrm{E}-09$ \\
\hline 4.5 & $0.3274 E-08$ & $0.1844 \mathrm{E}-08$ & $0.1155 \mathrm{E}-08$ & $0.8373 E-09$ & $0.5549 \mathrm{E}-09$ \\
\hline 4.6 & $0.3215 E-08$ & $0.1807 \mathrm{E}-08$ & $0.1129 \mathrm{E}-08$ & $0.8165 E-09$ & $0.5404 \mathrm{E}-09$ \\
\hline 4.7 & $0.3157 \mathrm{E}-08$ & $0.1770 \mathrm{E}-08$ & $0.1103 E-08$ & $0.7965 \varepsilon-09$ & $0.5264 \mathrm{E}-09$ \\
\hline 4.8 & $0.3101 \mathrm{E}-08$ & $0.1734 \mathrm{E}-08$ & $0.1079 E-08$ & $0.7774 E-09$ & $0.5131 \varepsilon-09$ \\
\hline 4. & $0.3045 \mathrm{E}-0$ & 0.17 & 0.1 & $0.7589 \mathrm{E}-09$ & $0.5003 \mathrm{E}-09$ \\
\hline 5.0 & $0.2992 \mathrm{E}-0$ & $0.1666 \mathrm{E}-08$ & $0.1032 E-08$ & $0.7412 E-09$ & $0.4880 \mathrm{E}-09$ \\
\hline
\end{tabular}


Table 22 e- $\mathrm{N}_{2}$ vibrational excitation rate coefficients, $\mathrm{cm}^{3} / \mathrm{s}$ $\left(v_{i}=7, J=50\right)$

\begin{tabular}{|c|c|c|c|c|c|}
\hline $\mathrm{T}, \mathrm{eV}$ & $v_{f}=6$ & 5 & 4 & 3 & 2 \\
\hline 0.1 & $0.3673 E-10$ & $0.5155 E-10$ & $0.6970 \mathrm{E}-10$ & $0.7703 \mathrm{E}-10$ & $0.1010 \mathrm{E}-09$ \\
\hline 0.2 & $0.2897 \mathrm{E}-09$ & $0.3533 \mathrm{E}-09$ & $0.4797 \mathrm{E}-09$ & $0.5411 \mathrm{E}-09$ & $0.6092 \mathrm{E}-09$ \\
\hline 0.3 & $0.6392 \varepsilon-09$ & $0.7180 \mathrm{E}-09$ & $0.9285 \mathrm{E}-09$ & $0.1038 \mathrm{E}-08$ & $0.1112 E-08$ \\
\hline 0.4 & $0.1043 E-08$ & $0.1088 \mathrm{E}-08$ & $0.1320 \mathrm{E}-08$ & $0.1434 E-08$ & $0.1483 \mathrm{E}-08$ \\
\hline 0.5 & $0.1495 E-08$ & $0.1454 E-08$ & $0.1649 \mathrm{E}-08$ & $0.1727 \varepsilon-08$ & $0.1723 \mathrm{E}-08$ \\
\hline 0.6 & $0.1970 \mathrm{E}-08$ & $0.1798 \mathrm{E}-08$ & $0.1913 E-08$ & $0.1929 \mathrm{E}-08$ & $0.1859 \mathrm{E}-08$ \\
\hline 0.7 & $0.2437 E-08$ & $0.2104 E-08$ & $0.2114 \mathrm{E}-08$ & $0.2058 \mathrm{E}-08$ & $0.1921 \mathrm{E}-08$ \\
\hline 0.8 & $0.2871 \mathrm{E}-08$ & $0.2363 E-08$ & $0.2258 \mathrm{E}-08$ & $0.2129 \mathrm{E}-08$ & $0.1933 \mathrm{E}-08$ \\
\hline 0.9 & $0.3258 \mathrm{E}-08$ & $0.2573 E-08$ & $0.2356 \mathrm{E}-08$ & $0.2159 E-08$ & $0.1911 \mathrm{E}-08$ \\
\hline 1.0 & $0.3590 \mathrm{E}-08$ & $0.2738 \mathrm{E}-08$ & $0.2414 \mathrm{E}-08$ & $0.2157 E-08$ & $0.1870 \mathrm{E}-08$ \\
\hline 1.1 & $0.3867 E-08$ & $0.2862 E-08$ & $0.2443 \mathrm{E}-08$ & $0.2135 E-08$ & $0.1815 E-08$ \\
\hline 1.2 & $0.4092 \mathrm{E}-08$ & $0.2950 \mathrm{E}-08$ & $0.2448 \mathrm{E}-08$ & $0.2097 \mathrm{E}-08$ & $0.1754 \varepsilon-08$ \\
\hline 1.3 & $0.4270 E-08$ & $0.3009 \mathrm{E}-08$ & $0.2435 E-08$ & $0.2050 \mathrm{E}-08$ & $0.1689 \mathrm{E}-08$ \\
\hline 1.4 & $0.4407 \varepsilon-08$ & $0.3043 \mathrm{E}-08$ & $0.2409 \mathrm{E}-08$ & $0.1996 \mathrm{E}-08$ & $0.1624 E-08$ \\
\hline 1.5 & $0.4507 \mathrm{E}-08$ & $0.3057 \mathrm{E}-08$ & $0.2373 E-08$ & $0.1939 \mathrm{E}-08$ & $0.1559 \mathrm{E}-08$ \\
\hline 1.6 & $0.4576 \mathrm{E}-08$ & $0.3055 \mathrm{E}-08$ & $0.2330 \mathrm{E}-08$ & $0.1880 \mathrm{E}-08$ & $0.1495 \mathrm{E}-08$ \\
\hline 1.7 & $0.4619 E-08$ & $0.3040 \mathrm{E}-08$ & $0.2282 E-08$ & $0.1820 \mathrm{E}-08$ & $0.1434 \mathrm{E}-08$ \\
\hline 1.8 & $0.4640 \mathrm{E}-08$ & $0.3014 \mathrm{E}-08$ & $0.2231 \mathrm{E}-08$ & $0.1761 \mathrm{E}-08$ & $0.1376 \mathrm{E}-08$ \\
\hline 1.9 & $0.4643 E-08$ & $0.2981 \mathrm{E}-08$ & $0.2178 E-08$ & $0.1703 E-08$ & $0.1320 \mathrm{E}-08$ \\
\hline 2.0 & $0.4631 \mathrm{E}-08$ & $0.2942 \mathrm{E}-08$ & $0.2124 \mathrm{E}-08$ & $0.1646 \mathrm{E}-08$ & $0.1267 \mathrm{E}-08$ \\
\hline 2.1 & $0.4606 \mathrm{E}-08$ & $0.2897 \mathrm{E}-08$ & $0.2070 \mathrm{E}-08$ & $0.1591 E-08$ & $0.1216 \mathrm{E}-08$ \\
\hline 2.2 & $0.4571 \mathrm{E}-08$ & $0.2850 \mathrm{E}-08$ & $0.2016 \mathrm{E}-08$ & $0.1538 \mathrm{E}-08$ & $0.1169 \mathrm{E}-08$ \\
\hline 2.3 & $0.4528 \mathrm{E}-08$ & $0.2800 \mathrm{E}-08$ & $0.1962 E-08$ & $0.1487 \mathrm{E}-08$ & $0.1123 \mathrm{E}-08$ \\
\hline 2.4 & $0.4478 E-08$ & $0.2748 E-08$ & $0.1909 \mathrm{E}-08$ & $0.1438 E-08$ & $0.1081 E-08$ \\
\hline 2.5 & $0.4423 E-08$ & $0.2695 \mathrm{E}-08$ & $0.1858 \mathrm{E}-08$ & $0.1391 \mathrm{E}-08$ & $0.1040 \mathrm{E}-08$ \\
\hline 2.6 & $0.4363 E-08$ & $0.2641 \mathrm{E}-08$ & $0.1808 \mathrm{E}-08$ & $0.1346 \mathrm{E}-08$ & $0.1002 \mathrm{E}-08$ \\
\hline 2.7 & $0.4301 \mathrm{E}-08$ & $0.2588 \mathrm{E}-08$ & $0.1759 \mathrm{E}-08$ & $0.1303 E-08$ & $0.9662 \mathrm{E}-09$ \\
\hline 2.8 & $0.4236 \mathrm{E}-08$ & $0.2534 E-08$ & 0.1712 & $0.1262 E-08$ & $0.9320 \mathrm{E}-09$ \\
\hline 2.9 & $0.4170 \varepsilon-08$ & $0.2481 E-08$ & $0.1666 \mathrm{E}-08$ & $0.1223 E-08$ & $0.8997 \mathrm{E}-09$ \\
\hline 3.0 & $0.4102 E-08$ & $0.2429 \mathrm{E}-08$ & $0.1622 E-08$ & $0.1185 \mathrm{E}-08$ & $0.8691 \mathrm{E}-09$ \\
\hline 3.1 & $0.4034 E-08$ & $0.2377 \mathrm{E}-08$ & $0.1579 \mathrm{E}-08$ & $0.1149 \mathrm{E}-08$ & $0.8400 \mathrm{E}-09$ \\
\hline 3.2 & $0.3966 \mathrm{E}-08$ & $0.2327 \mathrm{E}-08$ & $0.1538 \mathrm{E}-08$ & $0.1115 \mathrm{E}-08$ & $0.8125 \varepsilon-09$ \\
\hline 3.3 & $0.3898 \mathrm{E}-08$ & $0.2277 \mathrm{E}-08$ & $0.1498 \mathrm{E}-08$ & $0.1082 E-08$ & $0.7864 \mathrm{E}-09$ \\
\hline 3.4 & $0.3830 E-08$ & $0.2228 \mathrm{E}-08$ & $0.1459 \mathrm{E}-08$ & $0.1051 \mathrm{E}-08$ & $0.7615 \mathrm{E}-09$ \\
\hline 3.5 & $0.3762 E-08$ & $0.2181 \mathrm{E}-08$ & $0.1422 E-08$ & $0.1021 E-08$ & $0.7379 \mathrm{E}-09$ \\
\hline 3.6 & $0.3695 \mathrm{E}-08$ & $0.2135 \mathrm{E}-08$ & $0.1387 \mathrm{E}-08$ & $0.9925 \mathrm{E}-09$ & $0.7154 \mathrm{E}-09$ \\
\hline 3.7 & $0.36298-08$ & $0.2089 \mathrm{E}-08$ & 0.13 & 0.96 & $0.6940 \mathrm{E}-09$ \\
\hline 3.8 & $0.3564 \mathrm{E}-08$ & $0.2045 E-08$ & $0.1319 \mathrm{E}-08$ & $0.9388 \mathrm{E}-09$ & $0.6736 \mathrm{E}-09$ \\
\hline 3.9 & & 0.2 & & & $0.6542 E-09$ \\
\hline 4.0 & $0.3438 E-08$ & $0.1961 \mathrm{E}-08$ & $0.1256 \mathrm{E}-08$ & $0.8895 E-09$ & $0.6356 \mathrm{E}-09$ \\
\hline 4.1 & $0.3376 E-08$ & $0.1920 \mathrm{E}-08$ & $0.1226 \mathrm{E}-08$ & $0.8663 E-09$ & $0.6179 \mathrm{E}-09$ \\
\hline 4.2 & $0.3315 E-08$ & $0.1881 \mathrm{E}-08$ & $0.1198 \mathrm{E}-08$ & $0.8442 E-09$ & $0.6010 \mathrm{E}-09$ \\
\hline 4.3 & $0.3256 E-08$ & $0.1843 E-08$ & $0.1170 \mathrm{E}-08$ & $0.8228 \mathrm{E}-09$ & $0.5848 \mathrm{E}-09$ \\
\hline 4.4 & $0.3198 \mathrm{E}-08$ & $0.1806 \mathrm{E}-08$ & $0.1143 E-08$ & $0.8024 E-09$ & $0.5693 E-09$ \\
\hline 4.5 & $0.3142 E-08$ & $0.1770 \mathrm{E}-08$ & $0.1117 \mathrm{E}-08$ & $0.7827 \mathrm{E}-09$ & $0.5544 \mathrm{E}-09$ \\
\hline 4.6 & $0.3086 \mathrm{E}-08$ & $0.1734 \varepsilon-08$ & $0.1093 \mathrm{E}-08$ & $0.7638 \mathrm{E}-09$ & $0.5402 \varepsilon-09$ \\
\hline 4.7 & $0.3032 \varepsilon-08$ & $0.1700 \varepsilon-08$ & $0.1068 \mathrm{E}-08$ & $0.7456 \mathrm{E}-09$ & $0.5266 \mathrm{E}-09$ \\
\hline 4.8 & $0.2979 E-08$ & $0.1667 \varepsilon-08$ & $0.1045 E-08$ & $0.7282 E-09$ & $0.5135 \mathrm{E}-09$ \\
\hline 4.9 & $0.2927 \mathrm{E}-0$ & $0.1635 \mathrm{E}-\mathrm{C}$ & $0.1023 E-08$ & $0.7113 E-09$ & $0.5009 \mathrm{E}-09$ \\
\hline 5.0 & $0.2877 \mathrm{E}-08$ & $0.1604 \mathrm{E}-08$ & $0.1001 \mathrm{E}-08$ & $0.6951 \mathrm{E}-09$ & $0.4889 \mathrm{E}-09$ \\
\hline
\end{tabular}


Table 23 e- $\mathrm{N}_{2}$ vibrational de-excitation rate coefficients, $\mathrm{cm}^{3} / \mathrm{s}$ $\left(v_{i}=8, J=50\right)$

\begin{tabular}{|c|c|c|c|c|c|}
\hline $\mathrm{T}$, & $v_{f}=7$ & 6 & 5 & 4 & 3 \\
\hline 0.1 & $0.4295 E-10$ & $0.6626 \mathrm{E}-10$ & $0.8972 \varepsilon-10$ & $0.1121 \mathrm{E}-09$ & $0.1411 \mathrm{E}-09$ \\
\hline 0.2 & $0.2450 \mathrm{E}-09$ & $0.3282 E-09$ & $0.4508 \mathrm{E}-09$ & $0.5063 \mathrm{E}-09$ & $0.6656 \mathrm{E}-09$ \\
\hline 0.3 & $0.5153 \mathrm{E}-09$ & $0.6186 \mathrm{E}-09$ & $0.8066 \mathrm{E}-09$ & $0.8813 E-09$ & $0.1096 \mathrm{E}-08$ \\
\hline 0.4 & $0.8523 \mathrm{E}-09$ & $0.9277 \varepsilon-09$ & $0.1124 \mathrm{E}-08$ & $0.1196 \mathrm{E}-08$ & $0.1398 \mathrm{E}-08$ \\
\hline 0.5 & $0.1251 \mathrm{E}-08$ & $0.1249 \mathrm{E}-08$ & $0.1405 \mathrm{E}-08$ & $0.1447 E-08$ & $0.1597 \varepsilon-08$ \\
\hline 0.6 & $0.1685 E-08$ & $0.1563 \mathrm{E}-08$ & $0.1641 \varepsilon-08$ & $0.1633 E-08$ & $0.1715 \mathrm{E}-08$ \\
\hline 0.7 & $0.2121 \mathrm{E}-08$ & $0.1851 \mathrm{E}-08$ & $0.1830 \mathrm{E}-08$ & $0.1761 \varepsilon-08$ & $0.1773 E-08$ \\
\hline 0.8 & $0.2534 \mathrm{E}-08$ & $0.2102 E-08$ & $0.1973 E-08$ & $0.1842 E-08$ & $0.1789 \mathrm{E}-08$ \\
\hline 0.9 & $0.2907 \mathrm{E}-08$ & $0.2311 \mathrm{E}-08$ & $0.2076 \mathrm{E}-08$ & $0.1885 E-08$ & $0.1776 E-08$ \\
\hline 1.0 & $0.3232 E-08$ & $0.2479 \mathrm{E}-08$ & $0.2144 E-08$ & $0.1899 \mathrm{E}-08$ & $0.1744 \mathrm{E}-08$ \\
\hline 1.1 & $0.3507 E-08$ & $0.2610 \mathrm{E}-08$ & $0.2184 E-08$ & $0.1893 E-08$ & $0.1700 \mathrm{E}-08$ \\
\hline 1.2 & $0.3735 E-08$ & $0.2708 \mathrm{E}-08$ & $0.2202 E-08$ & $0.1872 E-08$ & $0.1649 \mathrm{E}-08$ \\
\hline 1.3 & $0.3918 \mathrm{E}-08$ & $0.2777 \mathrm{E}-08$ & $0.2203 E-08$ & $0.1841 \mathrm{E}-08$ & $0.1594 \mathrm{E}-08$ \\
\hline 1.4 & $0.4062 \varepsilon-08$ & $0.2822 E-08$ & $0.2190 \mathrm{E}-08$ & $0.1802 E-08$ & $0.1537 \varepsilon-08$ \\
\hline 1.5 & $0.4170 E-08$ & 0.2847 E- 08 & $0.2167 \mathrm{E}-08$ & $0.1758 \mathrm{E}-08$ & $0.1480 E-08$ \\
\hline 1.6 & $0.4249 \mathrm{E}-08$ & $0.2856 \mathrm{E}-08$ & $0.2136 \mathrm{E}-08$ & $0.1712 E-08$ & $0.1424 E-08$ \\
\hline 1.7 & $0.4303 E-08$ & $0.2852 \mathrm{E}-08$ & $0.2100 \mathrm{E}-08$ & $0.1663 E-08$ & $0.1369 \mathrm{E}-08$ \\
\hline 1.8 & $0.4334 \mathrm{E}-08$ & 0.2837 E- 08 & $0.2060 \mathrm{E}-08$ & $0.1615 E-08$ & $0.1316 \mathrm{E}-08$ \\
\hline 1.9 & $0.4348 \mathrm{E}-08$ & $0.2814 \mathrm{E}-08$ & 0.2017 E- 08 & $0.1566 \mathrm{E}-08$ & $0.1266 \mathrm{E}-08$ \\
\hline 2.0 & $0.4346 \varepsilon-08$ & $0.2784 E-08$ & $0.1972 E-08$ & $0.1518 \mathrm{E}-08$ & $0.1217 \mathrm{E}-08$ \\
\hline 2.1 & $0.4331 E-08$ & $0.2748 \mathrm{E}-08$ & $0.1927 \mathrm{E}-08$ & $0.1471 \mathrm{E}-08$ & $0.1171 \mathrm{E}-08$ \\
\hline 2.2 & $0.4306 E-08$ & $0.2709 \mathrm{E}-08$ & $0.1881 \mathrm{E}-08$ & $0.1426 \mathrm{E}-08$ & $0.1127 \varepsilon-08$ \\
\hline 2.3 & $0.4273 \mathrm{E}-08$ & $0.2667 \mathrm{E}-08$ & $0.1835 \mathrm{E}-08$ & $0.1381 \mathrm{E}-08$ & $0.1085 E-08$ \\
\hline 2.4 & $0.4232 E-08$ & $0.2622 \mathrm{E}-08$ & $0.1789 \mathrm{E}-08$ & $0.1338 E-08$ & $0.1046 E-08$ \\
\hline 2.5 & $0.4186 \mathrm{E}-08$ & $0.2576 \mathrm{E}-08$ & $0.1744 \varepsilon-08$ & $0.1297 \mathrm{E}-08$ & $0.1008 \mathrm{E}-08$ \\
\hline 2.6 & $0.4136 \mathrm{E}-08$ & $0.2529 \mathrm{E}-08$ & $0.1700 \mathrm{E}-08$ & $0.1257 \mathrm{E}-08$ & $0.9726 \mathrm{E}-09$ \\
\hline 2.7 & $0.4081 \mathrm{E}-08$ & $0.2481 \mathrm{E}-08$ & $0.1657 \mathrm{E}-08$ & $0.1219 \mathrm{E}-08$ & $0.9387 \varepsilon-09$ \\
\hline 2.8 & $0.4025 \mathrm{E}-08$ & $0.2434 E-08$ & $0.1615 \mathrm{E}-08$ & $0.1183 E-08$ & $0.9066 \mathrm{E}-09$ \\
\hline 2.9 & $0.3966 \mathrm{E}-08$ & $0.2386 E-08$ & $0.1574 E-08$ & $0.1147 \mathrm{E}-08$ & $0.8762 E-09$ \\
\hline 3.0 & $0.3906 \mathrm{E}-08$ & $0.2338 \mathrm{E}-08$ & $0.1534 E-08$ & $0.1114 \mathrm{E}-08$ & $0.8472 E-09$ \\
\hline 3.1 & $0.3844 E-08$ & $0.2291 \mathrm{E}-08$ & $0.1495 \mathrm{E}-08$ & $0.1081 \mathrm{E}-08$ & $0.8197 \mathrm{E}-09$ \\
\hline 3.2 & $0.3783 E-08$ & $0.2245 \mathrm{E}-08$ & $0.1458 \mathrm{E}-08$ & $0.1050 E-08$ & $0.7936 \mathrm{E}-09$ \\
\hline 3.3 & $0.3720 \mathrm{E}-08$ & $0.2199 \mathrm{E}-08$ & $0.1422 E-08$ & $0.1021 \mathrm{E}-08$ & $0.7687 \mathrm{E}-09$ \\
\hline 3.4 & $0.3658 \varepsilon-08$ & $0.2154 \mathrm{E}-08$ & $0.1387 \mathrm{E}-08$ & $0.9920 E-09$ & $0.7451 \varepsilon-09$ \\
\hline 3.5 & $0.3597 \mathrm{E}-08$ & $0.2110 \mathrm{E}-08$ & $0.1353 E-08$ & $0.9647 \mathrm{E}-09$ & $0.7225 \mathrm{E}-09$ \\
\hline 3.6 & $0.3535 \mathrm{E}-08$ & $0.2067 \varepsilon-08$ & $0.1320 \mathrm{E}-08$ & $0.9385 \mathrm{E}-09$ & $0.7010 \mathrm{E}-09$ \\
\hline 3.7 & $0.3474 \mathrm{E}-08$ & $0.2025 E-08$ & $0.1288 \mathrm{E}-08$ & $0.9134 E-09$ & $0.6806 \mathrm{E}-09$ \\
\hline 3.8 & $0.3414 E-08$ & $0.1984 E-08$ & 0.125 & & $0.6610 \mathrm{E}-09$ \\
\hline 3.9 & $0.3355 \varepsilon-08$ & $0.1944 E-08$ & $0.1228 \mathrm{E}-08$ & $0.8662 E-09$ & $0.6424 E-09$ \\
\hline 4.0 & $0.3297 \mathrm{E}-08$ & $0.1905 \mathrm{E}-08$ & $0.1200 \mathrm{E}-08$ & $0.8439 \mathrm{E}-09$ & $0.6245 E-09$ \\
\hline 4.1 & $0.3239 E-08$ & $0.1867 E-08$ & $0.1172 E-08$ & $0.8226 \mathrm{E}-09$ & $0.6075 E-09$ \\
\hline 4.2 & $0.3183 E-08$ & $0.1830 E-08$ & $0.1146 \mathrm{E}-08$ & $0.8021 E-09$ & $0.5912 E-09$ \\
\hline 4.3 & $0.3128 \mathrm{E}-08$ & $0.1794 \mathrm{E}-08$ & $0.1120 \varepsilon-08$ & $0.7824 E-09$ & $0.5756 \mathrm{E}-09$ \\
\hline 4.4 & $0.3074 \mathrm{E}-08$ & $0.1759 \mathrm{E}-08$ & $0.1095 \mathrm{E}-08$ & $0.7634 \mathrm{E}-09$ & $0.5606 \mathrm{E}-09$ \\
\hline 4.5 & 0.3020 E- 08 & $0.1724 \mathrm{E}-08$ & $0.1071 E-08$ & $0.7452 E-09$ & $0.5462 E-09$ \\
\hline 4.6 & $0.2968 \mathrm{E}-08$ & $0.1691 \mathrm{E}-08$ & $0.1048 \mathrm{E}-08$ & $0.7276 \mathrm{E}-09$ & $0.5325 E-09$ \\
\hline 4.7 & $0.2917 \mathrm{E}-08$ & $0.1659 \mathrm{E}-08$ & $0.1025 E-08$ & 0.7107 E-09 & $0.5193 E-09$ \\
\hline 4.8 & $0.2868 \mathrm{E}-08$ & $0.1627 \varepsilon-08$ & $0.1004 \mathrm{E}-08$ & $0.6944 \mathrm{E}-09$ & $0.5066 \mathrm{E}-09$ \\
\hline 4.9 & $0.2819 E-08$ & $0.1597 E-08$ & $0.9826 \mathrm{E}-09$ & $0.6787 \mathrm{E}-09$ & $0.4944 E-09$ \\
\hline 5.0 & $0.2771 E-08$ & $0.1567 \mathrm{E}-$ & $0.9623 \mathrm{E}-09$ & $0.6635 \mathrm{E}-09$ & $0.4827 E-09$ \\
\hline
\end{tabular}


Table 24 e- $\mathrm{N}_{2}$ vibrational excitation rate coefficients, $\mathrm{cm}^{3} / \mathrm{s}$ $\left(v_{i}=9, J=50\right)$

\begin{tabular}{|c|c|c|c|c|c|}
\hline $\mathrm{T}, \mathrm{eV}$ & $v_{f}=8$ & 7 & 6 & 5 & 4 \\
\hline 0.1 & $0.4533 E-10$ & $0.7321 \mathrm{E}-10$ & $0.1020 \mathrm{E}-09$ & $0.1433 \mathrm{E}-09$ & $0.1559 \mathrm{E}-09$ \\
\hline 0.2 & $0.2015 E-09$ & $0.2905 \mathrm{E}-09$ & $0.3755 \mathrm{E}-09$ & $0.4968 \mathrm{E}-09$ & $0.5584 \mathrm{E}-09$ \\
\hline 0.3 & $0.4194 E-09$ & $0.5293 \mathrm{E}-09$ & $0.6426 \mathrm{E}-09$ & $0.8037 \varepsilon-09$ & $0.8833 \mathrm{E}-09$ \\
\hline 0.4 & $0.7137 \mathrm{E}-09$ & $0.7975 E-09$ & $0.9054 \mathrm{E}-09$ & $0.1069 \mathrm{E}-08$ & $0.1134 E-08$ \\
\hline 0.5 & $0.1076 \mathrm{E}-08$ & $0.1088 \mathrm{E}-08$ & $0.1155 \mathrm{E}-08$ & $0.1290 \mathrm{E}-08$ & $0.1317 \mathrm{E}-08$ \\
\hline 0.6 & $0.1479 \mathrm{E}-08$ & $0.1380 \mathrm{E}-08$ & $0.1376 E-08$ & $0.1461 \mathrm{E}-08$ & $0.1438 \mathrm{E}-08$ \\
\hline 0.7 & $0.1890 \mathrm{E}-08$ & $0.1654 E-08$ & $0.1562 E-08$ & $0.1584 \mathrm{E}-08$ & $0.1510 E-08$ \\
\hline 0.8 & $0.2284 E-08$ & $0.1898 \mathrm{E}-08$ & $0.1708 \mathrm{E}-08$ & $0.1666 \mathrm{E}-08$ & $0.1544 E-08$ \\
\hline 0.9 & $0.2644 \mathrm{E}-08$ & $0.2105 E-08$ & $0.1819 E-08$ & $0.1715 \mathrm{E}-08$ & $0.1550 \mathrm{E}-08$ \\
\hline 1.0 & $0.2961 \mathrm{E}-08$ & $0.2276 \mathrm{E}-08$ & $0.1898 \mathrm{E}-08$ & $0.1738 \mathrm{E}-08$ & $0.1537 \mathrm{E}-08$ \\
\hline 1.1 & $0.3233 E-08$ & $0.2411 \mathrm{E}-08$ & $0.1950 \mathrm{E}-08$ & $0.1741 \mathrm{E}-08$ & $0.1511 \mathrm{E}-08$ \\
\hline 1.2 & $0.3459 \mathrm{E}-08$ & $0.2515 E-08$ & $0.1981 \mathrm{E}-08$ & $0.1729 \mathrm{E}-08$ & $0.1476 E-08$ \\
\hline 1.3 & $0.3644 \mathrm{E}-08$ & $0.2592 E-08$ & $0.1994 \mathrm{E}-08$ & $0.1707 \mathrm{E}-08$ & $0.1436 \mathrm{E}-08$ \\
\hline 1.4 & $0.3792 E-08$ & $0.2646 \mathrm{E}-08$ & $0.1994 E-08$ & $0.1677 \varepsilon-08$ & $0.1392 \mathrm{E}-08$ \\
\hline 1.5 & $0.3906 \mathrm{E}-08$ & $0.2680 \mathrm{E}-08$ & $0.1982 E-08$ & $0.1641 E-08$ & $0.1347 \mathrm{E}-08$ \\
\hline 1.6 & $0.3991 \mathrm{E}-08$ & $0.2698 \mathrm{E}-08$ & $0.1963 E-08$ & $0.1603 E-08$ & $0.1302 E-08$ \\
\hline 1.7 & $0.4051 \mathrm{E}-08$ & $0.2702 E-08$ & $0.1937 \varepsilon-08$ & $0.1562 \mathrm{E}-08$ & $0.1257 E-08$ \\
\hline 1.8 & $0.4090 \mathrm{E}-08$ & $0.2695 E-08$ & $0.1907 E-08$ & $0.1520 \mathrm{E}-08$ & $0.1212 E-08$ \\
\hline 1.9 & $0.4111 \mathrm{E}-08$ & $0.2680 \mathrm{E}-08$ & $0.1873 E-08$ & $0.1477 \mathrm{E}-08$ & $0.1169 \mathrm{E}-08$ \\
\hline 2.0 & $0.4116 \mathrm{E}-08$ & $0.2657 \mathrm{E}-08$ & $0.1837 \mathrm{E}-08$ & $0.1435 E-08$ & $0.1128 \mathrm{E}-08$ \\
\hline 2.1 & $0.4109 \mathrm{E}-08$ & $0.2629 \mathrm{E}-08$ & $0.1799 \mathrm{E}-08$ & $0.1393 E-08$ & $0.1088 \mathrm{E}-08$ \\
\hline 2.2 & $0.4092 \mathrm{E}-08$ & $0.2597 \mathrm{E}-08$ & $0.1760 \mathrm{E}-08$ & $0.1353 \mathrm{E}-08$ & $0.1050 E-08$ \\
\hline 2.3 & $0.4065 \mathrm{E}-08$ & $0.2561 E-08$ & $0.1721 E-08$ & $0.1313 E-08$ & $0.1013 \mathrm{E}-08$ \\
\hline 2.4 & $0.4032 E-08$ & $0.2522 E-08$ & $0.1682 E-08$ & $0.1274 E-08$ & $0.9780 \mathrm{E}-09$ \\
\hline 2.5 & $0.3993 \mathrm{E}-08$ & $0.2482 E-08$ & $0.1643 E-08$ & $0.1236 \mathrm{E}-08$ & $0.9447 \mathrm{E}-09$ \\
\hline 2.6 & $0.3949 \mathrm{E}-08$ & $0.2440 \mathrm{E}-08$ & $0.1604 \mathrm{E}-08$ & $0.1200 \mathrm{E}-08$ & $0.9128 \mathrm{E}-09$ \\
\hline 2.7 & $0.3901 \mathrm{E}-08$ & $0.2397 \mathrm{E}-08$ & $0.1566 \mathrm{E}-08$ & $0.1165 \mathrm{E}-08$ & $0.8825 \mathrm{E}-09$ \\
\hline 2.8 & $0.3850 \mathrm{E}-08$ & $0.2353 \mathrm{E}-08$ & $0.1528 \mathrm{E}-08$ & $0.1131 E-08$ & $0.8536 \mathrm{E}-09$ \\
\hline 2.9 & $0.3798 \mathrm{E}-08$ & $0.2310 \mathrm{E}-08$ & $0.1492 E-08$ & $0.1099 \mathrm{E}-08$ & $0.8261 \mathrm{E}-09$ \\
\hline 3.0 & $0.3743 E-08$ & $0.2266 \mathrm{E}-08$ & $0.1456 \mathrm{E}-08$ & $0.1068 E-08$ & $0.7999 \mathrm{E}-09$ \\
\hline 3.1 & $0.3687 \mathrm{E}-08$ & $0.2223 E-08$ & $0.1421 \mathrm{E}-08$ & $0.1038 \mathrm{E}-08$ & $0.7749 \mathrm{E}-09$ \\
\hline 3.2 & $0.3630 \mathrm{E}-08$ & $0.2180 \mathrm{E}-08$ & $0.1387 \mathrm{E}-08$ & $0.1009 \mathrm{E}-08$ & $0.7511 \mathrm{E}-09$ \\
\hline 3.3 & $0.3573 E-08$ & $0.2138 \mathrm{E}-08$ & $0.1354 \mathrm{E}-08$ & $0.9810 \mathrm{E}-09$ & $0.7284 E-09$ \\
\hline 3.4 & $0.3516 \mathrm{E}-08$ & $0.2096 \mathrm{E}-08$ & $0.1322 E-08$ & $0.9544 E-09$ & $0.7067 \mathrm{E}-09$ \\
\hline 3.5 & $0.3459 \mathrm{E}-08$ & $0.2055 \mathrm{E}-08$ & $0.1291 E-08$ & $0.9289 \mathrm{E}-09$ & $0.6860 \mathrm{E}-09$ \\
\hline 3.6 & $0.3402 E-08$ & $0.2014 \varepsilon-08$ & $0.1261 \mathrm{E}-08$ & $0.9043 E-09$ & $0.6663 \mathrm{E}-09$ \\
\hline 3.7 & $0.3345 \mathrm{E}-08$ & $0.1975 \mathrm{E}-08$ & $0.1232 E-08$ & $0.8807 E-09$ & $0.6474 E-09$ \\
\hline 3.8 & $0.3289 \mathrm{E}-08$ & $0.1936 \mathrm{E}-08$ & $0.1204 E-08$ & $0.8581 \mathrm{E}-09$ & $0.6293 \mathrm{E}-09$ \\
\hline 3.9 & $0.3233 \mathrm{E}-08$ & $0.1898 \mathrm{E}-08$ & $0.1177 \mathrm{E}$ & $0.8363 E-09$ & $0.6121 \mathrm{E}-09$ \\
\hline 4.0 & $0.3179 \mathrm{E}-08$ & $0.1861 \mathrm{E}-08$ & $0.1150 \mathrm{E}-08$ & $0.8153 E-09$ & $0.5955 \mathrm{E}-09$ \\
\hline 4.1 & $0.3125 E-08$ & $0.1825 \mathrm{E}-08$ & $0.1125 \mathrm{E}-08$ & $0.7951 E-09$ & $0.5797 \mathrm{E}-09$ \\
\hline 4.2 & $0.3072 E-08$ & $0.1790 \mathrm{E}-08$ & $0.1100 E-08$ & $0.7757 \mathrm{E}-09$ & $0.5645 \mathrm{E}-09$ \\
\hline 4.3 & $0.3020 \mathrm{E}-08$ & $0.1756 \mathrm{E}-08$ & $0.1076 \mathrm{E}-08$ & $0.7571 \mathrm{E}-09$ & $0.5500 \mathrm{E}-09$ \\
\hline 4.4 & $0.2968 \mathrm{E}-08$ & $0.1722 E-08$ & $0.1053 \mathrm{E}-08$ & $0.7391 \mathrm{E}-09$ & $0.5360 \mathrm{E}-09$ \\
\hline 4.5 & $0.2918 \mathrm{E}-08$ & $0.1689 \mathrm{E}-08$ & $0.1030 E-08$ & $0.7218 E-09$ & $0.5226 \mathrm{E}-09$ \\
\hline 4.6 & $0.2869 \mathrm{E}-08$ & $0.1658 \mathrm{E}-08$ & $0.1008 \mathrm{E}-08$ & $0.7051 E-09$ & $0.5098 \mathrm{E}-09$ \\
\hline 4.7 & $0.2821 \mathrm{E}-08$ & $0.1627 \mathrm{E}-08$ & $0.9874 \mathrm{E}-09$ & $0.6890 \mathrm{E}-09$ & $0.4974 \mathrm{E}-09$ \\
\hline 4.8 & $0.2773 \mathrm{E}-08$ & $0.1596 \mathrm{E}-08$ & $0.9670 \mathrm{E}-09$ & $0.6735 \mathrm{E}-09$ & $0.4855 \mathrm{E}-09$ \\
\hline 4.9 & $0.2727 \mathrm{E}-08$ & $0.1567 \mathrm{E}-08$ & $0.9472 E-09$ & $0.6585 \mathrm{E}-09$ & $0.4741 \mathrm{E}-09$ \\
\hline 5.0 & $0.2682 E-08$ & $0.1538 \mathrm{E}-08$ & $0.9281 \mathrm{E}-09$ & $0.6441 E-09$ & $0.4631 \mathrm{E}-09$ \\
\hline
\end{tabular}


Table 25 e- $\mathrm{N}_{2}$ vibrational excitation rate coefficients, $\mathrm{cm}^{3} / \mathrm{s}$ $\left(v_{j}=10, \mathrm{~J}=50\right)$

\begin{tabular}{|c|c|c|c|c|c|}
\hline$[, \mathrm{eV}$ & $v_{f}=9$ & 8 & 7 & 6 & 5 \\
\hline 0.1 & $0.4310 \mathrm{E}-10$ & $0.7040 \mathrm{E}-10$ & $0.1072 E-09$ & $0.1490 \mathrm{E}-09$ & $0.1705 \mathrm{E}-09$ \\
\hline 0.2 & $0.1663 \mathrm{E}-09$ & 0.2337 E- 09 & $0.3142 E-09$ & $0.4305 \mathrm{E}-09$ & $0.4704 \mathrm{E}-09$ \\
\hline 0.3 & $0.3545 \mathrm{E}-09$ & $.4284 \mathrm{E}-09$ & $0.5301 \varepsilon-09$ & $0.6781 \mathrm{E}-09$ & $0.7296 \mathrm{E}-09$ \\
\hline 0.4 & $0.6211 \mathrm{E}-09$ & $0.6653 \mathrm{E}-09$ & $0.7610 \mathrm{E}-09$ & $0.9080 \mathrm{E}-09$ & $0.9518 \mathrm{E}-09$ \\
\hline 0.5 & $0.9560 \mathrm{E}-09$ & $0.9327 \mathrm{E}-09$ & $0.9904 \mathrm{E}-09$ & $0.1110 \mathrm{E}-08$ & $0.1126 \mathrm{E}-08$ \\
\hline 0.6 & $0.1333 E-08$ & $0.1209 \mathrm{E}-08$ & $0.1201 \mathrm{E}-08$ & $0.1273 \mathrm{E}-08$ & $0.1251 \mathrm{E}-08$ \\
\hline 0.7 & $0.1722 E-08$ & $0.1473 \mathrm{E}-08$ & $0.1381 \mathrm{E}-08$ & $0.1397 \mathrm{E}-08$ & $0.1332 E-08$ \\
\hline 0.8 & $0.2098 \mathrm{E}-08$ & $0.1712 E-08$ & $0.1529 \mathrm{E}-08$ & $0.1484 \mathrm{E}-08$ & $0.1378 \mathrm{E}-08$ \\
\hline 0.9 & $0.2444 \mathrm{E}-08$ & $0.1919 \mathrm{E}-08$ & $0.1643 \mathrm{E}-08$ & $0.1541 E-08$ & $0.1397 \varepsilon-08$ \\
\hline 1.0 & $0.2752 \mathrm{E}-08$ & $0.2092 \varepsilon-08$ & $0.1728 \mathrm{E}-08$ & $0.1572 \mathrm{E}-08$ & $0.1397 \mathrm{E}-08$ \\
\hline 1.1 & $0.3017 \mathrm{E}-08$ & $0.2233 \mathrm{E}-08$ & $0.1788 \mathrm{E}-08$ & $0.1585 \mathrm{E}-08$ & $0.1382 E-08$ \\
\hline 1.2 & $0.3241 E-08$ & $0.2343 E-08$ & $0.1827 \mathrm{E}-08$ & $0.1583 E-08$ & $0.1358 \mathrm{E}-08$ \\
\hline 1.3 & $0.3425 E-08$ & $0.2427 \mathrm{E}-08$ & $0.1848 E-08$ & $0.1570 \mathrm{E}-08$ & $0.1328 \mathrm{E}-08$ \\
\hline 1.4 & $0.3574 \mathrm{E}-08$ & $0.2488 \varepsilon-08$ & $0.1856 \mathrm{E}-08$ & $0.1549 \mathrm{E}-08$ & $0.1294 \mathrm{E}-08$ \\
\hline 1.5 & $0.3691 \mathrm{E}-08$ & $0.2530 E-08$ & $0.1853 E-08$ & $0.1522 \varepsilon-08$ & $0.1257 \varepsilon-08$ \\
\hline 1.6 & $0.3779 \mathrm{E}-08$ & $0.2555 E-08$ & $0.1841 E-08$ & $0.1491 \mathrm{E}-08$ & $0.1219 \mathrm{E}-08$ \\
\hline 1.7 & $0.3844 \mathrm{E}-08$ & $0.2567 \mathrm{E}-08$ & $0.1823 \varepsilon-08$ & $0.1458 E-08$ & $0.1180 E-08$ \\
\hline 1.8 & $0.3887 \mathrm{E}-08$ & $0.2568 \mathrm{E}-08$ & $0.1800 \mathrm{E}-08$ & $0.1422 E-08$ & $0.1142 E-08$ \\
\hline 1.9 & $0.3913 \mathrm{E}-08$ & $0.2560 E-08$ & $0.1772 E-08$ & $0.1386 \mathrm{E}-08$ & $0.1104 E-08$ \\
\hline 2.0 & $0.3924 E-08$ & $0.2544 E-08$ & $0.1742 E-08$ & $0.1350 E-08$ & $0.1068 \mathrm{E}-08$ \\
\hline 2.1 & $0.3923 E-08$ & $0.2522 E-08$ & $0.1710 \mathrm{E}-08$ & $0.1313 E-08$ & $0.1032 E-08$ \\
\hline 2.2 & $0.3911 \mathrm{E}-08$ & $0.2495 E-08$ & $0.1677 \mathrm{E}-08$ & $0.1277 E-08$ & $0.9978 \mathrm{E}-09$ \\
\hline 2.3 & $0.3890 \mathrm{E}-08$ & $0.2465 E-08$ & $0.1642 E-08$ & $0.1241 \mathrm{E}-08$ & $0.9647 \mathrm{E}-09$ \\
\hline 2.4 & $0.3862 E-08$ & $0.2431 E-08$ & 0.1607 & $0.1207 \mathrm{E}-08$ & $0.9330 \varepsilon-09$ \\
\hline 2.5 & $0.3828 \mathrm{E}-08$ & $0.2396 \mathrm{E}-08$ & 0.1572 & $0.1173 E-08$ & $0.9026 \mathrm{E}-09$ \\
\hline 2.6 & $0.3789 \mathrm{E}-08$ & $0.2358 \mathrm{E}-08$ & $0.1538 \mathrm{E}-$ & $0.1140 \mathrm{E}-08$ & $0.8734 \mathrm{E}-09$ \\
\hline 2.7 & $0.3747 \mathrm{E}-08$ & OE-08 & $0.1503 E-08$ & $0.1108 \mathrm{E}-08$ & $0.8456 \mathrm{E}-09$ \\
\hline 2.8 & $0.3701 E-08$ & $0.2281 \mathrm{E}-08$ & $0.1469 \mathrm{E}-08$ & $0.1077 \mathrm{E}-08$ & $0.8189 \mathrm{E}-09$ \\
\hline 2.9 & $0.3653 \mathrm{E}-08$ & 0.2 & 0.143 & $0.1048 \mathrm{E}-08$ & $4 E-09$ \\
\hline 3.0 & $0.3603 \mathrm{E}-08$ & -08 & 0.1403 & $0.1019 E-08$ & $0.7691 \varepsilon-09$ \\
\hline 3.1 & $0.3551 \mathrm{E}-08$ & 0.2 & 0.1371 & $0.9914 \mathrm{E}-09$ & $0.7458 \mathrm{E}-09$ \\
\hline 3.2 & $0.3499 \mathrm{E}-08$ & 0.2 & 0.1 & $0.9648 \mathrm{E}-09$ & $0.7236 \mathrm{E}-09$ \\
\hline 3.3 & $0.3446 E-08$ & $0.2081 \mathrm{E}-08$ & $0.1309 \mathrm{E}-08$ & $0.9391 \mathrm{E}-09$ & $0.70248-09$ \\
\hline 3.4 & & & 0.127 & $0.9144 \mathrm{E}-09$ & $0.6821 \mathrm{E}-09$ \\
\hline 3.5 & $B E-08$ & 0.2 & $0.125 \mathrm{C}$ & $0.8906 \mathrm{E}-09$ & $0.6626 \mathrm{E}-09$ \\
\hline 3.6 & $0.3285 \mathrm{E}-08$ & $0.1966 \mathrm{~B}$ & & $0.8678 \mathrm{E}-$ & \\
\hline 3.7 & $0.3232 E-08$ & 0.19 & 0.11 & $0.8457 \mathrm{E}-$ & $E-09$ \\
\hline 3.8 & $0.3179 \mathrm{E}-08$ & $0.1892 \mathrm{E}-08$ & $0.1168 \varepsilon-08$ & $0.8245 E-09$ & $0.6092 E-09$ \\
\hline 3.9 & 0.212 & & & $0.8041 \mathrm{E}-09$ & $0.5929 \mathrm{E}-09$ \\
\hline 4.0 & $0.3 c$ & 0. & 0.111 & $0.7844 \mathrm{E}-09$ & 0.577 \\
\hline 4.1 & $0.3024 E-08$ & $0.1787 \mathrm{E}-08$ & $0.1093 \varepsilon-08$ & $0.7655 E-09$ & $0.5622 E-09$ \\
\hline 4.2 & $0.2974 E-08$ & $0.1753 E-08$ & $0.1070 E-08$ & $0.7472 E-09$ & $0.5478 \mathrm{E}-09$ \\
\hline 4.3 & $0.2924 E-08$ & $0.1720 \mathrm{E}-08$ & $0.1047 \mathrm{E}-08$ & $0.7297 \varepsilon-09$ & $0.5340 \mathrm{E}-09$ \\
\hline 4.4 & $0.2876 \mathrm{E}-08$ & & & & $0.5207 \mathrm{E}-09$ \\
\hline 4.5 & $0.2828 \mathrm{E}-08$ & $0.1657 \mathrm{E}-08$ & $0.1004 \mathrm{E}$ & $0.6964 E-09$ & $0.50798-09$ \\
\hline 4.6 & $0.2781 \mathrm{E}-08$ & $0.1627 \mathrm{E}-08$ & $0.9829 \mathrm{E}-09$ & $0.6806 \mathrm{E}-09$ & $0.4957 \varepsilon-09$ \\
\hline 4.7 & $0.2735 \mathrm{E}-08$ & $0.1597 \mathrm{E}-08$ & $0.9629 \mathrm{E}-09$ & $0.6654 \mathrm{E}-09$ & $0.4839 \mathrm{E}-09$ \\
\hline 4.8 & $0.2690 \mathrm{E}-08$ & $0.1568 \mathrm{E}-08$ & $0.9434 \mathrm{E}-$ & $0.6507 \mathrm{E}-09$ & $0.4725 \mathrm{E}-09$ \\
\hline 4. & $0.2646 \mathrm{E}-\mathrm{C}$ & $0.1540 \mathrm{E}-$ & $0.9245 E$ & & $0.4616 \mathrm{E}-09$ \\
\hline$=0$ & $0.2602 E-08$ & $0.1512 F_{-}$ & $0.9062 \mathrm{E}$ & $0.6228 \mathrm{E}-09$ & $0.4511 \mathrm{E}-09$ \\
\hline
\end{tabular}


Table 26 e-N 2 vibrational excitation rate coefficients, $\mathrm{cm}^{3} / \mathrm{s}$ $\left(v_{1}=11, J=50\right)$

\begin{tabular}{|c|c|c|c|c|c|}
\hline $\mathrm{T}, \mathrm{eV}$ & $v_{f}=10$ & 9 & 8 & 7 & 6 \\
\hline 0.1 & $0.3697 \mathrm{E}-10$ & $0.6105 \varepsilon-10$ & $0.1017 \mathrm{E}-09$ & $0.1333 \mathrm{E}-09$ & $0.1762 \mathrm{E}-09$ \\
\hline 0.2 & $0.1357 \mathrm{E}-09$ & $0.1801 \mathrm{E}-09$ & $0.2632 \mathrm{E}-09$ & $0.3371 \mathrm{E}-09$ & $0.4112 E-09$ \\
\hline 0.3 & $0.3027 \varepsilon-09$ & $0.3452 E-09$ & $0.4484 E-09$ & $0.5429 E-09$ & $0.6311 \mathrm{E}-09$ \\
\hline 0.4 & $0.5469 \mathrm{E}-09$ & $0.5607 \mathrm{E}-09$ & $0.6566 \mathrm{E}-09$ & $0.7515 E-09$ & $0.8326 \mathrm{E}-09$ \\
\hline 0.5 & $0.8582 \mathrm{E}-09$ & $0.8112 E-09$ & $0.8693 \mathrm{E}-09$ & $0.9430 \mathrm{E}-09$ & $0.9984 \mathrm{E}-09$ \\
\hline 0.6 & $0.1212 E-08$ & $0.1074 E-08$ & $0.1069 \mathrm{E}-08$ & $0.1104 \mathrm{E}-08$ & $0.1122 E-08$ \\
\hline 0.7 & $0.1581 E-08$ & $0.1330 \mathrm{E}-08$ & $0.1243 \mathrm{E}-08$ & $0.1230 E-08$ & $0.1207 E-08$ \\
\hline 0.8 & $0.1940 \mathrm{E}-08$ & $0.1564 \mathrm{E}-08$ & $0.1389 \mathrm{E}-08$ & $0.1322 E-08$ & $0.1259 \mathrm{E}-08$ \\
\hline 0.9 & $0.2273 E-08$ & $0.1770 \mathrm{E}-08$ & $0.1505 E-$ & $0.1386 \mathrm{E}-08$ & $0.1286 \mathrm{E}-08$ \\
\hline 1.0 & $0.2571 \mathrm{E}-08$ & $0.1944 \mathrm{E}-08$ & $0.1593 \mathrm{E}-08$ & $0.1426 \mathrm{E}-08$ & $0.1293 E-08$ \\
\hline 1.1 & $0.2830 \mathrm{E}-08$ & 0.2087 E- 08 & $0.1658 \mathrm{E}-08$ & $0.1448 E-08$ & $0.1287 E-08$ \\
\hline 1.2 & $0.3050 \mathrm{E}-08$ & $0.2202 E-08$ & $0.1702 E-08$ & $0.1454 \mathrm{E}$ & $0.1270 E-08$ \\
\hline 1.3 & $0.3232 E-08$ & $0.2291 \varepsilon-08$ & $0.1730 \mathrm{E}-08$ & $0.1449 \mathrm{E}-08$ & $0.1247 \mathrm{E}-08$ \\
\hline 1.4 & $0.3381 E-08$ & $0.2358 \mathrm{E}-08$ & $0.1744 \mathrm{E}-08$ & $0.1436 \mathrm{E}-08$ & $0.1219 E-08$ \\
\hline 1.5 & $0.3499 E-08$ & $0.2405 E-08$ & $0.1747 \varepsilon-08$ & $0.1417 \varepsilon-08$ & $0.1188 \mathrm{E}-08$ \\
\hline 1.6 & $0.3590 \mathrm{E}-08$ & $0.2436 \mathrm{E}-08$ & $0.1742 \mathrm{E}-08$ & $0.1393 E-08$ & $0.1155 \mathrm{E}-08$ \\
\hline 1.7 & $0.3657 \varepsilon-08$ & $0.2454 \mathrm{E}-08$ & $0.1729 \mathrm{E}-08$ & 0.1365 & $0.1122 E-08$ \\
\hline 1.8 & $0.3704 E-08$ & $0.2461 E-08$ & $0.1711 \mathrm{E}-08$ & $0.1336 \mathrm{E}-08$ & $0.1088 E-08$ \\
\hline 1.9 & $0.3735 \mathrm{E}$ & $0.2458 \mathrm{E}-08$ & $0.1689 \mathrm{E}-08$ & $0.1305 E-08$ & $0.1054 \mathrm{E}-08$ \\
\hline 2.0 & $0.3750 \mathrm{E}-$ & $0.2447 \mathrm{E}$ & 0.1664 & 0.1273 & $0.1021 \mathrm{E}-08$ \\
\hline 2.1 & $0.3753 E-08$ & $0.2430 \mathrm{E}-08$ & $0.1636 \mathrm{E}-08$ & 0.124 & $0.9887 \mathrm{E}-09$ \\
\hline 2.2 & $0.3745 \mathrm{E}-08$ & $0.2408 \mathrm{E}-08$ & $0.1607 \varepsilon-08$ & $0.1210 E-08$ & $0.9573 \mathrm{E}-09$ \\
\hline 2.3 & $0.3729 \mathrm{E}$ & 0.2 & 0.1576 & $0.1178 E-08$ & $0.9269 \mathrm{E}-09$ \\
\hline 2.4 & $0.3706 \mathrm{E}-08$ & $0.2353 \mathrm{E}-08$ & 0.15 & 0.11 & $0.8977 \mathrm{E}-09$ \\
\hline 2.5 & $0.3676 \mathrm{E}-08$ & $0.2322 \mathrm{E}-$ & $0.1514 E-08$ & $6 E-08$ & $0.8695 \mathrm{E}-09$ \\
\hline 2.6 & $0.3642 E-08$ & $0.2288 \mathrm{E}-08$ & $0.1482 E-08$ & $0.1086 \mathrm{E}-08$ & $0.8424 \mathrm{E}-09$ \\
\hline 2.7 & $0.3604 \mathrm{E}-08$ & $0.2253 E-08$ & $0.1451 \mathrm{E}-08$ & $0.1057 \mathrm{E}-08$ & $0.8164 E-09$ \\
\hline 2.8 & $0.3562 E-08$ & $0.2217 \mathrm{E}-08$ & $0.1419 E-08$ & $0.1029 E-08$ & $0.7915 \mathrm{E}-09$ \\
\hline 2.9 & $0.3518 \mathrm{E}-08$ & $0.2181 E-08$ & 0.1 & 0.10 & $0.7676 \mathrm{E}-09$ \\
\hline 3.0 & $0.3472 E-08$ & $0.2144 \mathrm{E}-08$ & $0.1358 \mathrm{E}$ & $\varepsilon-09$ & $0.7447 E-09$ \\
\hline 3.1 & $0.3424 E-08$ & 0.2 & 0.1 & 0.9 & 0.722 \\
\hline 3.2 & 0.3 & 0.2 & -08 & $\varepsilon-09$ & $0.7019 \mathrm{E}-09$ \\
\hline 3.3 & $0.3326 \mathrm{E}-08$ & $0.2032 E-08$ & 0.1 & 09 & $0.6818 \mathrm{E}-09$ \\
\hline 3.4 & $16=00$ & $6 E-08$ & 0.12 & 0.8 & 0.66 \\
\hline 3.5 & 8 & $0.1959 \mathrm{E}-08$ & -08 & $0.8564 E-09$ & $0.6441 E-09$ \\
\hline 3.6 & 0.3 & $0.1923 \mathrm{E}-$ & 0.1 & & \\
\hline 3.7 & $5 E-08$ & $0.1888 E-08$ & 0.11 & 0.81 & $0.6095 \mathrm{E}-09$ \\
\hline 3.8 & $5 E-08$ & $0.1853 \mathrm{E}-08$ & $0.1138 \mathrm{E}-08$ & $0.7944 E-09$ & $0.5932 E-09$ \\
\hline 3.9 & 0.3 & & & & $0.5776 \mathrm{E}-09$ \\
\hline 4.0 & 0.2 & $0.1785 E-0$ & 0.10 & $0.7567 E-09$ & $0.5627 \mathrm{E}-09$ \\
\hline 4.1 & $0.2928 \mathrm{E}-08$ & $0.1753 E-08$ & $0.1067 \mathrm{E}-08$ & $9 \mathrm{E}-09$ & $0.5483 \mathrm{E}-09$ \\
\hline 4.2 & $0.2880 \mathrm{E}-08$ & $0.1720 \mathrm{E}-08$ & $0.1044 \mathrm{E}-08$ & 0.72 & $0.5345 E-09$ \\
\hline 4.3 & $0.2833 E-$ & $0.1689 E-08$ & 0.102 & $0.7050 \mathrm{E}-09$ & $0.5213 \mathrm{E}-09$ \\
\hline 4.4 & $0.2787 E-08$ & $0.1658 E-08$ & $0.1002 E-08$ & $0.6890 E-09$ & $0.5085 \mathrm{E}-09$ \\
\hline 4.5 & $0.2741 \mathrm{E}-08$ & $0.1628 \mathrm{E}-08$ & $0.9813 \mathrm{E}-09$ & $0.6735 \mathrm{E}-09$ & $0.4963 \mathrm{E}-09$ \\
\hline 4.6 & $0.2697 \mathrm{E}-08$ & $0.1599 \mathrm{E}-08$ & $0.9615 \mathrm{E}-09$ & $0.6586 \mathrm{E}-09$ & $0.4845 E-09$ \\
\hline 4.7 & $0.2653 \mathrm{E}-$ & $0.1570 \mathrm{E}-08$ & $0.9423 E-09$ & $0.6441 E-09$ & $0.4732 \mathrm{E}-09$ \\
\hline 4.8 & $0.2610 \mathrm{E}-$ & $0.1542 E-08$ & $0.9236 \mathrm{E}-09$ & $0.6302 E-09$ & $0.4622 \mathrm{E}-09$ \\
\hline 4.9 & $0.2567 \mathrm{E}-\mathrm{C}$ & $0.1515 \mathrm{E}-$ & $0.9055 \mathrm{E}-09$ & $0.6167 \mathrm{E}-09$ & $0.4517 \mathrm{E}-09$ \\
\hline 5.0 & $0.2526 \mathrm{E}-08$ & $0.1488 \mathrm{E}-08$ & $0.8879 \mathrm{E}-09$ & $0.6036 \varepsilon-09$ & $0.4416 \mathrm{E}-09$ \\
\hline
\end{tabular}


Table 27 e- $\mathrm{N}_{2}$ vibrational excitation rate coefficients, $\mathrm{cm}^{3} / \mathrm{s}$ $\left(v_{j}=12, J=50\right)$

\begin{tabular}{|c|c|c|c|c|c|}
\hline $\mathrm{T}, \mathrm{eV}$ & $v_{f}=11$ & 10 & 9 & 8 & 7 \\
\hline 0.1 & $0.2873 E-10$ & $0.4922 E-10$ & $0.8488 \mathrm{E}-10$ & $0.1097 \mathrm{E}-09$ & $0.1574 E-09$ \\
\hline 0.2 & $0.1088 \mathrm{E}-09$ & $0.1424 E-09$ & $0.2108 \mathrm{E}-09$ & $0.2627 E-09$ & \\
\hline 0.3 & $0.2588 \mathrm{E}-09$ & $0.2909 \mathrm{E}-09$ & $0.3738 \mathrm{E}-09$ & $0.4459 \mathrm{E}-09$ & $0.5332 E-09$ \\
\hline 0.4 & $0.4839 \mathrm{E}-09$ & $0.4911 \mathrm{E}-09$ & $0.5645 \mathrm{E}-09$ & $0.6408 \mathrm{E}-09$ & $0.7207 \mathrm{E}-09$ \\
\hline 0.5 & $0.7747 \mathrm{E}-09$ & $0.7275 \mathrm{E}-09$ & $0.7637 \varepsilon-09$ & $0.8237 \mathrm{E}-09$ & $0.8807 E-09$ \\
\hline 0.6 & $0.1109 \mathrm{E}-08$ & $0.9788 \mathrm{E}-09$ & $0.9540 \mathrm{E}-09$ & $0.9806 \mathrm{E}-09$ & $0.1004 E-08$ \\
\hline 0.7 & $0.1459 \mathrm{E}-08$ & $0.1226 \mathrm{E}-08$ & $0.1124 \mathrm{E}-08$ & $0.1106 \mathrm{E}-08$ & 0.109 \\
\hline 0.8 & $0.1802 E-08$ & $0.1454 \mathrm{E}-08$ & $0.1268 \mathrm{E}-08$ & $0.1201 \mathrm{E}-08$ & $0.1150 E-08$ \\
\hline 0.9 & $0.2123 \mathrm{E}-08$ & 0.1657 & $0.1385 E-$ & $0.1269 \mathrm{E}-08$ & $0.1184 E-08$ \\
\hline 1.0 & $0.2411 \mathrm{E}-08$ & $0.1830 \mathrm{E}-08$ & $0.1476 \mathrm{E}-08$ & 0.1315 & $0.1198 \mathrm{E}-08$ \\
\hline 1.1 & $0.2664 E-08$ & $0.1975 \mathrm{E}-08$ & $0.1545 E-08$ & $0.1342 \varepsilon-08$ & $0.1199 \mathrm{E}-08$ \\
\hline 1.2 & $0.2879 \mathrm{E}$ & $0.2092 \mathrm{E}-08$ & $0.1594 \mathrm{E}-08$ & $0.1354 \mathrm{E}-08$ & $0.1189 \mathrm{E}-08$ \\
\hline 1.3 & $0.3060 E-08$ & 0.218 & $0.1627 \mathrm{E}-08$ & 0.1355 & $0.1172 E-08$ \\
\hline 1.4 & $0.3207 \varepsilon-08$ & 0.225 & $0.1646 \mathrm{E}-08$ & & $9 E-08$ \\
\hline 1.5 & $0.3326 \mathrm{E}-08$ & $0.2306 \mathrm{E}-$ & $0.1655 \mathrm{E}-$ & $0.1333 \mathrm{E}$ & $E-08$ \\
\hline 1.6 & $0.3418 \mathrm{E}-08$ & 0.23 & $0.1654 \mathrm{E}-08$ & $0.1315 \mathrm{E}-08$ & $0.1096 \mathrm{E}-08$ \\
\hline 1.7 & $0.3488 E-08$ & 0.2364 & $0.1647 \mathrm{E}-08$ & $0.1292 \mathrm{E}-08$ & $0.1067 \varepsilon-08$ \\
\hline 1.8 & $0.3538 \mathrm{E}-08$ & $0.2375 E-08$ & $0.1633 \mathrm{E}-08$ & $0.1267 \mathrm{E}-08$ & $0.1037 E-08$ \\
\hline 1.9 & $0.3571 \mathrm{E}-08$ & 0.23 & $0.1616 \mathrm{E}-08$ & 0.124 & $E-08$ \\
\hline 2.0 & $0.3590 \mathrm{E}-08$ & $0.2370 E-08$ & $0.1595 \mathrm{E}-$ & 0.121 & $0.9768 \mathrm{E}-09$ \\
\hline 2.1 & 0.3597 & 0.235 & $0.1571 \mathrm{E}$ & 0.118 & $0.9475 E-09$ \\
\hline 2.2 & $0.3593 \mathrm{E}-($ & $0.2339 \mathrm{E}-08$ & $0.1546 E-08$ & $0.1156 \mathrm{E}-08$ & $0.9189 \mathrm{E}-09$ \\
\hline 2.3 & $0.3581 \varepsilon-08$ & $0.2317 E-08$ & $0.1519 \mathrm{E}-08$ & $0.1127 E-08$ & $0.8910 \mathrm{E}-09$ \\
\hline 2.4 & $0.3561 \mathrm{E}$ & 0.22 & $1 E-08$ & 0.109 & $0.8640 \mathrm{E}-09$ \\
\hline 2.5 & $0.3535 E$ & $0.2263 E-08$ & $0.1463 E-$ & $0.1071 \mathrm{E}-08$ & $0.8379 E-09$ \\
\hline 2.6 & $0.3505 \mathrm{E}-08$ & 0.22 & 0.1 & $0.1044 E-08$ & $0.8127 E-09$ \\
\hline 2.7 & $0.3470 \mathrm{E}-08$ & $0.2200 \mathrm{E}-08$ & $0.1405 E-08$ & 0.1017 E- 08 & $0.7885 \mathrm{E}-09$ \\
\hline 2.8 & 0.3432 & 0.21 & $0.1376 \mathrm{E}-$ & 0.990 & $0.7651 \mathrm{E}-09$ \\
\hline 2.9 & $0.3392 E-08$ & 0.21 & $0.1347 \mathrm{E}-08$ & $0.9655 \mathrm{E}-09$ & $0.7427 E-09$ \\
\hline 3.0 & 0.3349 & $0.2098 \mathrm{E}-08$ & $0.1319 E-08$ & $0.9408 \mathrm{E}-09$ & $0.7212 E-09$ \\
\hline 3.1 & $0.3305 E-08$ & 0.2 & 0.1 & 0.9 & $E-09$ \\
\hline 3.2 & $0.3259 \mathrm{E}-0$ & 0.2028 & 0.12 & 0.893 & $0.6808 \mathrm{E}-09$ \\
\hline 3.3 & $0.3213 \mathrm{E}$ & 0.1 & 0.12 & 0.87 & $3 E-09$ \\
\hline 3.4 & $0.3166 \mathrm{E}-\mathrm{C}$ & 0.15 & 0.12 & $E-09$ & 0.64 \\
\hline 3.5 & $0.3118 \mathrm{E}-$ & 0.19 & $0.1185 E-08$ & $0.8290 \mathrm{E}-09$ & $0.6260 \mathrm{E}-09$ \\
\hline 3.6 & 0.30 & 0.1 & 0.1 & $0.8088 \mathrm{E}-09$ & $0.6092 E-09$ \\
\hline 3.7 & $0.3023 \mathrm{E}-08$ & $0.1856 \mathrm{E}-08$ & $0.1136 \mathrm{E}-08$ & $0.7893 \mathrm{E}-09$ & $0.5931 \mathrm{E}-09$ \\
\hline 3.8 & 0.297 & & & & $0.5776 \mathrm{E}-09$ \\
\hline 3.9 & 0.29 & 0.1 & 0.1 & 0.7 & $0.5627 \mathrm{E}-09$ \\
\hline 4.0 & 0.2883 & 0.175 & $0.1066 \mathrm{E}-08$ & $0.7347 \mathrm{E}-09$ & $0.5484 \mathrm{E}-09$ \\
\hline 4.1 & $0.2837 \mathrm{E}-0$ & 0.17 & 0.10 & E-09 & 0.5347 E-09 \\
\hline 4.2 & $0.2791 \mathrm{E}-08$ & .16 & 0.1 & $3 E-09$ & $0.5215 \mathrm{E}-09$ \\
\hline 4.3 & 0.27 & & & $0.6855 E-09$ & $0.5087 E-09$ \\
\hline 4.4 & 0.27 & 0.16 & $0.9818 E-$ & $0.6702 E-09$ & $0.4965 \mathrm{E}-09$ \\
\hline 4.5 & $0.2659 \mathrm{E}-08$ & $0.1606 \mathrm{E}-08$ & $0.9622 E-09$ & $0.6554 E-09$ & $0.4847 E-09$ \\
\hline 4.6 & $0.2616 E-08$ & & $0.9432 E-09$ & $0.6411 \mathrm{E}-09$ & $0.4734 \mathrm{E}-09$ \\
\hline 4.7 & 0.25 & 0.154 & & $0.6273 E-09$ & $0.4625 \mathrm{E}-09$ \\
\hline 4.8 & & 0.15 & 0. & 0.6 & $0.4520 \mathrm{E}-09$ \\
\hline 4.9 & $0.2492 E-C$ & $0.1496 \mathrm{E}-0$ & & & $0.4418 \mathrm{E}-09$ \\
\hline 5.0 & $0.2452 E-08$ & $0.1470 \mathrm{E}-08$ & $0.8723 E-09$ & $0.5885 E-09$ & $0.4320 \mathrm{E}-09$ \\
\hline
\end{tabular}




\section{References}

${ }^{1}$ Menees, G. P., "Trajectory Analysis of Radiative Heating for Planetary Missions with Aerobraking of Spacecraft," Journal of Spacecraft and Rockets, Vol. 22, Jan.-Feb. 1985, pp. 37-45.

${ }^{2}$ Park, C., "Problems of Rate Chemistry in the Flight Regimes of Aeroassisted Orbital Transfer Vehicles," AIAA Paper 84-1730, June 1984.

3Park, C., "Radiation Enhancement by Nonequilibrium in Earth's Atmosphere," Journal of Spacecraft and Rockets, Vol. 22, Jan.-Feb. 1985, pp. $27-36$.

${ }^{4}$ Cooper, D. M., Jaffe, R. L., and Arnold, J. 0., "Computational Chemistry and Aeroassisted Orbital Transfer Vehicles," Journal of Spacecraft and Rockets, Vol. 22, Jan.-Feb. 1985, pp. 60-67.

${ }^{5}$ Lee, J. H., "Electron-Impact Vibrational Excitation Rates in the Flow-Field of Aeroassisted Orbital Transfer Vehicles," AIAA Paper 85-1035, June 1985.

${ }^{6}$ Schulz, G. J., "Resonances in Electron Impact on Diatomic Molecules," Review of Modern Physics, Vol. 45, 1973, pp. 423-486.

${ }^{7}$ Schulz, G. J., "A Review of Vibrational Excitation of Molecules by Electron Impact at Low Energies," Principles of Laser Plasmas, edited by G. Bekefi, John Wiley \& Sons, New York, 1976, pp. 33-69.

${ }^{8}$ Ehrhardt, H. and Willmann, K., "Die Winkelabhängigkeit der Resonanzstreuung Niederenergetischer Elektronen an $\mathrm{N}_{2}$," Zeitschrift für Physik, Vol. 204, 1967, pp. 462-473.

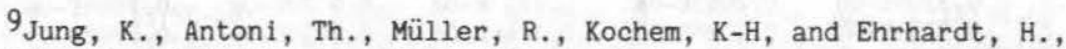
"Rotational Excitation of $\mathrm{N}_{2}, \mathrm{CO}$, and $\mathrm{H}_{2} \mathrm{O}$ by Low-Energy Electron Collisions," Journal of Physics B: Atomic and Molecular Physics, Vol. 15, 1982, pp. 3535-3555.

10 Wong, S. F., Michejda, J. A., and Stamatovic, A., unpublished data.

${ }^{11}$ Birtwistle, D. T. and Herzenberg, A., "Vibrational Excitation of $\mathrm{N}_{2}$ by Resonance Scattering of Electrons," Journal of Physics B: Atomic and Molecular Physics, Vol. 4, 1971, pp. 53-70.

${ }^{12}$ (a) Schneider, B. I., Le Dourneuf, M., and Lan, V. K., "Resonant Vibrational Excitation of $\mathrm{N}_{2}$ by Low-Energy Electron Impact," Physical Review Letters, Vol. 43, 1979, pp. 1926-1929. (b) Le Dourneuf, M., Lan, V. K., and Schneider, B. I., "Recent Developments of the Frame Transformation Theory of Electron-Molecule Processes," in Electron-Atom and Electron-Molecule Collisions, edited by J. Hinze, Plenum Press, New York, 1983, pp. 135-136. 
${ }^{13}$ Dubé, L. and Herzenberg, A., "Absolute Cross Sections from the 'Boomerang Model' for Resonant Electron-Molecule Scattering," Physical Review, Vol. A20, 1979, pp. 194-213.

${ }^{14}$ (a) N. Chandra and A. Temkin, "Hybrid Theory and Calculation of e-N 2 Scattering," Physical Review, Vol. A13, 1976, pp. 188-203;

(b) N. Chandra and A. Temkin, "Hybrid Theory Calculation of Simultaneous Vibration-Rotation Excitation in e-N 2 Scattering," Physical Review, Vol. A14, 1976, pp. 507-511; (c) N. Chandra and A. Temkin, "Tabulation of Hybrid Theory Calculated e-N 2 Vibrational and Rotational Cross Sections," NASA Technical Note TN D 8347, 1976.

${ }^{15}$ Hazi, A. U., Rescigno, T. N., and Kurilla, M., "Cross Sections for Resonant Vibrational Excitation of $\mathrm{N}_{2}$ by Electron Impact," Physical Review, Vol. A23, 1981, pp. 1089-1098.

${ }^{16}$ Hazi, A. U., "Molecular Resonance Phenomena," Electron-Atom and Electron-Molecule Collisions, edited by J. Hinze, Plenum Press, New York, 1983, pp. 103-119.

${ }^{17}$ Berman, M., Estrada, H., Cederbaum, L. S., and Domcke, W., "Nuclear Dynamics in Resonant Electron-Molecule Scattering Beyond the Local Approximation: The $2.3 \mathrm{eV}$ Shape Resonance in $\mathrm{N}_{2}$," Physical Review, Vol. A28, 1983, pp. 1363-1381.

$18_{\mathrm{Ali}}$, A. W., "Excitation and Ionization Cross Sections for Electron Beam and Microwave Energy Deposition in Air," Naval Research Laboratory, Washington, D.C., NRL Memorandum Rept. 4598, Aug . 1981.

${ }^{19}$ Slinker, S. and Ali, A. W., "Electron Excitation and Ionization Rate Coefficients for $\mathrm{N}_{2}, \mathrm{O}_{2}, \mathrm{NO}, \mathrm{N}$, and $\mathrm{O}$, " Naval Research Laboratory, Washington, D.C., NRL Memorandum Rept. 4756, Feb. 1982.

${ }^{20}$ Takatsuka, K. and McKoy, V., "Theory of Electronically Inelastic Scattering of Electrons by Molecules," Physical Review, Vol. A30, 1984 , pp. $1734-1740$.

${ }^{21}$ Huo, W. M., Gibson, T. L., Marco, M. A. P., and McKoy, V., Physical Review, Vol. A (to be submitted).

22 Huo, W. M., Marco, M. A. P., Gibson, T. L., and McKoy, V., Physical Review, Vol. A (to be submitted).

${ }^{23}$ Langhoff, S. R., Bauschlicher, C. W., Jr., and Chong, D. P., "Theoretical Study of the Effects of Vibrational-Rotational Interactions in the Raman Spectrum of $\mathrm{N}_{2}$," Journal of Chemical Physics, Vol. 78,1983 , pp. 5287-5292. 
${ }^{24}$ Gibson, T. L., Lima, M. A. P., Takatsuka, K., and McKoy, V., "Ab initio Inclusion of Polarization Effects in the Schwinger Multichannel Formulation: Application to Elastic e- $\mathrm{H}_{2}$ Scattering," Physical Review, Vol. A30, 1984, pp. 3005-3011.

${ }^{25}$ Schneider, B. I. and Collins, L. A., "Comparative Study of LowEnergy ${ }^{2} \Sigma_{g}^{+}$and ${ }^{2} \pi_{g}$ Scattering in Molecular Nitrogen," Physical Review, Vol. A30, 1984, pp. 95-99.

${ }^{26}$ Feshbach, H., "A Unified Theory of Nuclear Reactions, II," Annals of Physics, Vol. 19, 1962, pp. 287-313.

27 Loftus, A. and Krupenie, P. H., "The Spectrum of Molecular Nitrogen," Journal of Physical and Chemical Reference Data, Vol. 6, 1977, pp. 113-307.

28 Bauschlicher, C. W., Jr., "A Comment on 'The Effect of Bond Functions on Molecular Dissociation Energies, by Wright and Buenker'," Chemical Physics Letters (in press).

${ }^{29}$ Gibson, T. L. and Morrison, M. A., Physical Review, Vol. A (to be published).

30 Wigner, E. P., "On the Behavior of Cross Sections Near Thresholds," Physical Review, Vol. 73, 1948, pp. 1002-1009.

${ }^{31}$ Schulz, G. J., "Vibrational Excitation of $\mathrm{N}_{2}, \mathrm{CO}$, and $\mathrm{H}_{2}$ by Electron Impact," Physical Review, Vol. 135, 1964, pp. A988-A1004. 